Um estudo sobre a equação de Hénon 

SERVIÇO DE PÓS-GRADUAÇÃO DO ICMC-USP

Data de Depósito:

Assinatura:

\title{
Um estudo sobre a equação de Hénon
}

\author{
Maribel Rosa Bravo Quispe
}

Orientador: Prof. Dr. Ederson Moreira dos Santos

\begin{abstract}
Dissertação apresentada ao Instituto de Ciências Matemáticas e de Computação - ICMC-USP, como parte dos requisitos para obtenção do título de Mestre em Ciências - Matemática . VERSÃO REVISADA
\end{abstract}


Ficha catalográfica elaborada pela Biblioteca Prof. Achille Bassi e Seção Técnica de Informática, ICMC/USP, com os dados fornecidos pelo(a) autor(a)

Bravo Quispe, Maribel Rosa
Um estudo sobre a equação de Hénon / Maribel Rosa
Bravo Quispe; orientador Ederson Moreira dos Santos.
-- São Carlos, 2013.
79 p.
Dissertação (Mestrado - Programa de Pós-Graduação en
Matemática) -- Instituto de Ciências Matemáticas e
de Computação, Universidade de São Paulo, 2013.
1. Equação de Hénon. 2. Solução Ground State. 3.
Quebra de simetria. 4. Polarizac̃o e simetria de
Schwarz folheada. 5. Multiplicidade de soluções
positivas. I. Moreira dos Santos, Ederson, orient.
II. Título.


A Matemática é a chave de ouro, com que podemos abrir todas as ciências.

Victor Duruy. 



\section{Agradecimentos}

A Deus por tudo, que me fortaleceu e me deu coragem para seguir mesmo em tempos de aflição e fraqueza, não tenho palavras para expressar o quão grata sou a Deus. É Ele quem me concede, por sua infinita misericórdia, todas as coisas que tenho conquistado até este dia. Por isso a Ele seja dada toda honra, glória e louvor por que é digno!

Aos meus pais Juan Bravo e Nazaria Quispe, pela paciência que tiveram comigo em minha infância, por todo apoio, amor e motivação em minha vida. Amo muito vocês!

Aos meus irmãos Carlos, Alberto e Jesus, pela amizade incondicional, pelos conselhos, pela torcida, pelo companheirismo e carinho durante estes anos. Desejo que vocês continuem conquistando grandes coisas, boas coisas, que Deus seja o centro da vida de vocês, pois, quando se coloca Deus no controle Ele se encarrega do resto.

Ao meu orientador Ederson Moreira dos Santos, por aceitar ser meu orientador, por ter acreditado em mim, por estar sempre disposto a me ajudar desde que cheguei nesta universidade, pelos conselhos, dedicação e amizade durante todo o período em que me orientou. Agradeço também pela paciência que demonstrou em vários momentos, por todo conhecimento adquirido e por fazer-me estudar muito, tanto assim que cheguei gostar de análise funcional e querer continuar meu estudo nas EDP elípticas. Muito obrigada por tudo e desejo tudo de bom para você.

A meu namorado Misael, pelo amor, pela torcida, pela paciência nos momentos em que estive distante, por se preocupar comigo, por me apoiar em tudo e por suas orações. EU TE AMO!

Aos professores do Programa de Pós-Graduação do ICMC e aos demais professores que participaram da minha vida universitária, os quais considero fundamentais para minha formação. Agradeço especialmente ao professor Agripino Garcia Armas, que foi aquele que mais me incentivou para fazer um mestrado.

Aos meus amigos do ICMC. Dada a quantidade de pessoas que eu poderia listar 
neste parágrafo, omitirei os nomes para evitar prováveis esquecimentos. No entanto, posso dizer que eles não só participaram de momentos de descontração, mas também tiveram presença em momentos complicados e decisivos. Desejo toda a felicidade do mundo para cada um de vocês.

Ao CNPq - Conselho Nacional de Desenvolvimento Científico e Tecnológico, pelo apoio financeiro e à coordenação da Pós Graduação em Matemática da USP. 
Aos meus queridos pais, Juan e Nazaria. 


\section{Resumo}

Este trabalho apresenta um estudo quantitativo e qualitativo de soluções positivas para o problema de Dirichlet para a equação de Hénon

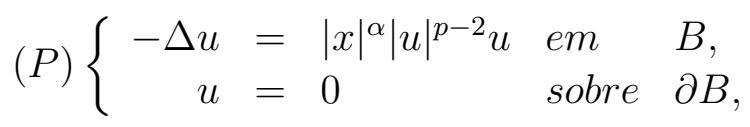

onde $B$ é a bola unitária aberta de $\mathbb{R}^{N}$ centrada em zero e $\alpha>0$. É mostrado que para $p \geq 2_{\alpha}^{*}$, onde

$$
2_{\alpha}^{*}=\left\{\begin{array}{rl}
\frac{2(N+\alpha)}{N-2} ; & N>2, \\
\infty ; & N=1,2,
\end{array} \quad 2^{*}=\left\{\begin{aligned}
\frac{2 N}{N-2} ; & N>2 \\
\infty ; & N=1,2
\end{aligned}\right.\right.
$$

o problema não tem solução não trivial. Em contrapartida, para $1<p<2_{\alpha}^{*}$ com $p \neq 2$, a existência de uma solução positiva radial é garantida. Além disso, é provado a unicidade de solução positiva no caso em que $1<p<2$. Também são apresentados resultados sobre a existência de soluções ground state quando $2<p<2^{*}$. Nesse intervalo, é mostrado que qualquer solução ground state exibe a simetria Schwarz folheada e, no caso em que $\alpha$ é suficientemente grande, é provado que qualquer solução ground state não é radialmente simétrica. Por fim, é apresentado um resultado sobre a existência de múltiplas soluções positivas. 
viii 


\section{Abstract}

This work presents a quantitative and qualitative study of positive solutions for the Dirichlet problem for the Hénon equation

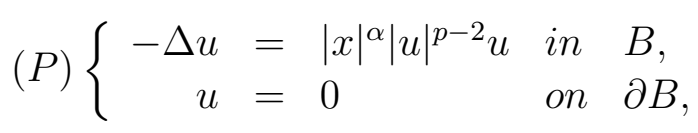

where $B$ is the unit open ball in $\mathbb{R}^{N}$ centered at zero and $\alpha>0$. It is shown that for $p \geq 2_{\alpha}^{*}$, where

$$
2_{\alpha}^{*}=\left\{\begin{array}{rl}
\frac{2(N+\alpha)}{N-2} ; & N>2, \\
\infty ; & N=1,2,
\end{array} \quad 2^{*}=\left\{\begin{aligned}
\frac{2 N}{N-2} ; & N>2, \\
\infty ; & N=1,2,
\end{aligned}\right.\right.
$$

the problem does not have nontrivial solution. In counterpart, for $1<p<2_{\alpha}^{*}$ with $p \neq 2$, the existence of radial positive solutions will be guaranteed. Moreover, the uniqueness of positive solution is guaranteed as long as $1<p<2$. In addition, results on the existence of ground state solutions are presented in case $2<p<2^{*}$. In this interval, it is proved that any ground state solution exhibits the Foliated Schwarz symmetry and, in case $\alpha$ is sufficiently large, it is shown that the no ground state solution is radially symmetric. This works ends with a result on the existence of multiple positive solutions. 


\section{Sumário}

Introdução 2

Notações

1 Identidade de Pohozaev: um resultado de não-existência 5

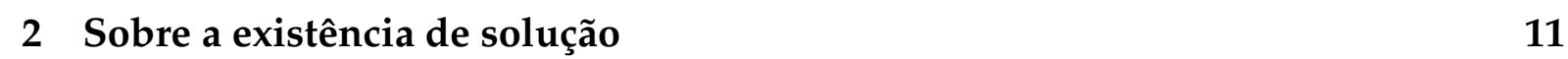

2.1 Lemas radias e de compacidade . . . . . . . . . . . . . . . . 11

2.2 Existência de solução . . . . . . . . . . . . . . . . . . . . 18

2.3 Resultado de regularidade . . . . . . . . . . . . . . . . . 28

2.4 Solução radial para equação de Hénon . . . . . . . . . . . . . . . . . . . 29

2.5 Caracterizações das soluções ground state . . . . . . . . . . . . . . . . . 33

\begin{tabular}{|lll}
\hline 3 & O caso $1<p<2$ & 37
\end{tabular}

\begin{tabular}{|lll}
4 & Quebra de simetria dos ground states & 41
\end{tabular}

4.1 Solução não-radial para equação de Hénon . . . . . . . . . . . . . . . . . 41

4.2 Simetria de Schwarz folheada . . . . . . . . . . . . . . . . 45

5 Sobre a multiplicidade

\begin{tabular}{lll}
\hline A Resultados Básicos & 61
\end{tabular}

A.1 Os Espações de Sobolev $W^{1, p}(I)$ e $W^{k, p}(\Omega) \ldots \ldots \ldots \ldots$. . . . . . . 63

A.2 Resultados sobre existência de solução clássica . . . . . . . . . . . . . . 65

A.3 Princípio geral minimax . . . . . . . . . . . . . . . 66

A.4 O Teorema da Passo da Montanha . . . . . . . . . . . . . . . . 66

A.5 Operador de Nemytskii . . . . . . . . . . . . . . . . . 67 
\begin{tabular}{llr}
\hline B & Resultados técnicos & 69 \\
\hline
\end{tabular} 


\section{Introdução}

\section{A equação}

$$
-\Delta u=|x|^{\alpha}|u|^{p-2} u, \quad \alpha>0
$$

foi introduzida por Hénon [19] como um modelo para o estudo de constelações esfericamente simétricas. Depois do trabalho de Ni [23] de 1982, o problema de Dirichlet para equação de Hénon

$$
-\Delta u=|x|^{\alpha}|u|^{p-2} u \quad \text { in } \quad B, \quad u=0 \quad \text { on } \quad \partial B, \quad \alpha>0, \quad p>1,
$$

onde $B$ é a bola unitária aberta de $\mathbb{R}^{N}$ centrada em zero, $N \geq 1$, tem sido objeto de pesquisa de muitos matemáticos. Em [23], sob a condição $N \geq 3$, Ni mostrou que o clássico expoente crítico de Sobolev $2^{*}$ para este tipo de problema é mudado para um valor mais alto, precisamente $2_{\alpha}^{*}$. Ni observou que o peso $|x|^{\alpha}$ interfere na identidade de Pohožaev, a qual por sua vez garante a não-existência de solução não-trivial para (1) no intervalo $\left[2_{\alpha}^{*}, \infty\right)$. Em contrapartida, através de um Lema Radial, Ni provou que (1) possui uma solução positiva sempre que $2<p<2_{\alpha}^{*}$. Assim, $2_{\alpha}^{*}$ é de fato um limitante para a questão de existência de solução para (1). Estes resultados serão apresentados nos Capítulos 1 e 2 desta dissertação.

Posteriormente, em 2002, Smets, Su e Willem [25] publicaram um artigo muito interessante sobre o problema (1). Uma vez que $|x|^{\alpha}$ é crescente como uma função de $|x|$, a técnica dos planos móveis de [17] não pode ser aplicada para provar que toda solução positiva de (1) é radialmente simétrica. Na verdade, foi provado em [25] para o caso $N \geq 2$, e em [9] para $N \geq 1$, que as soluções de (1) de energia mínima possuem sinal definido, mas não são radialmente simétricas quando $\alpha$ é suficientemente grande. Logo após, nos artigos [26, 24], foi provado que estas soluções de energia mínima, também chamadas de soluções "Ground State"possuem uma simetria residual, a chamada simetria de Schwarz folheada. Este é o assunto do Capítulo 4 deste texto.

A discussão acima motiva-nos a introduzir uma outra questão acerca do problema 
(1), a questão sobre a multiplicidade de soluções positivas. Este é objeto de estudo tratado nos Capítulos 3 e 5 deste material. Primeiramente, no Capítulo 3, seguindo idéias contidas em [22], é mostrado que (1) possui uma única solução positiva quando $1<p<2$, isto é, quando (1) é sublinear. No Capítulo 5, os resultados contidos em [3] são apresentados, e fornecem um resultado de multiplicidade de soluções positivas para (1) no caso superlinear incluindo casos onde $p>2^{*}$. 


\section{Notações}

1. $\frac{\partial u}{\partial x_{i}}$ denota a derivada parcial da função $u$ em relação a $i$-ésima coordenada. Algumas vezes, visando simplificar a notação, $u_{i}$ será usado para representar $\frac{\partial u}{\partial x_{i}}$.

2. $\nabla u=\left(\frac{\partial u}{\partial x_{1}}, \frac{\partial u}{\partial x_{2}}, \ldots, \frac{\partial u}{\partial x_{n}}\right)$ denota o gradiente de $u$.

3. $\Delta u=\sum_{i=1}^{n} \frac{\partial^{2} u}{\partial x_{i}^{2}}$, denota o Laplaciano de $u$.

4. $\frac{\partial u}{\partial \eta}(x)=\partial_{\eta} u(x)=\eta(x) \cdot \nabla u(x)$ denota a derivada normal exterior da função $u$ no ponto $x \in \partial B . \eta(x)$ denota o vetor normal exterior a $\partial B$ no ponto $x \in \partial B$.

5. $\liminf \operatorname{in}_{n \rightarrow \infty} f$ denota o limite inferior da função $f$ quando $n \longrightarrow \infty$.

6. $C_{0}(\bar{\Omega})$ é o conjunto de funções contínuas em $\bar{\Omega}$ que se anulam sobre $\partial \Omega$.

7. $C^{k}(\Omega)$ é o conjunto de funções $k$ vezes continuamente diferenciáveis em $\Omega$.

8. $C^{k}(\bar{\Omega})$ é o conjunto de funções em $C^{k}(\Omega)$, cujas derivadas de ordem $\leq k$ tem extensões continuas sobre $\bar{\Omega}$.

9. $C_{0}^{k}(\bar{\Omega})=C^{k}(\bar{\Omega}) \cap C_{0}(\bar{\Omega})$.

10. $C_{0}^{\infty}(\bar{\Omega})=C^{\infty}(\bar{\Omega}) \cap C_{0}(\bar{\Omega})$.

11. $C^{\infty}(\bar{\Omega})=\bigcap_{k \geq 0} C^{k}(\bar{\Omega})$.

12. $C_{c}^{\infty}(\Omega)$ denota o espaço das funções infinitamente diferenciáveis $\varphi: \Omega \rightarrow \mathbb{R}$ com suporte compacto em $\Omega$. Dotaremos $C_{c}^{\infty}(\Omega)$ com a topologia limite indutivo $\mathcal{L}$. 
13. $C^{0, \alpha}(\bar{\Omega})=\left\{u \in C(\bar{\Omega}) ; \sup _{\substack{x, y \in \Omega \\ x \neq y}} \frac{|u(x)-u(y)|}{|x-y|^{\alpha}}<\infty\right\}$ com $0<\alpha \leq 1$.

14. $C^{k, \alpha}(\bar{\Omega})=\left\{u \in C^{k}(\Omega) ; D^{j} u \in C^{0, \alpha}(\bar{\Omega}), \forall j,|j| \leq k\right\}$.

15. $\langle$,$\rangle denota a aplicação dualidade.$ 


\section{Capítulo}

1

\section{Identidade de Pohozaev: um resultado de não-existência}

Neste capítulo será provado um resultado geral que será aplicado para provar a não-existência de solução não trivial para o problema de Dirichlet relativo à equação de Hénon,

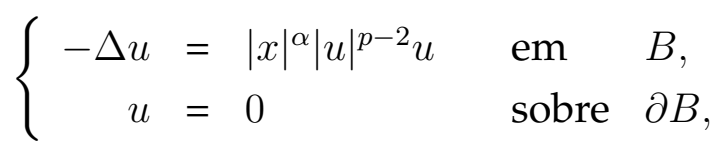

onde $B$ denota a bola unitária aberta de $\mathbb{R}^{N}, \alpha>0, N>2$ e $p \geq \frac{2(N+\alpha)}{N-2}$.

Teorema 1.1. [13, Lemma 1.1] Sejam $\Omega \subset \mathbb{R}^{N}$ um domínio limitado com fronteira suave $e$ $g: \bar{\Omega} \times \mathbb{R} \rightarrow \mathbb{R}$ uma função contínua. Se $u \in C^{2}(\bar{\Omega})$ satisfaz

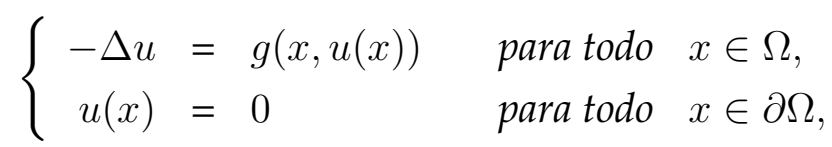

$y \in \mathbb{R}^{N}$ é um vetor fixado e $\eta(x)$ denota o vetor normal unitário exterior a $\partial \Omega$ no ponto $x$, então u satisfaz a seguinte identidade:

$$
\begin{aligned}
\int_{\partial \Omega}|\nabla u(x)|^{2}[(x-y) \cdot \eta(x)] d x & =2 N \int_{\Omega} G(x, u) d x-(N-2) \int_{\Omega} g(x, u) u d x \\
& +2 \sum_{i=1}^{N} \int_{\Omega}\left(x_{i}-y_{i}\right) G_{x_{i}}(x, u) d x
\end{aligned}
$$

onde $G(x, s)=\int_{0}^{s} g(x, t) d t$. 
Demonstração. Sendo $u$ uma solução do problema (1.2) segue-se que

$$
\begin{aligned}
-\int_{\Omega} \Delta u[(x-y) \cdot \nabla u] d x & =\int_{\Omega} g(x, u(x))[(x-y) \cdot \nabla u] d x \\
& =\int_{\Omega}\left[g(x, u(x)) \sum_{i=1}^{N}\left(x_{i}-y_{i}\right) \frac{\partial u}{\partial x_{i}}\right] d x \\
& =\sum_{i=1}^{N} \int_{\Omega}\left[\left(x_{i}-y_{i}\right) g(x, u(x)) \frac{\partial u}{\partial x_{i}}\right] d x
\end{aligned}
$$

Usando o Teorema de Integração por partes (ver Corolário A.4), obtém-se

$$
\int_{\Omega}\left(x_{i}-y_{i}\right) \frac{\partial G}{\partial x_{i}}(x, u(x)) d x=-\int_{\Omega} G(x, u(x)) d x .
$$

Pela regra da cadeira note que

$$
\frac{\partial}{\partial x_{i}} G(x, u(x))=G_{x_{i}}(x, u(x))+G_{u}(x, u(x)) \frac{\partial u}{\partial x_{i}}=G_{x_{i}}(x, u(x))+g(x, u(x)) \frac{\partial u}{\partial x_{i}} .
$$

Assim, as igualdades (1.4), (1.6) e (1.5) implicam em

$$
\begin{aligned}
-\int_{\Omega} \Delta u[(x-y) \cdot \nabla u] d x & =\sum_{i=1}^{N} \int_{\Omega}\left(x_{i}-y_{i}\right)\left[\frac{\partial G}{\partial x_{i}}(x, u(x))-G_{x_{i}}(x, u(x))\right] d x \\
& =\sum_{i=1}^{N}\left[\int_{\Omega}\left(x_{i}-y_{i}\right) \frac{\partial G}{\partial x_{i}}(x, u(x))-\int_{\Omega}\left(x_{i}-y_{i}\right) G_{x_{i}}(x, u(x))\right] d x \\
& =-N \int_{\Omega} G(x, u(x)) d x-\sum_{i=1}^{N} \int_{\Omega}\left(x_{i}-y_{i}\right) G_{x_{i}}(x, u(x)) d x .
\end{aligned}
$$

Agora calcula-se o divergente do campo $[(x-y) \cdot \nabla u] \nabla u$. Isto é

$$
\begin{aligned}
\operatorname{div}\{[(x-y) \cdot \nabla u] \nabla u\}= & \operatorname{div}\left([(x-y) \cdot \nabla u] \frac{\partial u}{\partial x_{1}}, \ldots,[(x-y) \cdot \nabla u] \frac{\partial u}{\partial x_{N}}\right) \\
= & \frac{\partial}{\partial x_{1}}\left([(x-y) \cdot \nabla u] \frac{\partial u}{\partial x_{1}}\right)+\ldots+\frac{\partial}{\partial x_{N}}\left([(x-y) \cdot \nabla u] \frac{\partial u}{\partial x_{N}}\right) \\
= & {\left[\frac{\partial}{\partial x_{1}}[(x-y) \cdot \nabla u] \frac{\partial u}{\partial x_{1}}+[(x-y) \cdot \nabla u] \frac{\partial^{2} u}{\partial x_{1}^{2}}\right]+\ldots } \\
& \ldots+\left[\frac{\partial}{\partial x_{N}}[(x-y) \cdot \nabla u] \frac{\partial u}{\partial x_{N}}+[(x-y) \cdot \nabla u] \frac{\partial^{2} u}{\partial x_{N}^{2}}\right] \\
= & {\left[\frac{\partial}{\partial x_{1}}[(x-y) \cdot \nabla u] \frac{\partial u}{\partial x_{1}}+\ldots+\frac{\partial u}{\partial x_{N}}[(x-y) \cdot \nabla u] \frac{\partial^{2} u}{\partial x_{N}}\right]+} \\
& +[(x-y) \cdot \nabla u]\left(\frac{\partial^{2} u}{\partial x_{1}^{2}}+\ldots+\frac{\partial^{2}}{\partial x_{N}^{2}}\right)
\end{aligned}
$$




$$
\begin{aligned}
= & \frac{\partial}{\partial x_{1}}\left(\sum_{i=1}^{N}\left(x_{i}-y_{i}\right) \frac{\partial u}{\partial x_{i}}\right) \frac{\partial u}{\partial x_{1}}+\ldots+\frac{\partial}{\partial x_{N}}\left(\sum_{i=1}^{N}\left(x_{i}-y_{i}\right) \frac{\partial u}{\partial x_{i}}\right) \frac{\partial u}{\partial x_{N}} \\
& +[(x-y) \cdot \nabla u] \Delta u \\
= & {\left[\sum_{k=1}^{N} \frac{\partial}{\partial x_{k}}\left(\sum_{i=1}^{N}\left(x_{i}-y_{i}\right) \frac{\partial u}{\partial x_{i}}\right) \frac{\partial u}{\partial x_{k}}\right]+[(x-y) \cdot \nabla u] \Delta u } \\
= & \sum_{k=1}^{N}\left(\frac{\partial u}{\partial x_{k}}+\sum_{i=1}^{N}\left(x_{i}-y_{i}\right) \frac{\partial^{2} u}{\partial x_{k} \partial x_{i}}\right) \frac{\partial u}{\partial x_{k}}+[(x-y) \cdot \nabla u] \Delta u \\
= & {[(x-y) \cdot \nabla u] \Delta u+\sum_{k=1}^{N}\left(\frac{\partial u}{\partial x_{k}}\right)^{2}+\sum_{k=1}^{N}\left[\sum_{i=1}^{N}\left(x_{i}-y_{i}\right) \frac{\partial^{2} u}{\partial x_{k} \partial x_{i}}\right] \frac{\partial u}{\partial x_{k}} } \\
= & {[(x-y) \cdot \nabla u] \Delta u+|\nabla u|^{2}+\sum_{i, k=1}^{N}\left(x_{i}-y_{i}\right) \frac{\partial u}{\partial x_{k}} \frac{\partial^{2} u}{\partial x_{k} \partial x_{i}} } \\
= & {[(x-y) \cdot \nabla u] \Delta u+|\nabla u|^{2}+\sum_{i, k=1}^{N}\left(x_{i}-y_{i}\right) \frac{1}{2} \frac{\partial}{\partial x_{i}}\left[\left(\frac{\partial u}{\partial x_{k}}\right)^{2}\right] } \\
= & {[(x-y) \cdot \nabla u] \Delta u+|\nabla u|^{2}+\frac{1}{2} \sum_{i=1}^{N}\left(x_{i}-y_{i}\right) \frac{\partial}{\partial x_{i}}|\nabla u|^{2} . }
\end{aligned}
$$

Usando o Teorema do Divergente, isto é Teorema A.3, juntamente com a igualdade (1.8) conclui-se que:

$$
\begin{aligned}
\int_{\partial \Omega}[(x-y) \cdot \nabla u] \nabla u \cdot \eta(x) d x= & \int_{\Omega}[(x-y) \cdot \nabla u] \Delta u d x+\int_{\Omega}|\nabla u|^{2} d x+ \\
& +\frac{1}{2} \int_{\Omega} \sum_{i=1}^{N}\left(x_{i}-y_{i}\right) \frac{\partial}{\partial x_{i}}|\nabla u|^{2} d x .
\end{aligned}
$$

Por outro lado, o divergente do campo $\frac{1}{2}|\nabla u|^{2}(x-y)$ é

$$
\begin{aligned}
\operatorname{div}\left[\frac{1}{2}|\nabla u|^{2}(x-y)\right] & =\operatorname{div}\left(\frac{x_{1}-y_{1}}{2}|\nabla u|^{2}, \ldots, \frac{x_{N}-y_{N}}{2}|\nabla u|^{2}\right) \\
& =\frac{\partial}{\partial x_{1}}\left(\frac{x_{1}-y_{1}}{2}|\nabla u|^{2}\right)+\ldots+\frac{\partial}{\partial x_{N}}\left(\frac{x_{N}-y_{N}}{2}|\nabla u|^{2}\right) \\
= & \left(\frac{1}{2}|\nabla u|^{2}+\frac{x_{1}-y_{1}}{2} \frac{\partial}{\partial x_{1}}|\nabla u|^{2}\right)+\ldots+\left(\frac{1}{2}|\nabla u|^{2}+\frac{x_{N}-y_{N}}{2} \frac{\partial}{\partial x_{N}}|\nabla u|^{2}\right) \\
= & \frac{N}{2}|\nabla u|^{2}+\frac{1}{2} \sum_{i=1}^{N}\left(x_{i}-y_{i}\right) \frac{\partial}{\partial x_{i}}|\nabla u|^{2} .
\end{aligned}
$$


Usando o Teorema Divergente juntamente com a identidade (1.10), acarreta

$$
\frac{1}{2} \int_{\partial \Omega}|\nabla u|^{2}[(x-y) \cdot \eta(x)] d x=\frac{N}{2} \int_{\Omega}|\nabla u|^{2} d x+\frac{1}{2} \int_{\Omega} \sum_{i=1}^{N}\left(x_{i}-y_{i}\right) \frac{\partial}{\partial x_{i}}|\nabla u|^{2} d x .
$$

Das igualdades dadas em (1.9) e (1.11) segue-se

$$
\begin{aligned}
\int_{\partial \Omega}[(x-y) \cdot \nabla u] \nabla u \cdot \eta(x) d x & =\int_{\Omega} \Delta u[(x-y) \cdot \nabla u] d x+\int_{\Omega}|\nabla u|^{2} d x \\
& +\frac{1}{2} \int_{\partial \Omega}|\nabla u|^{2}[(x-y) \cdot \eta(x)] d x-\frac{N}{2} \int_{\Omega}|\nabla u|^{2} d x .
\end{aligned}
$$

Assim

$$
\begin{aligned}
\int_{\partial \Omega}[(x-y) \cdot \nabla u] \nabla u \cdot \eta(x) d x & =\frac{1}{2} \int_{\partial \Omega}|\nabla u|^{2}(x-y) \cdot \eta(x) d x+\frac{2-N}{2} \int_{\Omega}|\nabla u|^{2} d x \\
& +\int_{\Omega} \Delta u[(x-y) \cdot \nabla u] d x .
\end{aligned}
$$

Também visto que $\nabla u(x)$ é paralelo a $\eta(x)$ para todo $x \in \partial \Omega$, existe $\lambda(x) \in \mathbb{R}$ tal que

$$
\nabla u(x)=\lambda(x) \cdot \eta(x) \Longrightarrow \nabla u(x) \cdot \eta(x)=\lambda(x) \eta(x) \cdot \eta(x)=\lambda(x)|\eta(x)|^{2}=\lambda(x)
$$

Logo

$$
\begin{aligned}
\nabla u(x) & =(\nabla u \cdot \eta(x)) \eta(x) \\
|\nabla u(x)|^{2} & =|(\nabla u \cdot \eta(x)) \eta(x)|^{2}=|\nabla u \cdot \eta(x)|^{2} .
\end{aligned}
$$

Segue-se destas duas últimas igualdades que

$$
\begin{aligned}
{[(x-y) \cdot \nabla u](\nabla u \cdot \eta(x)) } & =[(x-y) \cdot((\nabla u \cdot \eta(x)) \eta(x))](\nabla u \cdot \eta(x)) \\
& =[(x-y) \cdot \eta(x)]|\nabla u \cdot \eta(x)|^{2} \\
& =[(x-y) \cdot \eta(x)]|\nabla u|{ }^{2}
\end{aligned}
$$

Integrando sobre a fronteira $\partial B$ e tendo em vista (1.12) resulta

$$
\begin{aligned}
\int_{\partial \Omega}[(x-y) \cdot \eta(x)]|\nabla u|^{2} d x & =\frac{1}{2} \int_{\partial \Omega}|\nabla u|^{2}[(x-y) \cdot \eta(x)] d x+\frac{2-N}{2} \int_{\Omega}|\nabla u|^{2} d x \\
& +\int_{\Omega} \Delta u[(x-y) \cdot \nabla u] d x
\end{aligned}
$$

ou equivalentemente,

$$
\int_{\partial \Omega}|\nabla u|^{2}[(x-y) \cdot \eta(x)] d x=2 \int_{\Omega} \Delta u[(x-y) \cdot \nabla u] d x+(2-N) \int_{\Omega}|\nabla u|^{2} d x .
$$


De (1.7) e 1.13) conclui-se

$$
\begin{aligned}
\int_{\partial \Omega}|\nabla u|^{2}[(x-y) \cdot \eta(x)] d x= & 2 N \int_{\Omega} G(x, u(x)) d x+2 \sum_{i=1}^{N} \int_{\Omega}\left(x_{i}-y_{i}\right) G_{x_{i}}(x, u(x)) d x \\
& -(N-2) \int_{\Omega}|\nabla u|^{2} d x
\end{aligned}
$$

e desde que $u$ é solução do problema (1.2), tem-se

$$
\int_{\Omega}|\nabla u|^{2} d x=\int_{\Omega} g(x, u) u d x .
$$

Logo de isto segue que

$$
\begin{aligned}
\int_{\partial \Omega}|\nabla u|^{2}[(x-y) \cdot \eta(x)] d x= & 2 N \int_{\Omega} G(x, u(x)) d x-(N-2) \int_{\Omega} g(x, u) u d x . \\
& +2 \sum_{i=1}^{N} \int_{\Omega}\left(x_{i}-y_{i}\right) G_{x_{i}}(x, u(x)) d x
\end{aligned}
$$

Portanto o teorema está demonstrado.

Teorema 1.2. Seja $N \geq 3$. Então o problema (1.1) não tem solução não trivial $u \in C^{2}(\bar{B})$ para $p \geq 2_{\alpha}^{*}$.

Demonstração. Seja $u \in C^{2}(\bar{B})$ solução não trivial de $(1.1)$. Considerando

$$
g(x, s)=|x|^{\alpha}|s|^{p-2} s, G(x, s)=\int_{0}^{s} g(x, t) d t=\frac{|x|^{\alpha}|s|^{p}}{p}
$$

e observando que $G_{x_{i}}(x, s)=\frac{\alpha|x|^{\alpha-2} x_{i}|s|^{p}}{p}$ no Teorema 1.1 (para $\left.y=0\right)$ tem-se,

$$
\begin{aligned}
\int_{\partial B}|\nabla u|^{2}[x \cdot \eta(x)] d x= & 2 N \int_{B} \frac{|x|^{\alpha}|u|^{p}}{p} d x-(N-2) \int_{B}|x|^{\alpha}|u|^{p} d x \\
& +2 \sum_{i=1}^{N} \int_{B} \frac{x_{i}^{2} \alpha|x|^{\alpha-2}|u|^{p}}{p} d x
\end{aligned}
$$

Por outro lado, para cada $x \in \partial B, \eta(x)=x$, isto implica

$$
\int_{\partial B}|\nabla u(x)|^{2}[x \cdot \eta(x)] d x=\int_{\partial B}|\nabla u(x)|^{2} d x \geq 0 .
$$

Se

$$
\int_{\partial B}|\nabla u(x)|^{2} d x=0,
$$


desde que $u \in C^{2}(\bar{B})$ tem-se $\nabla u \equiv 0$. Além disso $u \equiv 0$ sobre $\partial B$. Logo pelo Princípio da Continuação Única (veja [27, pg. 172]) tem-se $u \equiv 0$ em $B$, o que é uma contradição. Portanto

$$
\int_{\partial B}|\nabla u(x)|^{2}[x \cdot \eta(x)] d x>0 .
$$

Note que sendo $u$ solução do problema (1.1), este satisfaz

$$
\int_{B} \nabla u \cdot \nabla v d x=\int_{B}|x|^{\alpha}|u|^{p-2} u v d x, \quad \forall v \in H_{0}^{1}(B) .
$$

E fazendo $v=u$,

$$
\int_{B}|\nabla u|^{2} d x=\int_{B}|x|^{\alpha}|u|^{p} d x>0
$$

Segue-se de isto juntamente com as expressões (1.14)-(1.15) que

$$
\begin{aligned}
& 0<\frac{2 N}{p} \int_{B}|x|^{\alpha}|u|^{p} d x-(N-2) \int_{B}|x|^{\alpha}|u|^{p} d x+2 \sum_{i=1}^{N} \int_{B} \frac{x_{i}^{2} \alpha|x|^{\alpha-2}|u|^{p}}{p} d x \\
&=\frac{2 N}{p} \int_{B}|x|^{\alpha}|u|^{p} d x-(N-2) \int_{B}|x|^{\alpha}|u|^{p} d x+\frac{2 \alpha}{p} \sum_{i=1}^{N} \int_{B} x_{i}^{2}|x|^{\alpha-2}|u|^{p} d x \\
& \leq\left(\frac{2 N}{p}-(N-2)\right) \int_{B}|x|^{\alpha}|u|^{p} d x+\frac{2 \alpha}{p} \int_{B}|x|^{2}|x|^{\alpha-2}|u|^{p} d x \\
&=\left(\frac{2(N+\alpha)}{p}-(N-2)\right) \int_{B}|x|^{\alpha}|u|^{p} d x . \\
& \text { Como } \int_{B}|x|^{\alpha}|u|^{p} d x>0, \text { então }\left(\frac{2(N+\alpha)}{p}-(N-2)\right)>0 . \text { Logo segue-se que } \\
& \quad p<\frac{2(N+\alpha)}{N-2}=2_{\alpha}^{*} .
\end{aligned}
$$

Assim, o teorema está demostrado. 


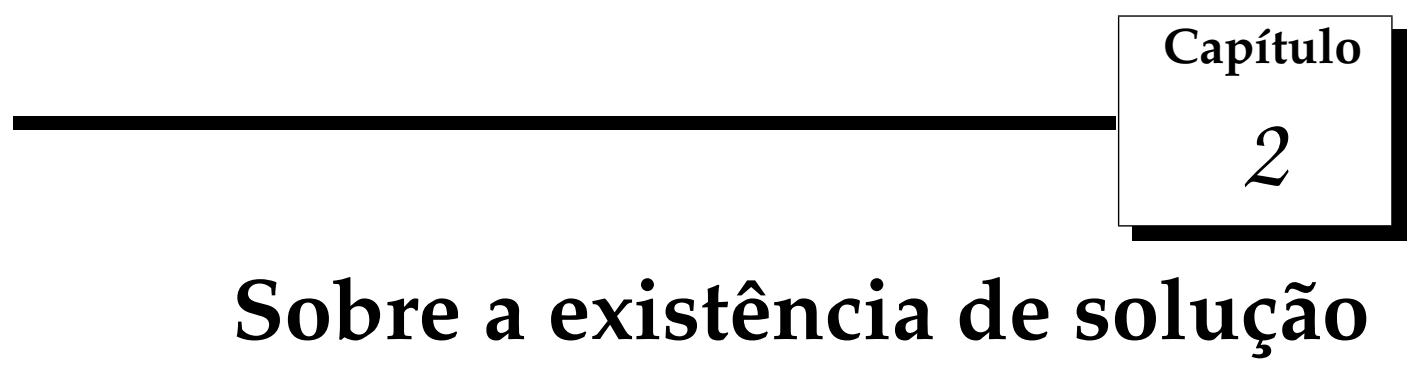

O objetivo deste capítulo é provar a existência de solução positiva para o problema de Dirichlet para a equação de Hénon

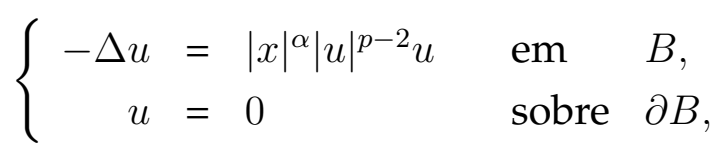

no caso quando $1<p<2_{\alpha}^{*}$ e $p \neq 2$. Este resultado é ótimo, uma vez que no capítulo anterior foi provada a não-existência de solução não-trivial no caso em que $p \geq 22_{\alpha}^{*}$. Aqui também é mostrado a existência de solução de menor energia entre todas as soluções não-triviais no caso em que $1<p<2^{*}$, com $p \neq 2$, e a existência de solução radial de menor energia entre todas as soluções radiais não-triviais no caso em que $1<p<2_{\alpha}^{*}$, com $p \neq 2$. Também será mostrado que estas soluções de energia mímina tem sinal definido, isto é, ou são positivas ou são negativas em todo $B$.

Observe que no caso $p=2$ a equação (2.1) é uma equação linear. Assim $\operatorname{com} p=2$ a equação de Hénon tem solução positiva se, e somente se, 1 é o primeiro autovalor do problema

$$
-\Delta u=\lambda|x|^{\alpha} u \text { em } B, \quad u=0 \text { em } \partial B
$$

Por isso no que segue supõe-se $1<p<2_{\alpha}^{*}$ e $p \neq 2$.

\subsection{Lemas radias e de compacidade}

Nesta seção são provados alguns lemas que são necessários para mostrar a existência de solução radial para a equação (2.1). 
Uma função radial definida em $B$ pode ser vista como uma função definida em $(0,1)$. Associada a $u \in W_{\text {rad }}^{1, p}(B)$ tem-se uma função $v$ definida em $(0,1)$ da seguinte forma: $v(t):=u(x) \operatorname{com} t=|x|$.

Dado um número real $p \geq 1$ seja $W^{1, p}\left((0,1), t^{N-1}\right)$ o espaço de Sobolev com peso,

$$
\left\{v:(0,1) \rightarrow \mathbb{R} ; v \text { tem derivada fraca e } \int_{0}^{1}\left|v^{(j)}(t)\right|^{p} t^{N-1} d t<\infty ; j=0,1\right\}
$$

dotado com a norma

$$
\|v\|_{W_{N-1}^{1, p}}=\left(\int_{0}^{1}\left(|v(t)|^{p}+\left|v^{\prime}(t)\right|^{p}\right) t^{N-1} d t\right)^{1 / p} ; \quad v \in W^{1, p}\left((0,1), t^{N-1}\right) .
$$

Teorema 2.1. Seja $u \in W^{1, p}(I) \operatorname{com} 1 \leq p \leq \infty$, I limitado ou ilimitado. Então existe uma função $\tilde{u} \in C(\bar{I})$ tal que $u=\tilde{u}$ q.t.p. em $I$ e

$$
\tilde{u}(x)-\tilde{u}(y)=\int_{y}^{x} u^{\prime}(t) d t, \quad \forall x, y \in \bar{I} .
$$

Demonstração. Veja [8, Theorem 8.2].

Proposição 2.2. Do teorema acima, $v \in W^{1, p}\left((0,1), t^{N-1}\right)$ se, somente se, existe $V \in C((0,1])$ tal que $V^{\prime}(t)$ (no sentido clássico) existe em q.t.p. $t \in(0,1), V^{\prime}(t)$ é função mensurável, $v=V$, $v^{\prime}=V^{\prime}$ q.t.p. em $(0,1) e \int_{0}^{1}\left|v^{(j)}(t)\right|^{p} t^{N-1} d t<\infty$, para $j=0,1$.

Demonstração. Seja $v \in W^{1, p}\left((0,1), t^{N-1}\right)$, então $v \in W^{1, p}(\epsilon, 1)$ para qualquer $0<\epsilon<1$ e $\int_{0}^{1}\left|v^{(j)}(t)\right|^{p} t^{N-1} d t<\infty, j=0,1$. Pelo teorema anterior, existe $V \in C((0,1])$ tal que $v=V$ q.t.p. em $(0,1)$ e $v^{\prime}=V^{\prime}$ q.t.p. em $(0,1)$.

Reciprocamente, se existe $V \in C((0,1])$ com as hipóteses mencionadas acima, então $V \in W^{1, p}\left((0,1), t^{N-1}\right)$.

Em vista a Proposição 2.2. qualquer elemento $v \in W^{1, p}\left((0,1), t^{N-1}\right)$ será substituído pelo seu representante $V$ como acima, mas a notação $v$ será mantida. Os teoremas a seguir mostram uma precisa relação entre $W_{\text {rad }}^{1, p}(B)$ e $W^{1, p}\left((0,1), t^{N-1}\right)$.

Teorema 2.3. [12. Theorem 2.2] Se $u \in W_{\text {rad }}^{1, p}(B)$, então $v \in W^{1, p}\left((0,1), t^{N-1}\right)$, onde $v$ é definido por $v(t):=u(x) \operatorname{com} t=|x|$.

Demonstração. Seja uma função $u \in W_{\text {rad }}^{1, p}(B)$. Dada $\varphi \in C_{c}^{1}(0,1)$ considere $\psi(x):=$ 
$\varphi(t) \operatorname{com} t=|x|, x \in B$. Note que

$$
\psi_{x_{i}}(x)=\frac{\partial}{\partial x_{i}}(\varphi(|x|))=\varphi^{\prime}(|x|) \frac{x_{i}}{|x|}, \quad \forall x \in B \backslash\{0\}
$$

Disto, segue-se que

$$
\sum_{i=1}^{N} \psi_{x_{i}}(x) \frac{x_{i}}{|x|}=\varphi^{\prime}(|x|)
$$

Observe que

$$
\operatorname{div}\left(\frac{x}{|x|^{N}}\right)=\sum_{i=1}^{N} \frac{\partial}{\partial x_{i}}\left(\frac{x_{i}}{|x|^{N}}\right)=0 .
$$

Agora, usando (2.2) tem-se

$$
\begin{aligned}
\int_{0}^{1} v(t) \varphi^{\prime}(t) d t & =\frac{1}{\omega_{N}} \int_{0}^{1} \frac{v(r) \varphi^{\prime}(r)}{r^{N-1}} \omega_{N} r^{N-1} d r=\frac{1}{\omega_{N}} \int_{0}^{1} \frac{v(r) \varphi^{\prime}(r)}{r^{N-1}}\left|S_{r}\right| d r \\
& =\frac{1}{\omega_{N}} \int_{0}^{1} \int_{S_{r}} \frac{u(x) \varphi^{\prime}(|x|)}{|x|^{N-1}} d S(x) d r=\frac{1}{\omega_{N}} \int_{B} \frac{u(x) \varphi^{\prime}(|x|)}{|x|^{N-1}} d x \\
& =\frac{1}{\omega_{N}} \int_{B}\left(\sum_{i=1}^{N} u(x) \frac{x_{i}}{|x|^{N}} \psi_{x_{i}}(x)\right) d x,
\end{aligned}
$$

onde $\omega_{N}$ é a área de superfície da esfera unitária $S^{N-1} \subset \mathbb{R}^{N}$. Por outro lado, para cada $i \in\{1,2, \ldots, N\}$,

$$
\begin{aligned}
\int_{B}\left(\frac{x_{i}}{|x|^{N}} u(x)\right) \psi_{x_{i}}(x) d x & =-\int_{B}\left(\frac{\partial}{\partial x_{i}}\left(\frac{x_{i}}{|x|^{N}}\right) u(x)+\frac{x_{i}}{|x|^{N}} u_{x_{i}}(x)\right) \psi(x) d x \\
& =-\int_{B} \psi(x) \frac{\partial}{\partial x_{i}}\left(\frac{x_{i}}{|x|^{N}}\right) u(x) d x-\int_{B} \psi(x) \frac{x_{i}}{|x|^{N}} u_{x_{i}}(x) d x
\end{aligned}
$$

Assim, usando a expressão (2.3) resulta

$$
\begin{aligned}
\frac{1}{\omega_{N}} \int_{B}\left(\sum_{i=1}^{N} \frac{x_{i}}{|x|^{N}} u(x) \psi_{x_{i}}(x)\right) d x & =-\frac{1}{\omega_{N}} \int_{B} \psi(x) \operatorname{div}\left(\frac{x_{i}}{|x|^{N}}\right) u(x) d x \\
& -\frac{1}{\omega_{N}} \int_{B} \sum_{i=1}^{N} \psi(x) \frac{x_{i}}{|x|^{N}} u_{x_{i}}(x) d x \\
& =-\frac{1}{\omega_{N}} \int_{B}\left(\sum_{i=1}^{N} u_{x_{i}}(x) \frac{x_{i}}{|x|^{N}}\right) \psi(x) d x
\end{aligned}
$$

e portanto, 


$$
\begin{aligned}
\int_{0}^{1} v(t) \varphi^{\prime}(t) d t & =-\frac{1}{w_{N}} \int_{B}\left(\sum_{i=1}^{N} u_{x_{i}}(x) \frac{x_{i}}{|x|^{N}}\right) \psi(x) d x \\
& =-\frac{1}{w_{N}} \int_{B}\left(\sum_{i=1}^{N} u_{x_{i}}(x) \frac{x_{i}}{|x|}\right) \psi(x) \frac{1}{|x|^{N-1}} d x=-\int_{0}^{1} g(t) \varphi(t) d t
\end{aligned}
$$

onde $g(|x|):=\sum_{i=1}^{N} u_{x_{i}}(x) \frac{x_{i}}{|x|}$.

Assim $v$ possui derivada fraca $v^{\prime}$ e $v^{\prime}=g$. Como

$$
\int_{0}^{1}|v(t)|^{p} t^{N-1} d t=\frac{1}{\omega_{N}} \int_{B}|u(x)|^{p} d x \quad \text { e } \quad \int_{0}^{1}\left|v^{\prime}(t)\right|^{p} t^{N-1} d t=\frac{1}{\omega_{N}} \int_{B}|\nabla u(x)|^{p} d x
$$

segue-se que $v \in W^{1, p}\left((0,1), t^{N-1}\right)$. Observe que foi usado o fato de $\left|v^{\prime}(|x|)\right|=|\nabla u(x)|$.

Observação 2.4. Para todo $p \geq 1, W_{\text {rad }}^{1, p}(B) \hookrightarrow W^{1, p}\left((0,1), t^{N-1}\right)$ é uma imersão contínua.

De fato, dada $u \in W_{\text {rad }}^{1, p}(B)$ definimos $v(t)=u(x) \operatorname{com} t=|x|$. Então

$$
\|v\|_{W_{N-1}^{1, p}}^{p}=\int_{0}^{1}\left(|v(t)|^{p}+\left|v^{\prime}(t)\right|^{p}\right) t^{N-1} d t=\frac{1}{\omega_{N}} \int_{B}|u(x)|^{p}+|\nabla u(x)|^{p} d x=\frac{1}{\omega_{N}}\|u\|_{1, p}^{p} .
$$

Lema 2.5. [12, Lemma 2.5] Suponha $N>p \geq 1$. Existe uma constante $C>0$ tal que para todo $v \in W^{1, p}\left((0,1), t^{N-1}\right)$

$$
|v(t)| \leq C \frac{\|v\|_{W_{N-1}^{1, p}}}{t^{\frac{N-p}{p}}}, \quad \forall t \in(0,1]
$$

Demonstração. Considere $p>1$, pois o caso $p=1$ é mais simples uma vez que nem mesmo é necessário aplicar a desigualdade de Hölder e sim apenas a limitação uniforme $s^{1-N} \leq t^{1-N}$ para todo $s \in[t, 1]$ e todo $t \in(0,1]$.

Seja $t \in(0,1]$ e $v \in W^{1, p}\left((0,1), t^{N-1}\right)$. Então

$$
v(t)=v(1)-\int_{t}^{1} v^{\prime}(s) d s=v(1)-\int_{t}^{1} v^{\prime}(s) s^{\frac{N-1}{p}} s^{\frac{1-N}{p}} d s .
$$

Seja

$$
\begin{aligned}
h:\left[\frac{1}{2}, 1\right] & \longrightarrow \mathbb{R} \\
t & \longmapsto h(t)=\int_{a}^{t} v(s) d s, \quad 0<a<\frac{1}{2} .
\end{aligned}
$$


Pelo teorema do valor médio, existe $s_{0} \in\left(\frac{1}{2}, 1\right)$ tal que $h(1)-h\left(\frac{1}{2}\right)=\frac{1}{2} h^{\prime}\left(s_{0}\right)$, isto é,

$$
v\left(s_{0}\right)=2 \int_{1 / 2}^{1} v(s) s^{\frac{N-1}{p}} s^{\frac{1-N}{p}} d s .
$$

Também,

$$
v(1)-v\left(s_{0}\right)=\int_{s_{0}}^{1} v^{\prime}(s) d s=\int_{s_{0}}^{1} v^{\prime}(s) s^{\frac{N-1}{p}} s^{\frac{1-N}{p}} d s .
$$

Agora somando as expressões (2.5)-(2.6) tem-se

$$
|v(1)| \leq 2 \int_{1 / 2}^{1}|v(s)| s^{\frac{N-1}{p}} s^{\frac{1-N}{p}} d s+\int_{s_{0}}^{1}\left|v^{\prime}(s)\right| s^{\frac{N-1}{p}} s^{\frac{1-N}{p}} d s .
$$

Então, por Hölder para os expoentes conjugados $p$ e $\frac{p}{p-1}$, existe uma constante $C$, que depende somente de $1 / 2, \mathrm{~N}$ e $p$ tal que

$$
|v(1)| \leq C\|v\|_{W_{N-1}^{1, p}}
$$

Das expressão (2.4)-(2.7) e usando mais uma vez a desigualdade de Hölder com expoentes conjugados $p$ e $\frac{p}{p-1}$, resulta

$$
\begin{aligned}
|v(t)| & \leq|v(1)|+\left|\int_{t}^{1} v^{\prime}(s) s^{\frac{N-1}{p}} s^{\frac{1-N}{p}} d s\right| \\
& \leq C\|v\|_{W_{N-1}^{1, p}}+\left(\int_{t}^{1}\left|v^{\prime}(s)\right|^{p} s^{N-1} d s\right)^{1 / p}\left(\int_{t}^{1} s^{\frac{1-N}{p-1}} d s\right)^{(p-1) / p} \\
& \leq C\|v\|_{W_{N-1}^{1, p}}+\left(\int_{0}^{1}\left|v^{\prime}(s)\right|^{p} s^{N-1} d s\right)^{1 / p}\left(\int_{t}^{1} s^{\frac{1-N}{p-1}} d s\right)^{(p-1) / p} \\
& \leq C\|v\|_{W_{N-1}^{1, p}}+\|v\|_{W_{N-1}^{1, p}}\left(\int_{t}^{1} s^{\frac{1-N}{p-1}} d s\right)^{(p-1) / p} \leq C \frac{\|v\|_{W_{N-1}^{1, p}}}{t^{\frac{N-p}{p}}}
\end{aligned}
$$

onde $C$ depende somente de $1 / 2, N$ e $p$.

Teorema 2.6 (Espaço de Sobolev radial). [12, Theorem 1.1] Para todo $p \geq 1$ :

(1) Toda função $u \in W_{\mathrm{rad}}^{1, p}(B)$ é igual em quase todo ponto a uma função $U \in C^{0}(\bar{B} \backslash\{0\})$. Além disso $U_{x_{i}}(x)$ existe em q.t.p. $|x| \in(0,1)$.

(2) Se $N>p$, então existe uma constante positiva $C$ tal que para todo $u \in W_{\text {rad }}^{1, p}(B)$

$$
|U(x)| \leq C \frac{\|u\|_{1, p}}{|x|^{\frac{N-p}{p}}}, \quad \forall x \in \bar{B} \backslash\{0\}
$$


Com U como no item (1).

\section{Demonstração.}

(1) Seja $u \in W_{\text {rad }}^{1, p}(B)$, define-se $v(t):=u(x) \operatorname{com} t=|x|$, segue-se do Teorema 2.3 que $v \in W^{1, p}\left((0,1), t^{n-1}\right)$ também pela Proposição 2.2 existem $V \in C^{0}((0,1])$ e $v=V$ q.t.p. em $(0,1)$.

Seja $U(x):=V(|x|)$. Assim definido $U \in C^{0}(\bar{B} \backslash\{0\})$ e $U=u$ q.t.p. em $B$. Além disso, $U_{x_{i}}(x)=V^{\prime}(|x|) \frac{x_{i}}{|x|}=v^{\prime}(|x|) \frac{x_{i}}{|x|}=u_{x_{i}}(x)$.

(2) Seja $u \in W_{\text {rad }}^{1, p}(B)$ procedendo como no item (1) tem-se $U=u$ q.t.p. em $B$. Além disso, $U(x)=v(|x|)$ q.t.p. em $B$ e $v \in W^{1, p}\left((0,1), t^{N-1}\right)$ onde $v(t):=u(x)$ com $t=|x|$. Do Lema 2.5 e da Observação 2.4, obtém-se

$$
|v(t)| \leq C \frac{\|v\|_{W_{N-1}^{1,2}}}{t^{\frac{N-p}{p}}} \leq C \frac{\|u\|_{1, p}}{t^{\frac{N-p}{p}}} .
$$

Assim,

$$
|U(x)| \leq C \frac{\|u\|_{1, p}}{|x|^{\frac{N-p}{p}}} .
$$

Observação: Note que cada função $u \in W_{\text {rad }}^{1, p}(B)$ admite um e apenas um representante contínuo em $\bar{B} \backslash\{0\}$, i.e., existe uma função contínua sobre $\bar{B} \backslash\{0\}$ que pertencente à classe de equivalência de $u(U \sim u$ se $U=u$ q.t.p). Neste texto todo elemento $u \in$ $W_{\text {rad }}^{1, p}(B)$ estará representado por seu representante contínuo $U$ como acima. Também para simplificar a notação, $u$ denotará seu representante contínuo.

No que segue deste capítulo, o espaço $H_{0}^{1}(B)$ estará munido com a norma:

$$
\|u\|=\left(\int_{B}|\nabla u|^{2} d x\right)^{1 / 2}, \quad \forall u \in H_{0}^{1}(B) .
$$

Denota-se $H_{0, \text { rad }}^{1}(B)=\left\{u \in H_{0}^{1}(B)\right.$ tal que $\left.u \circ O=u, \forall O \in O(N)\right\}$.

Teorema 2.7. Seja $N \geq 3$. Então existe uma constante positiva $C$ tal que para toda $u \in$ $H_{0, \mathrm{rad}}^{1}(B)$,

$$
|u(x)| \leq C \frac{\|u\|}{|x|^{\frac{N-2}{2}}}, \quad \forall x \in \bar{B} \backslash\{0\} .
$$

Proposição 2.8. (Interpolação) Seja $(\Omega, \Sigma, \mu)$ um espaço de medida e E um espaco de Banach reflexivo. Suponha que para $1 \leq p<q \leq \infty$, a imersão $E \hookrightarrow L^{p}(\Omega)$ é compacta e a imersão 
$E \hookrightarrow L^{q}(\Omega)$ é contínua. Então para todo $r$ tal que $p<r<q$, a imersão $E \hookrightarrow L^{r}(\Omega)$ é compacta.

Demonstração. Seja $p<r<q$, então $r=\theta p+(1-\theta) q$ para algum $\theta \in(0,1)$. Seja $u_{n} \rightarrow u$ em $E$, note que pela imersão compacta de $E$ em $L^{p}(\Omega)$ tem-se $u_{n} \longrightarrow u$ em $L^{p}(\Omega)$. Também observe que $\left(u_{n}\right)$ é limitado em $E$ e a partir da imersão continua de $E$ em $L^{q}(\Omega)$ tem-se $\left(u_{n}\right)$ é limitado em $L^{q}(\Omega)$. Assim, $\left(u_{n}-u\right)$ é limitado em $L^{q}(\Omega)$. No que segue mostra-se que $u_{n} \longrightarrow u$ em $L^{r}(\Omega)$, de fato

$$
\int_{B}\left|u_{n}-u\right|^{r} d \mu=\int_{B}\left|u_{n}-u\right|^{\theta p+(1-\theta) q} d \mu=\int_{B}\left|u_{n}-u\right|^{\theta p}\left|u_{n}-u\right|^{(1-\theta) q} d \mu .
$$

Aplicando a desigualdade de Hölder (Teorema A.2) com expoentes conjugados $\theta$ e $1-\theta$, resulta

$$
\begin{aligned}
\int_{B}\left|u_{n}-u\right|^{r} d \mu & \leq\left(\int_{B}\left|u_{n}-u\right|^{p}\right)^{\theta}\left(\int_{B}\left|u_{n}-u\right|^{q}\right)^{1-\theta} d \mu \\
& =\left|u_{n}-u\right|_{p}^{p \theta}\left|u_{n}-u\right|_{q}^{q(1-\theta)} d \mu \longrightarrow 0 .
\end{aligned}
$$

Assim, $\left|u_{n}-u\right|_{r}^{r} \longrightarrow 0$ o que implica que $u_{n} \longrightarrow u$ em $L^{r}(\Omega)$. Portanto a proposição está provada.

Para $p \geq 1$ e $\alpha>0$. Denote por $L^{p}\left(B,|x|^{\beta}\right)$ ao espaço de funções com respeito à medida $|x|^{\alpha} d x$, i.e.,

$$
L^{p}\left(B,|x|^{\alpha}\right)=\left\{u: B \rightarrow \mathbb{R} \text { mensuravel; } \int_{B}|u(x)|^{p}|x|^{\alpha} d x<\infty\right\} .
$$

Dotado com a norma $|u|_{p, \alpha}=\left(\int_{B}|x|^{\alpha}|u(x)|^{p} d x\right)^{1 / p}$.

Proposição 2.9. [12, Corollary 1.2] Sejam $N \geq 3 e \alpha>0$. Então,

(i) A imersão $H_{0, \mathrm{rad}}^{1}(B) \hookrightarrow L^{p}\left(B,|x|^{\alpha}\right)$ é continua para todo $1 \leq p \leq 2_{\alpha^{\prime}}^{*}$

(ii) A imersão $H_{0, \mathrm{rad}}^{1}(B) \hookrightarrow L^{p}\left(B,|x|^{\alpha}\right)$ é compacta para $1 \leq p<2_{\alpha}^{*}$.

Demonstração. Para provar o item $(i)$, uma vez que $|x|^{\alpha} d x$ é uma medida finita sobre $B$. Pela proposição B.3, basta mostrar que a imersão

$$
H_{0, \operatorname{rad}}^{1}(B) \hookrightarrow L^{2_{\alpha}^{*}}\left(B,|x|^{\alpha}\right) \text { es continua, }
$$


isto é, $|u|_{2_{\alpha}^{*}} \leq C\|u\|$ para todo $u \in H_{0 \text {,rad }}^{1}(B)$. Seja $u \in H_{0, \text { rad }}^{1}(B)$, então

$$
\int_{B}|u|^{2_{\alpha}^{*}}|x|^{\alpha} d x=\int_{B}|u|^{\frac{2 N+2 \alpha}{N-2}}|x|^{\alpha} d x=\int_{B}|u|^{\frac{2 N}{N-2}}|u|^{\frac{2 \alpha}{N-2}}|x|^{\alpha} d x
$$

Pelo Teorema 2.7, existe $C>0$ tal que

$$
|u|^{\frac{2 \alpha}{N-2}}|x|^{\alpha} \leq C^{\frac{2 \alpha}{N-2}}\|u\|^{\frac{2 \alpha}{N-2}}|x|^{-\frac{2 \alpha}{N-2} \frac{(N-2)}{2}}|x|^{\alpha} \leq C^{\frac{2 \alpha}{N-2}}\|u\|^{\frac{2 \alpha}{N-2}}, \quad \forall x \in \bar{B} \backslash\{0\} .
$$

Assim, de (2.8)-(2.9) e pela imersão de Sobolev $H_{0, \text { rad }}^{1}(B) \hookrightarrow L^{2^{*}}(B)$, tem-se

$$
\int_{B}|u|^{2_{\alpha}^{*}}|x|^{\alpha} d x \leq C^{\frac{2 \alpha}{N-2}}\|u\|^{\frac{2 \alpha}{N-2}}\|u\|_{2^{*}}^{2^{*}} \leq C^{\frac{2 \alpha}{N-2}}\|u\|^{\frac{2 \alpha}{N-2}} C_{1}^{2^{*}}\|u\|^{2^{*}}=C_{2}\|u\|^{2_{\alpha}^{*}}
$$

onde $C_{2}=C^{\frac{2 \alpha}{N-2}} C_{1}^{2^{*}}$, para algum $C_{1}>0$. Portanto

$$
\|u\|_{2_{\alpha}^{*}} \leq C_{2}^{1 / 2_{\alpha}^{*}}\|u\|
$$

Para provar o item $(i i)$, observe que a imersão $H_{0}^{1}(B)$ em $L^{1}(B)$ é compacta e a imersão $L^{1}(B)$ em $L^{1}\left(B,|x|^{\alpha}\right)$ é contínua, então disto obtém-se que a composição é compacta, i.e., a imersão $H_{0 \text {,rad }}^{1}(B) \hookrightarrow L^{1}\left(B,|x|^{\alpha}\right)$ é compacta. Também note que pelo item $(i)$, tem-se que a imersão $H_{0, \text { rad }}^{1}(B)$ em $L^{2_{\alpha}^{*}}\left(B,|x|^{\alpha}\right)$ é contínua. Portanto usando a Proposição 2.8 para $1 \leq p<2_{\alpha}^{*}$ é imediato provar que a imersão $H_{0, \text { rad }}^{1}(B) \hookrightarrow L^{p}\left(B,|x|^{\alpha}\right)$ é compacta.

\subsection{Existência de solução}

Nesta seção é mostrado que o problema (2.1) tem solução positiva no caso em que $1<p<2^{*}$ e $p \neq 2$. Mesmo que implicitamente, estaremos supondo a condição $1<p<$ $2^{*}$ e $p \neq 2$. O procedimento para encontrar tal solução é o de minimizar o funcional $J: H_{0}^{1}(B) \rightarrow \mathbb{R}$, definido por

$$
J(u)=\frac{1}{2} \int_{B}|\nabla u|^{2} d x-\frac{1}{p} \int_{B}|x|^{\alpha}|u|^{p} d x, \quad u \in H_{0}^{1}(B)
$$

em sua variedade de Nehari. 
Este funcional está bem definido em virtude do Lema B.7 com $J \in C^{1}\left(H_{0}^{1}(B), \mathbb{R}\right)$ e

$$
J^{\prime}(u) v=\int_{B} \nabla u \cdot \nabla v d x-\int_{B}|x|^{\alpha}|u|^{p-2} u v d x, \quad \forall u, v \in H_{0}^{1}(B) .
$$

Definição 2.10. Suponha $1<p<2^{*}$ e $p \neq 2$. Diz-se que u é solução fraca de (2.1) se ué ponto critico do funcional $J$, isto é, se $u \in H_{0}^{1}(B)$ e satisfaz

$$
\int_{B} \nabla u \cdot \nabla v d x=\int_{B}|x|^{\alpha}|u|^{p-2} u v d x, \quad \forall v \in H_{0}^{1}(B)
$$

Mais adiante, na Seção 2.3, fazendo uso de resultados de regularidade, vai ser mostrado que qualquer solução fraca de (2.1) como na Definição 2.10 é na verdade solução clássica para o problema (2.1).

Definição 2.11. (solução de energia mínima) Suponha $1<p<2^{*}$ e $p \neq 2$. Diz-se que ué solução ground state para (2.1) se u é solução fraca não trivial de (2.1) e

$$
J(u)=\min \{J(v) ; v \text { é solução fraca não trivial de } 2.1]\} .
$$

Suponha $1<p<2^{*}, p \neq 2$. Denota-se por $N_{J}$ a variedade Nehari associada ao funcional $J$, a saber

$$
N_{J}:=\left\{u \in H_{0}^{1}(B) ; J^{\prime}(u) u=0, u \neq 0\right\} .
$$

Considere os problemas de minimização

$$
c_{J}:=\inf _{u \in N_{J}} J(u)
$$

e

$$
S_{\alpha, p}:=\inf _{\substack{u \in H_{0}^{1}(B) \\ u \neq 0}} \frac{\int_{B}|\nabla u|^{2} d x}{\left(\int_{B}|x|^{\alpha}|u|^{p} d x\right)^{2 / p}} .
$$

Observe que (veja Proposição B.1)

$$
\inf _{\substack{u \in H_{0}^{1}(B) \\ u \neq 0}} \frac{\int_{B}|\nabla u|^{2} d x}{\left(\int_{B}|x|^{\alpha}|u|^{p} d x\right)^{2 / p}}=\inf _{u \in H_{0}^{1}(B)}\left\{\int_{B}|\nabla u|^{2} d x,|u|_{p, \alpha}^{p}=1\right\} .
$$

Então

$$
S_{\alpha, p}=\inf _{u \in H_{0}^{1}(B)}\left\{\int_{B}|\nabla u|^{2} d x,|u|_{p, \alpha}^{p}=1\right\} .
$$

Lema 2.12. [28, Lemma 4.1] Para qualquer $u \in H_{0}^{1}(B) \backslash\{0\}$ existe um único $t=t(u)>0$ tal 
que tu $\in N_{J}$. Além disso, o máximo de $J(t u)$ quando $t \geq 0$ é atingido em $t=t(u)$. A função

$$
\begin{aligned}
\phi: H_{0}^{1}(B) \backslash\{0\} & \longrightarrow(0, \infty) \\
u & \longmapsto \phi(u)=t(u) .
\end{aligned}
$$

é continua e a aplicação $u \mapsto t(u) u$ define um homeomorfismo da esfera unitária de $H_{0}^{1}(B)$ com $N_{J}$.

Demonstração. Dado $u \in H_{0}^{1}(B) \backslash\{0\}$ considere a função

$$
\begin{aligned}
g_{u}:[0, \infty) & \longrightarrow \mathbb{R} \\
t & \longmapsto g_{u}(t)=J(t u) .
\end{aligned}
$$

Para $t>0$, tem-se que $g_{u}^{\prime}(t)=0$ se, e somente se $t u \in N_{J}$. Além disso, observe que

$$
\begin{aligned}
g_{u}^{\prime}(t) & =J^{\prime}(t u) u=t \int_{B}|\nabla u|^{2} d x-\int_{B}|x|^{\alpha}|t u|^{p-2} t u u d x \\
& =t \int_{B}|\nabla u|^{2} d x-t^{p-1} \int_{B}|x|^{\alpha}|u|^{p} d x \\
& =t\left(\int_{B}|\nabla u|^{2} d x-t^{p-2} \int_{B}|x|^{\alpha}|u|^{p} d x\right) .
\end{aligned}
$$

Assim, existe único $t>0$ tal que $g_{u}^{\prime}(t)=0$. Este número vai ser denotado por $t(u) \mathrm{e}$ observe que

$$
t(u)=\left(\frac{\|u\|^{2}}{|u|_{p, \alpha}^{p}}\right)^{\frac{1}{p-2}} .
$$

Além disso, $g_{u}^{\prime}(t)>0$ se $t \in(0, t(u))$ e $g_{u}^{\prime}(t)<0$ se $t>t(u)$. Logo tem-se que $\max _{t \geq 0} g(t)$ é atingido por um único $t(u)$, isto é,

$$
\max _{t \geq 0} J(t u)=J(t(u) u)
$$

Para provar a continuidade de $\phi$, assuma que $u_{n} \longrightarrow u$ em $H_{0}^{1}(B) \backslash\{0\}$. Da continuidade da norma, tem-se então que $\|u\|^{2} \longrightarrow\|u\|^{2}$ quando $n \longrightarrow \infty$. Por outro lado, aplicando o Teorema de Rellich-Kondrachov (Teorema A.20 implica que $u_{n} \longrightarrow u$ em $L^{p}(B)$ e usando Teorema A.9, obtém-se as condições necessárias para aplicar o Teorema da Convergência Dominada (Teorema A.8). Assim, resulta que $\left|u_{n}\right|_{p, \alpha} \longrightarrow|u|_{p, \alpha}$ quando $n \longrightarrow \infty$. Logo tendo em vista a identidade (2.12) conlui-se $t\left(u_{n}\right) \longrightarrow t(u)$ quando $n \longrightarrow \infty$. Portanto $\phi$ é continua.

Finalmente para provar o homeomorfismo entre $S_{H_{0}^{1}(B)}=\left\{u \in H_{0}^{1}(B) ;\|u\|=1\right\}$ (a 
esfera unitária de $\left.H_{0}^{1}(B)\right)$ e $N_{J}$. Seja

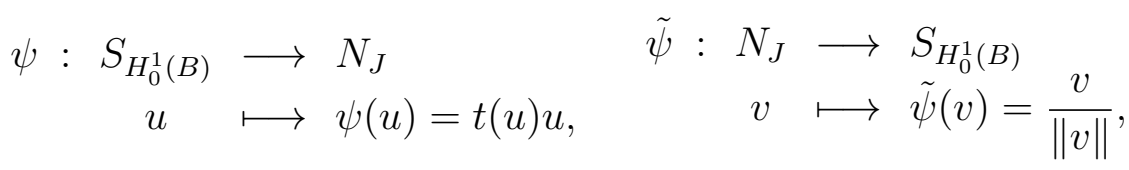

é imediato ver que as aplicações $\psi$ e $\tilde{\psi}$ são continuas. Para mostrar a injetividade de $\psi$, seja $u, v \in B$. Se $\psi(u)=\psi(v)$ então $t(u) u=t(v) v$ e $|t(u)|\|u\|=|t(v)|\|v\|$. Como $t(u), t(v)$ são positivas, então $t(u)=t(v)$ assim $u=v$.

Para mostrar a sobrejetividade de $\psi$, tome $v \in N_{J}$ e considere $u=\frac{v}{\|v\|} \in B$, note que $\psi(u)=t\left(\frac{v}{\|v\|}\right) \frac{v}{\|v\|}=\|v\| \frac{v}{\|v\|}=v$. Portanto $\psi$ é bijeção.

Deste lema observe que

$$
t u \in N_{J} \operatorname{com} t>0 \Longleftrightarrow t=t(u)=\left(\frac{\|u\|^{2}}{|u|_{p, \alpha}^{p}}\right)^{\frac{1}{p-2}} .
$$

Por outro lado, note que se $u \in N_{J}$ então $u \neq 0$ e

$$
0=J^{\prime}(u) u=\|u\|^{2}-\int_{B}|x|^{\alpha}|u|^{p} d x=\|u\|^{2}-|u|_{p, \alpha}^{p}
$$

Assim

$$
\|u\|^{2}=|u|_{p, \alpha}^{p}
$$

Isto implica em

$$
J(u)=\frac{1}{2}\|u\|^{2}-\frac{1}{p}|u|_{p, \alpha}^{p}=\frac{p-2}{2 p}\|u\|^{2}
$$

usando isto resulta,

$$
\frac{\|u\|^{2}}{|u|_{p, \alpha}^{2}}=\frac{\|u\|^{2}}{\left(|u|_{p, \alpha}^{p}\right)^{\frac{2}{p}}}=\frac{\|u\|^{2}}{\left(\|u\|^{2}\right)^{\frac{2}{p}}}=\left(\|u\|^{2}\right)^{1-\frac{2}{p}}=\left(\frac{2 p}{p-2} J(u)\right)^{\frac{p-2}{p}} .
$$

Observação 2.13. Note que se $1<p<2, J(u)$ é negativo e assim a última identidade tem sentido.

Lema 2.14. [6. Lemma 2.3] Suponha $1<p<2^{*}$ e $p \neq 2$. Os problemas de minimização (2.10) $e$ (2.11) são equivalentes no seguinte sentido:

(i) Dada uma sequência minimizante $\left(u_{n}\right) \subseteq N_{J}$ para $(2.10),\left(\left|u_{n}\right|_{p, \alpha}^{-1} u_{n}\right)$ é sequência minimizante para (2.11). 
(ii) Dada uma sequência minimizante $\left(u_{n}\right)$ para 2.11$),\left(\left\|\bar{u}_{n}\right\|^{\frac{2}{p-2}} \bar{u}_{n}\right) \subseteq N_{J}$ é sequência minimizante para (2.10).

(iii) Tem-se a igualdade $c_{J}=\frac{p-2}{2 p} S_{\alpha, p}^{\frac{p}{p-2}}$.

(iv) A constante de Sobolev $S_{\alpha, p}$ é atingida se, e somente se, $c_{J}$ é atingido.

\section{Demonstração.}

i) Seja $\left(u_{n}\right) \subseteq N_{J}$ sequência minimizante para (2.10). Então $\lim _{n \rightarrow \infty} J\left(u_{n}\right)=c_{J}$ e da estimativa 2.11 , tem-se que $S_{\alpha, p} \leq\left\|\left|u_{n}\right|_{p, \alpha}^{-1} u_{n}\right\|^{2}=\frac{\left\|u_{n}\right\|^{2}}{\left|u_{n}\right|_{p, \alpha}^{2}}$. A partir da estimativa 2.16, obtém-se

$$
S_{\alpha, p} \leq \lim _{n \rightarrow \infty} \frac{\left\|u_{n}\right\|^{2}}{\left|u_{n}\right|_{p, \alpha}^{2}}=\lim _{n \rightarrow \infty}\left(\frac{2 p}{p-2} J\left(u_{n}\right)\right)^{\frac{p-2}{p}}=\left(\frac{2 p}{p-2} c_{J}\right)^{\frac{p-2}{p}} .
$$

Por outro lado, seja $u \in H_{0}^{1}(B) \backslash\{0\} \quad$ com $|u|_{p, \alpha}=1$, pelo Lema 2.12, existirá um único $t=t(u)$ tal que $t u \in N_{J}$. Logo da estimativa 2.14 considerando $t u$, resulta

$$
\left(\frac{2 p}{p-2} c_{J}\right)^{\frac{p-2}{p}} \leq\left(\frac{2 p}{p-2} J(t u)\right)^{\frac{p-2}{p}}=\frac{\|t u\|^{2}}{|t u|_{p, \alpha}^{2}}=\frac{\|u\|^{2}}{|u|_{p, \alpha}^{2}}=\|u\|^{2}, \quad \forall u \in H_{0}^{1}(B) .
$$

Então,

$$
\left(\frac{2 p}{p-2} c_{J}\right)^{\frac{p-2}{p}} \leq S_{\alpha, p}
$$

As expressões (2.17) e (2.18), acarretam em

$$
S_{\alpha, p}=\lim _{n \rightarrow \infty} \frac{\left\|u_{n}\right\|^{2}}{\left|u_{n}\right|_{p, \alpha}^{2}}=\lim _{n \rightarrow \infty}\left\|\left|u_{n}\right|_{p, \alpha}^{-1} u_{n}\right\|^{2} .
$$

ii) Seja $\left(\bar{u}_{n}\right)$ sequência minimizante para (2.11). Então $\bar{u}_{n} \in H_{0}^{1}(B),\left|\bar{u}_{n}\right|_{p, \alpha}=1 \mathrm{e}$ $S_{\alpha, p}=\lim _{n \rightarrow \infty}\left\|\bar{u}_{n}\right\|^{2}$. Pelo Lema 2.12 para cada $n \in \mathbb{N}$ fixo arbitrario, existe $t=t\left(u_{n}\right)$ tal que

$$
t=\left(\frac{\left\|\bar{u}_{n}\right\|^{2}}{\left|\bar{u}_{n}\right|_{p, \alpha}^{p}}\right)^{\frac{1}{p-2}}=\left\|\bar{u}_{n}\right\|^{\frac{2}{p-2}} \quad \text { e } \quad\left\|\bar{u}_{n}\right\|^{\frac{2}{p-2}} \bar{u}_{n} \in N_{J} .
$$

Logo para $\left\|\bar{u}_{n}\right\|^{\frac{2}{p-2}} \bar{u}_{n}$ na estimativa 2.15 , segue-se

$$
c_{J} \leq J\left(\left\|\bar{u}_{n}\right\|^{\frac{2}{p-2}} \bar{u}_{n}\right)=\left(\frac{p-2}{2 p}\right)\left\|\bar{u}_{n}\right\|^{\frac{4}{p-2}}\left\|\bar{u}_{n}\right\|^{2}=\left(\frac{p-2}{2 p}\right)\left\|\bar{u}_{n}\right\|^{\frac{2 p}{p-2}} .
$$


Daí,

$$
c_{J} \leq \lim _{n \rightarrow \infty} J\left(\left\|\bar{u}_{n}\right\|^{\frac{2}{p-2}} \bar{u}_{n}\right)=\frac{p-2}{2 p} \lim _{n \rightarrow \infty}\left(\left\|\bar{u}_{n}\right\|^{2}\right)^{\frac{p}{p-2}}=\frac{p-2}{2 p} S_{\alpha, p}^{\frac{p}{p-2}}
$$

Por outro lado, seja $u \in N_{J}$ pela definição de $S_{\alpha, p}$ e a estimativa (2.14), obtém-se

$$
S_{\alpha, p} \leq \frac{\|u\|^{2}}{\left(|u|_{p, \alpha}^{p}\right)^{\frac{2}{p}}}=\frac{\|u\|^{2}}{\|u\|^{\frac{4}{p}}}=\|u\|^{\frac{2 p-4}{p}} .
$$

Agora fazendo uso da estimativa (2.15), resulta

$$
\frac{p-2}{2 p} S_{\alpha, p}^{\frac{p}{p-2}} \leq \frac{p-2}{2 p}\left(\|u\|^{\frac{2 p-4}{p}}\right)^{\frac{p}{p-2}}=\frac{p-2}{2 p}\|u\|^{2}=J(u), \quad \forall u \in N_{J} .
$$

Assim,

$$
\frac{p-2}{2 p} S_{\alpha, p}^{\frac{p}{p-2}} \leq c_{J}
$$

Logo as desigualdades 2.19 e 2.20 acarretam em $c_{J}=\lim _{n \rightarrow \infty} J\left(\left\|u_{n}\right\|^{\frac{2}{p-2}} u_{n}\right)$.

iii) Da definição de ínfimo em (2.11) existe uma sequência $\left(u_{n}\right) \subseteq H_{0}^{1}(B) \backslash\{0\}$ com $\left|u_{n}\right|_{p, \alpha}^{p}=1$ tal que

$$
\left\|u_{n}\right\|^{2} \longrightarrow S_{\alpha, p}, \quad \text { quando } n \longrightarrow \infty
$$

Logo do item $(i i)$ segue-se que $c_{J}=\lim _{n \rightarrow \infty} J\left(\left\|\bar{u}_{n}\right\|^{\frac{2}{p-2}} \bar{u}_{n}\right)$ com $\left\|\bar{u}_{n}\right\|^{\frac{2}{p-2}} \bar{u}_{n} \in N_{J}$. Assim, tendo en vista a estimativa (2.15) implica

$$
\begin{aligned}
J\left(\left\|u_{n}\right\|^{\frac{2}{p-2}} u_{n}\right) & =\frac{p-2}{2 p}\|\| u_{n}\left\|^{\frac{2}{p-2}} u_{n}\right\|^{2}=\frac{p-2}{2 p}\left\|u_{n}\right\|^{\frac{4}{p-2}}\left\|u_{n}\right\|^{2}=\frac{p-2}{2 p}\left\|u_{n}\right\|^{\frac{2 p}{p-2}} \\
& =\frac{p-2}{2 p}\left(\left\|u_{n}\right\|^{2}\right)^{\frac{p}{p-2}}
\end{aligned}
$$

Fazendo $n \longrightarrow \infty$ e da estimativa 2.21 , obtém-se $J\left(\left\|u_{n}\right\|^{\frac{2}{p-2}} u_{n}\right) \longrightarrow \frac{p-2}{2 p} S_{\alpha, p}^{\frac{p}{p-2}}$. Portanto,

$$
c_{J}=\frac{p-2}{2 p} S_{\alpha, p}
$$

iv) Suponha que $S_{\alpha, p}$ é atingida, isto é existe $\bar{u} \in H_{0}^{1}(B) \operatorname{com}|\bar{u}|_{p, \alpha}=1$ e $S_{\alpha, p}=\|\bar{u}\|^{2}$. Do Lema 2.12 e da expressão 2.13) tem-se $\|\bar{u}\|^{\frac{2}{p-2}} \bar{u} \in N_{J}$ ou ainda $S_{\alpha, p}^{\frac{1}{p-2}} \bar{u} \in N_{J}$. Por outro lado note que para todo $u \in N_{J}$ e usando a estimativa (2.16) tem-se

$$
S_{\alpha, p} \leq \frac{\|u\|^{2}}{|u|_{p, \alpha}^{2}}=\left(\frac{2 p}{p-2} J(u)\right)^{\frac{p-2}{p}}
$$


Também

$$
\left(\frac{2 p}{p-2} J\left(S_{\alpha, p}^{\frac{1}{p-2}} \bar{u}\right)\right)^{\frac{p-2}{p}}=\frac{S_{\alpha, p}^{\frac{2}{p-2}}\|\bar{u}\|^{2}}{S_{\alpha, p}^{\frac{2}{p-2}}|\bar{u}|_{p, \alpha}^{2}}=\|\bar{u}\|^{2}=S_{\alpha, p} .
$$

Logo das expressões 2.22 e 2.23 conclui-se que $J\left(S_{\alpha, p}^{\frac{1}{p-2}} \bar{u}\right) \leq J(\bar{u})$ para todo $u \in N_{J}$. Assim $\left.J\left(S_{\alpha, p}^{\frac{1}{p-2}} \bar{u}\right)\right) \leq c_{J}$. Portanto $\left.c_{J}=J\left(S_{\alpha, p}^{\frac{1}{p-2}} u\right)\right)$.

Reciprocamente, suponha que $c_{J}$ é atingido, então existe $u \in N_{J}$ tal que $J(u)=c_{J}$. Logo pela estimativa (2.16) e do item (iii), obtém-se

$$
\frac{\|u\|^{2}}{\left(\int_{B}|x|^{\alpha}|u|^{p} d x\right)^{2 / p}}=\left(\frac{2 p}{p-2} J(u)\right)^{\frac{p-2}{p}}=\left(\frac{2 p}{p-2} C_{J}\right)^{\frac{p-2}{p}}=S_{\alpha, p} .
$$

Então u é minimizante para (2.11).

Observação 2.15. Note que se $S_{\alpha, p}$ é atingido por $u$, então $c_{J}$ é atingido por $\|u\|^{\frac{2}{p-2}} u \in N_{J}$. Reciprocamente, se $c_{J}$ é atingido por u então $S_{\alpha, p}$ é atingido por $|u|_{p, \alpha}^{-1} u$.

Aqui e no que segue denotamos por $|u|_{p}$ a $L^{p}$-norma da função $u \in L^{p}(B)$.

Proposição 2.16. [6. Lema 2.5] Suponha $1<p<2^{*}$ e $p \neq 2$. Se $u \in N_{J}$ é tal que $J(u)=c_{J}$, então u é solução ground state para (2.1). Reciprocamente, se u é solução ground state para (2.1), então $J(u)=c_{J}$.

Demonstração. Seja

$$
\begin{aligned}
G: H_{0}^{1}(B) & \longrightarrow \mathbb{R} \\
u & \longmapsto G(u)=J^{\prime}(u) u=\|u\|^{2}-\int_{B}|x|^{\alpha}|u|^{p} d x .
\end{aligned}
$$

Define-se

$$
\begin{aligned}
\phi: H_{0}^{1}(B) & \longrightarrow \mathbb{R} \\
u & \longmapsto \phi(u)=\|u\|^{2} . \\
\psi: H_{0}^{1}(B) & \longrightarrow \mathbb{R} \\
u & \longmapsto \psi(u)=\int_{B}|x|^{\alpha}|u|^{p} d x .
\end{aligned}
$$

Procedendo de forma similar como foi feito no Lema B.7, obtém-se

$$
\begin{gathered}
\phi^{\prime}(u) v=2\langle u, v\rangle=2 \int_{B} \nabla u \cdot \nabla v d x, \quad \forall u, v \in H_{0}^{1}(B) . \\
\psi^{\prime}(u) v=\int_{B} p|x|^{\alpha} u|u|^{p-2} v d x .
\end{gathered}
$$


Desta duas ultimas igualdades resulta

$$
G^{\prime}(u) v=2\langle u, v\rangle-p \int_{B}|x|^{\alpha} u|u|^{p-2} v d x, \quad \forall u, v \in H_{0}^{1}(B) .
$$

Em particular fazendo $v=u$, tem-se

$$
G^{\prime}(u) u=2\|u\|^{2}-p \int_{B}|x|^{\alpha}|u|^{p} d x, \quad \forall u \in H_{0}^{1}(B) .
$$

Usando (2.14), resulta $G^{\prime}(u) u=(2-p)\|u\|^{2} \neq 0$ para todo $u \in N_{J}$. Portanto

$$
G^{\prime}(u) \neq 0, \quad \forall u \in N_{J}
$$

Agora seja $u \in N_{J}$ tal que $J(u)=\min _{u \in N_{J}} J(u)$, isto juntamente com 2.25) permite aplicar o Teorema dos Multiplicadores de Lagrange (ver Teorema A.5) o qual vai fornecer um número $\lambda \in \mathbb{R}$ tal que $J^{\prime}(u)=\lambda G^{\prime}(u)$. De isto,

$$
0=J^{\prime}(u) u=\lambda G^{\prime}(u) u
$$

Como $G^{\prime}(u) u \neq 0$, então $\lambda=0$. Logo $u$ é ponto critico não trivial de $J$. Assim, u é solução para o problema (2.1).

Por outro lado, seja $v$ ponto critico não trivial de $J$, então $v \in N_{J}$ e pela hipótese resulta $J(u) \leq J(v)$. Também se $c \leq J(v)$ para todo ponto critico $v$ não trivial de $J$, desde que $u$ é também ponto critico, tem-se $J(u)$ é a maior das cotas inferiores. Assim

$$
J(u)=\min \{J(v) ; v \text { é ponto critico não trivial de } J\} \text {. }
$$

Portanto, $u$ é solução de energia minima para (2.1).

Reciprocamente, seja $u$ solução ground state para o problema (2.1) da Proposição 2.17 tem-se que $S_{\alpha, p}$ é atingido. Suponha que seja atingido por $\bar{u}$, então da observação 2.15. diz-se que $\tilde{u}=\|\bar{u}\|^{\frac{2}{p-2}} \bar{u} \in N_{J}$ e $c_{J}=J(\tilde{u})$, com isto e usando a primeira parte do teorema mesmo tem-se que $\tilde{u}$ é solução ground state para (2.1).

Logo, desde que $u$ e $\tilde{u}$ são soluções ground state para (2.1), então $J(u)=J(\tilde{u})$. Portanto $c_{J}=J(u)$.

Na seguinte proposição mostra-se que sempre existe um minimizador $u$ para o problema de minimização (2.11), ou seja existe uma função não trivial $u \in H_{0}^{1}(B)$ com $|u|_{p}=1$ tal que $S_{\alpha, p}=\|u\|^{2}$. 
Proposição 2.17. [6. Lema 2.4] Suponha $1<p<2^{*}$ e $p \neq 2$ e $\alpha \geq 0$. Então a constante de Sobolev $S_{\alpha, p}$ é atingida. Além disso, se $S_{\alpha, p}$ é atingido por $u$, então u é $C^{2}(\bar{B})$ e $|u|>0$ em $B$, isto é, $u>0 \mathrm{em} \mathrm{B}$ ou $u<0 \mathrm{em} B$.

Demonstração. Sejam:

$$
\begin{aligned}
I: H_{0}^{1}(B) & \longrightarrow \mathbb{R} \\
u & \longmapsto J(u)=\|u\|^{2},
\end{aligned}
$$

e

$$
M=\left\{u \in H_{0}^{1}(B) ; \int_{B}|x|^{\alpha}|u|^{p} d x=1\right\}
$$

Considerando $A=\{I(u), u \in M\} \subseteq \mathbb{R}$ conjunto não vazio e limitado inferiormente, isto fornece que existe $\lambda \in \mathbb{R}$ tal que $\lambda=\inf _{u \in M} I(u)$. Segue-se da definição de ínfimo que existe uma sequência $\left(u_{n}\right) \subseteq M$ tal que

$$
I\left(u_{n}\right) \longrightarrow \lambda \text { quando } n \longrightarrow \infty \text {. }
$$

Assim, a sequência $\left(u_{n}\right)$ é limitada em $H_{0}^{1}(B)$. Agora desde que $H_{0}^{1}(B)$ é um espaço reflexivo, então pelo Teorema de Kakutani (ver Teorema A.7), existe $u_{0} \in H_{0}^{1}(B)$ tal que ao menos de subsequência

$$
u_{n} \rightarrow u_{0} \quad \text { em } H_{0}^{1}(B)
$$

Além disso,

$$
\left\|u_{0}\right\|^{2} \leq \liminf _{n \rightarrow \infty}\left\|u_{n}\right\|^{2}
$$

Afirmação: $u_{0} \in M$.

De fato, pela estimativa (2.28) e Teorema de Rellich- Kondrachov, isto é Teorema A.20, tem-se $u_{n} \longrightarrow u_{0}$ em $L^{p}(B)$ isto juntamente com o Teorema A.9 fornece uma subsequência $\left(u_{n_{i}}\right) \subseteq\left(u_{n}\right)$ e uma função $h \in L^{p}(B)$ tal que:

(i) $|x|^{\alpha} u_{n_{i}}(x) \longrightarrow|x|^{\alpha} u_{0}(x)$ q.t.p. em B,

(ii) $|x|^{\alpha} u_{n_{i}}(x) \leq|x|^{\alpha} h(x) \quad$ q.t.p. em B,

onde $|x|^{\alpha} h \in L^{p}(B)$. Assim pelo Teorema da Convergência Dominada de Lebesgue (ver Teorema A.8) obtém-se

$$
\lim _{i \rightarrow \infty} \int_{B}|x|^{\alpha}\left|u_{n_{i}}\right|^{p} d x=\int_{B}|x|^{\alpha}\left|u_{0}\right|^{p} d x
$$


Como $u_{n_{i}} \in M$ para todo $i$, então $\int_{B}|x|^{\alpha}\left|u_{n_{i}}\right|^{p} d x=1$. Assim $\int_{B}|x|^{\alpha}\left|u_{0}\right|^{p} d x=1$. Portanto $u_{0} \in M$ e afirmação está provada.

Logo desta afirmação e das expressões (2.26)-(2.29)-(2.27) resulta

$$
\lambda \leq I\left(u_{0}\right)=\left\|u_{0}\right\|^{2} \leq \liminf _{i \rightarrow \infty}\left\|u_{n_{i}}\right\|^{2}=\lambda .
$$

Tendo assim ,

$$
I\left(u_{0}\right)=\lambda, \quad(\lambda \geq 0)
$$

Afirmação: $\lambda>0$.

De fato, suponha por absurdo que $\lambda=0$. Assim de (2.27), obtem-se $I\left(u_{n}\right) \longrightarrow 0$ quando $n \rightarrow \infty$, o que implica que $u_{n} \longrightarrow 0$ em $H_{0}^{1}(B)$. Pelo Teorema de RellichKondrachov tem-se $u_{n} \longrightarrow 0$ em $L^{p}(B)$ quando $n \longrightarrow \infty$. Daí,

$$
\left.\left.\left|\int_{B}\right| x\right|^{\alpha}\left|u_{n}\right|^{p} d x\left|\leq \int_{B}\right| u_{n}\right|^{p} d x=\left|u_{n}\right|_{p}^{p} \longrightarrow 0
$$

Logo para $\varepsilon=1$, existe $n_{0} \in \mathbb{N}$ tal que $\int_{B}|x|^{\alpha}\left|u_{n_{0}}\right|^{p} d x<1$. Isto é uma contradição, pois $u_{n_{0}} \in M$. Portanto $\lambda>0$.

Agora tendo em vista que $u_{0} \in M$ e as expressões (2.11)-(2.30), conclui-se

$$
S_{\alpha, p}=\inf _{u \in H_{0}^{1}(B)}\left\{\|u\|^{2} d x,|u|_{p, \alpha}^{p}=1\right\}=\inf _{u \in M} I(u)=I\left(u_{0}\right) .
$$

Assim $S_{\alpha, p}$ é atingido por $u_{0}$. Além disso, visto que $\lambda>0$ e $I\left(u_{0}\right)=\lambda$, então segue que $u_{0} \neq 0$ e também tem-se (ver Proposição B.2),

$$
\int_{B}\left|\nabla u_{0}\right|^{2} d x=\int_{B}|\nabla| u_{0}||^{2} d x \quad e \quad 1=\left|u_{0}\right|_{p, \alpha}=|| u_{0}||_{p, \alpha} .
$$

Assim, diz-se também que $\left|u_{0}\right|$ atinge a $S_{\alpha, p}$. A partir da Observação 2.15 tem-se que existe $\lambda>0$ tal que $v=\lambda\left|u_{0}\right| \in N_{J}$ e $c_{J}=J(v)$. Aplicando o Teorema 2.16 tem-se $v$ é solução para o problema (2.1). Logo $v$ satisfaz

$$
-\Delta v=|x|^{\alpha}|v|^{p-2} v, \quad \text { em } B, \quad v=0 \text { sobre } \partial B .
$$

Usando a teoria de regularização, (ver Teorema 2.20 da próxima seção) tem-se que $u \in C^{2}(\bar{B})$. Assim, pelo Principio do Máximo Forte (Teorema A.12 conlui-se que $v>0$ em $B$. Portanto $\left|u_{0}\right|>0$. No seguinte pode-se supor sem perda de generalidade que $u_{0}>0$. 
Teorema 2.18. [6. Theorem 1.3] Suponha $1<p<2^{*}, p \neq 2$ e $\alpha \geq 0$. Então o problema (2.1) admite uma solução ground state. Além disso, qualquer solução ground state u de (2.1) é tal que $u>0$ em $B$ ou $u<0$ em $B$.

Demonstração. A existência da solução segue da Proposição 2.17 juntamente com a Observação 2.15e Teorema 2.16.

\subsection{Resultado de regularidade}

Nesta seção é mostrado que as soluções do problema (2.1) são de classe $C^{2}(\bar{B})$. Para isto, primeiro começamos considerando a equação

$$
-\Delta u=g(., u) \quad \text { em } B
$$

na bola unitária $B \subset \mathbb{R}^{N}$ com uma função Caratheodory $g: B \times \mathbb{R} \rightarrow \mathbb{R}$ (ver Definição A.27). Além disso, supomos que $g$ satisfaz a seguinte condição de crescimento:

$$
|g(x, u)| \leq C\left(1+|u|^{p}\right)
$$

com $p \leq \frac{N+2}{N-2}$, se $N \geq 3$.

Teorema 2.19. Seja $\Omega$ um dominio em $\mathbb{R}^{N}$ e seja $g: \Omega \times \mathbb{R} \rightarrow \mathbb{R}$ uma função de Carathéodory tal que

$$
|g(x, u)| \leq a(x)(1+|u|) \quad \text { q.t.p.em } \Omega,
$$

onde $0<a \in L^{\frac{N}{2}}(\Omega)$. Seja $u \in H_{0}^{1}(\Omega)$ uma solução fraca da equação $-\Delta u=g($., u). Então $u \in L^{r}(\Omega)$ para todo $1 \leq r<\infty$.

Demonstração. Veja [27, pag. 270].

Para aplicar o Teorema 2.19 com dominio a bola unitária $B$, note que se $u \in H_{0}^{1}(B)$ fracamente resolve (2.31) com $g$ uma função de Carathéodory com crescimento polinomial $|g(x, u)| \leq C\left(1+|u|^{p}\right)$ o teorema é satisfeito com

$$
a(x)=\frac{|g(x, u(x))|}{1+|u(x)|} \in L^{\frac{N}{2}}(B) .
$$

Portanto, segue do teorema anterior que $u \in L^{r}(\Omega)$ para todo $1 \leq r<\infty$ e também tendo em vista a condição de crescimento de $g$, deduz-se que $-\Delta u=g(., u) \in L^{r}(B)$ 
com $1<r<\infty$. Pelo Teorema A.21, conclui-se que

$$
u \in W^{2, r}(B) \cap W_{0}^{1, r}(B), \quad \forall 1<r<\infty .
$$

Por outro lado, considerando $r$ suficientemente grande tal que $r>N$ e pelas desigualdades gerais de Sobolev (ver Teorema A.19 quando $k=2$ ). Tem-se

$$
\|u\|_{C^{1, \gamma}} \leq C|u|_{2, r, B}, \quad \text { onde } \gamma=1-\frac{N}{r} \quad \text { e } \quad C=C(B, r, N, \gamma)
$$

Tendo assim,

$$
W^{2, r}(B) \hookrightarrow C^{1, \gamma}(\bar{B}), \quad 0<\gamma<1 .
$$

Então, das estimativas (2.32)-(2.33) resulta que $u \in C^{1, \gamma}(\bar{B})$. Agora se $g$ é uma função Hölder continua, isto é, $g \in C^{0, \beta}(\bar{B})$ com $0<\beta<1$, usando o Corolário A.22, conclui-se que $u \in C^{2, \beta}(\bar{B})$. Portanto $u \in C^{2}(\bar{B})$.

Teorema 2.20. [27, pag. 271] Seja $1<p<2^{*}$ e $u \in H_{0}^{1}(B)$ uma solução fraca do problema (2.1). Então $u \in C^{2}(\bar{B})$.

Demonstração. Define-se a função $g: B \times \mathbb{R} \rightarrow \mathbb{R}$ dada por $g(x, u)=|x|^{\alpha}|u|^{p-2} u$. Assim definido $g$ é uma função de Carathéodory com crescimento polinomial e pelas imersões de sobolev tem-se,

$$
a(x)=\frac{|x|^{\alpha}|u|^{p-1}}{1+|u(x)|} \in L^{\frac{N}{2}}(B)
$$

Logo pelo análise que foi feito acima obtém-se $u \in C^{1, \gamma}(\bar{B})$ e por definição tem-se que $u,|u| \in C^{0, \gamma}(\bar{B})$. Assim, $|u|^{p-2} u \in C^{0, \gamma}(\bar{B})$ e desde que $|x|^{\alpha} \in C^{0, \sigma}(\bar{B})$ com $\sigma=$ $\min \{1, \alpha\}$ resulta $|x|^{\alpha}|u|^{p-2} u \in C^{0, \beta}(\bar{B}) \operatorname{com} \beta=\min \{\gamma, \sigma\}$, de isto pode-se concluir que $g$ é uma função de Hölder continua. Portanto pelo resultado de Regularidade tem-se $u \in C^{2}(\bar{B})$.

Observação: Note que a Proposição 2.17 e os Teoremas 2.18 e 2.20 garantem a existência de solução clássica e positiva para o problema (2.1).

\subsection{Solução radial para equação de Hénon}

O objetivo desta seção é provar a existência de solução radial para o problema (2.1) no caso em que $1<p<2_{\alpha}^{*}$ e $p \neq 2$. O método aqui aplicado é buscar soluções de 
menor energia entre todas as soluções radiais minimizando o funcional $J_{\text {rad }}$ sobre sua variedade de Nehari.

Definição 2.21. Suponha $1<p<2_{\alpha}^{*}$ e $p \neq 2$. Diz-se que u é solução radial fraca de (2.1) se u é ponto critico do funcional $J_{\text {rad }}: H_{0, \mathrm{rad}}^{1}(B) \rightarrow \mathbb{R}$ definido por

$$
J_{\text {rad }}(u)=\frac{1}{2}\|u\|^{2}-\frac{1}{p} \int_{B}|x|^{\alpha}|u|^{p} d x
$$

Este funcional está bem definido e além disso pelo Lema B.8, $J_{\text {rad }} \in C^{1}\left(H_{0, \text { rad }}^{1}(B), \mathbb{R}\right)$ $\mathrm{e}$

$$
J_{\mathrm{rad}}^{\prime}(u) v=\int_{B} \nabla u \cdot \nabla v d x-\int_{B}|x|^{\alpha}|u|^{p-2} u v d x, \quad \forall u, v \in H_{0, \mathrm{rad}}^{1}(B) .
$$

Denota-se por $N_{J_{\mathrm{rad}}}$ a variedade Nehari associada ao funcional $J_{\mathrm{rad}}$, a saber

$$
N_{J_{\mathrm{rad}}}:=\left\{u \in H_{0, \mathrm{rad}}^{1}(B) ; J_{\mathrm{rad}}^{\prime}(u) u=0, u \neq 0\right\}
$$

Define-se o problema de minimização como

$$
c_{J}^{R}:=\inf _{u \in N_{J_{\mathrm{rad}}}} J_{\mathrm{rad}}(u)
$$

desde que $B$ é invariante por rotações, é natural considerar

$$
S_{\alpha, p}^{\mathrm{rad}}:=\inf _{\substack{u \in H_{0, \mathrm{rad}}^{1}(B) \\ u \neq 0}} \frac{\int_{B}|\nabla u|^{2} d x}{\left(\int_{B}|x|^{\alpha}|u|^{p} d x\right)^{2 / p}},
$$

Lema 2.22. Suponha $1<p<2_{\alpha}^{*}$ e $p \neq 2$. Os problemas de minimização (2.34) e (2.35) são equivalentes no seguinte sentido:

(i) Dada uma sequência minimizante $\left(u_{n}\right) \subseteq N_{J_{\mathrm{rad}}}$ para 2.34$),\left(\left|u_{n}\right|_{p, \alpha}^{-1} u_{n}\right)$ é sequência minimizante para (2.35).

(ii) Dada uma sequência minimizante $\left(u_{n}\right)$ para $2.35,\left(\left\|\bar{u}_{n}\right\|^{\frac{2}{p-2}} \bar{u}_{n}\right) \subseteq N_{J_{\mathrm{rad}}}$ é sequência minimizante para (2.34).

(iii) Tem-se a igualdade $c_{J}^{R}=\frac{p-2}{2 p}\left(S_{\alpha, p}^{\mathrm{rad}}\right)^{\frac{p}{p-2}}$.

(iv) A constante $S_{\alpha, p}^{\mathrm{rad}}$ é atingida se e somente se $c_{J}^{R}$ é atingido.

(v) Se $u \in N_{J_{\mathrm{rad}}}$ é tal que $J_{\mathrm{rad}}(u)=c_{J}^{R}$. Então u é solução radial de energia mínima entre todas as soluções radiais para o problema (2.1). Reciprocamente, se u é solução radial de energia mínima de 2.1) então $J_{\mathrm{rad}}(u)=c_{J}^{R}$. 
Demonstração.Segue-se de forma similar como na prova do Lema 2.14 e a Proposição 2.16. Para isto basta observar que $H_{0, \text { rad }}^{1}(B)$ tem imersão compacta em $L^{p}\left(B,|x|^{\alpha}\right)$.

Proposição 2.23. [3, Theorem 3.1] Suponha $1<p<2_{\alpha^{\prime}}^{*} p \neq 2$ e $\alpha>0$. Então a constante de Sobolev $S_{\alpha, p}^{\mathrm{rad}}$ é atingida por uma função em $H_{0, \text { rad }}^{1}(B)$. Além disso, se $S_{\alpha, p}^{\mathrm{rad}}$ é atingido por $u$, então ué $C^{2}(\bar{B}) e|u|>0$ em $B$, isto é, $u>0$ em $B$ ou $u<0$ em $B$.

Demonstração. Pela Proposição 2.9 tem-se que existe $c>0$ tal que $|u|_{p, \alpha} \leq c\|u\|$ para todo $u \in H_{0, \text { rad }}^{1}(B)$, de isto resulta que $S_{\alpha, p}^{\mathrm{rad}}>0$. Seja $\left(u_{n}\right)$ uma sequência minimizante para (2.35), isto é, $S_{\alpha, p}^{\mathrm{rad}}=\lim _{n \rightarrow \infty}\left\|u_{n}\right\|^{2} \operatorname{com}\left|u_{n}\right|_{p, \alpha}=1$. Desde que $H_{0, \text { rad }}^{1}(B)$ é um espaço reflexivo e pelo Teorema de Kakutani (Teorema A.7) existe $u_{0} \in H_{0, \text { rad }}^{1}(B)$ tal que a menos de subsequência $u_{n} \rightarrow u_{0}$ em $H_{0, \text { rad }}^{1}(B)$. A partir de isto e do Teorema de RellichKondrachov (Teorema A.20) tem-se

$$
\left\|u_{0}\right\|^{2} \leq \liminf _{n \rightarrow \infty}\left\|u_{n}\right\|^{2} \quad \text { e } \quad\left|u_{0}\right|_{p, \alpha}=1
$$

Note que pela Proposição 2.9 o limite $u_{0}$ nao pode ser nulo, já que nesse caso temse $\int_{B}|x|^{\alpha}|u|^{p} d x \longrightarrow 0$ o qual é a uma contradição com na segunda parte de (2.36). Portanto

$$
\left\|u_{0}\right\|^{2} \leq \lim _{n \rightarrow \infty}\left\|u_{n}\right\|^{2}=S_{\alpha, p}^{\mathrm{rad}} \leq\left\|u_{0}\right\|^{2} .
$$

A prova da segunda parte da proposição segue de forma análoga como a demostração da Proposição 2.17 .

Teorema 2.24. [23. Theorem 6] Suponha $1<p<2_{\alpha}^{*}$ e $p \neq 2$. Então o problema (2.1) admite uma solução de menor energia entre todas as soluções radias. Além disso, qualquer solução de energia minima radial u de (2.1) tem sinal definido.

Demonstração. A demostração segue da proposição 2.23 juntamente com os items (iv) e (v) do Lema 2.22 que garante a existência de uma solução radial para o problema (2.1) que tem a menor energia entre todas as soluções radiais. Além disso, pelo principio do máximo forte (Teorema A.12 obtém-se $u>0$ ou $u<0$ em $B$.

Até agora tem-se que $u$ é solução fraca para o problema (2.1). No teorema seguinte vai ser mostrado que $u$ é uma solução clássica deste problema.

Teorema 2.25. Seja $1<p<2_{\alpha}^{*} \operatorname{com} p \neq 2$ e $u \in H_{0, \mathrm{rad}}^{1}(B)$ uma solução radial fraca do problema (2.1). Então $u \in C^{2}(\bar{B})$ é uma solução clássica de (2.1). 
Demonstração.Seja $u \in H_{0}^{1}(B)$ é solução do problema (2.1). Define-se a função $g$ : $B \times \mathbb{R} \longrightarrow \mathbb{R}$ dada por $g(x, u)=|x|^{\alpha}|u|^{p-2} u$. Assim, $g$ é uma função de Carathéodory e

$$
|g(x, u)|=|x|^{\alpha}|u|^{p-1-\frac{N+2}{N-2}}|u|^{\frac{N+2}{N-2}}=R(x)|u|^{\frac{N+2}{N-2}},
$$

Aplicando o Teorema 2.7, deduz-se

$$
\begin{aligned}
R(x) & \leq|x|^{\alpha} C \frac{\|u\|^{p-1-\frac{N+2}{N-2}}}{|x|^{\left(p-1-\frac{N+2}{N-2}\right)\left(\frac{N+2}{2}\right)}} \\
& =C_{1}|x|^{\alpha-\left(p-1-\frac{N+2}{N-2}\right)\left(\frac{N+2}{2}\right)}
\end{aligned}
$$

onde $C_{1}=C\|u\|^{p-1-\frac{N+2}{N-2}}$, desde que $2<p<2_{\alpha}^{*}$ tem-se $\alpha-\left(p-1-\frac{N+2}{N-2}\right)\left(\frac{N+2}{2}\right)>0$. Assim de (2.37),

$$
|g(x, u)| \leq C_{1}|u(x)|^{\frac{N+2}{N-2}}=C_{1}|u(x)|^{\frac{4}{N-2}}|u(x)|=a(x)|u(x)|,
$$

onde $a(x)=C_{1}|u(x)|^{\frac{4}{N-2}}$ para todo $x \in B$. Agora vai ser mostrado que $a \in L^{\frac{N}{2}}(B)$. Com efeito,

$$
\begin{aligned}
\int_{B}|a(x)|^{\frac{N}{2}} d x & =\int_{B}\left(C_{1}|u(x)|^{\frac{4}{N-2}}\right)^{\frac{N}{2}} d x=C_{1}^{\frac{N}{2}} \int_{B}|u(x)|^{\frac{2 N}{N-2}} d x \\
& =C_{1}^{\frac{N}{2}}\|u\|_{2^{*}}^{\frac{2 N}{N-2}}<\infty
\end{aligned}
$$

Logo,

$$
|g(x, u)| \leq a(x)(1+|u(x)|), \operatorname{com} a \in L^{\frac{N}{2}}(B) .
$$

Agora usando a [12, Proposition 5.1], segue-se então que $u \in H_{0}^{1}(B)$ e é solução de (2.1) no sentido de $H_{0}^{1}(B)$. A partir deste ponto a prova segue como na prova do Teorema 2.20 .

Observação: Note que os Teoremas 2.24 e 2.25 garantem a existência de solução clássica não trivial com sinal definido para o problema (2.1). Sem perda de geralidade pode-se considerar como soluções positivas.

Observe também que para $N=1,2$, os funcionais $J$ e $J_{\text {rad }}$ acima estão bem definidos para todo $1<p<\infty$, isto pois $H_{0}^{1}(B) \hookrightarrow L^{q}(B)$ para todo $1<q<\infty$. Portanto, existirá solução ground state e solucao radial de menor energia para o problema (2.1) quando $N=1,2,1<p<\infty$ e $p \neq 2$. 


\subsection{Caracterizações das soluções ground state}

Considera-se nesta seção $2<p<2^{*}$ e defina

$$
\begin{aligned}
c_{J} & :=\inf _{u \in N_{J}} J(u), \\
c_{1} & :=\inf _{u \in H_{0}^{1} \backslash\{0\}} \max _{t \geq 0} J(t u), \\
c & :=\inf _{\gamma \in \Gamma \max } J(\gamma(t)),
\end{aligned}
$$

onde $\Gamma:=\left\{\gamma \in C\left([0,1], H_{0}^{1}(B)\right): \gamma(0)=0, J(\gamma(1))<0\right\}$. Observe que $c$ é o nível do Passo da Montanha do funcional $J$.

Teorema 2.26. $c_{J}=c_{1}=c>0$. Em particular, o mínimo de $J$ sobre sua variedade de Nehari é o seu nível Passo da Montanha.

\section{Demonstração.}

- $c_{J}=c_{1}$ :

Seja $u \in H_{0}^{1}(B) \backslash\{0\}$. Pela primeira parte do Lema 2.12, tem-se que existe um único $t(u)>0$ tal que $t(u) u \in N_{J}$. De isto $\inf _{u \in N_{J}} J(u) \leq J(t(u) u)$. Assim,

$$
\inf _{u \in N_{J}} J(u) \leq \inf _{u \in H_{0}^{1} \backslash\{0\}} J(t(u) u) .
$$

Por outro lado, seja $u \in N_{J}$, pelo homeomorfismo que acabou-se de mostrar, existe $v \in B$ tal que $u=t(v) v$. Assim, $\inf _{u \in H_{0}^{1} \backslash\{0\}} J(t(u) u) \leq J(u)$. Logo segue-se,

$$
\inf _{u \in H_{0}^{1} \backslash\{0\}} J(t(u) u) \leq \inf _{u \in N_{J}} J(u)
$$

Portanto tem-se $\inf _{u \in H_{0}^{1} \backslash\{0\}} J(t(u) u)=\inf _{u \in N_{J}} J(u)$. De isto e usando o Lema 2.12 . obtém-se

$$
\inf _{u \in H_{0}^{1} \backslash\{0\}} \max _{t \geq 0} J(t u)=\inf _{u \in N_{J}} J(u) .
$$

- $c \leq c_{1}$ :

Seja $u \in H_{0}^{1}(B) \backslash\{0\}$ e considere o camino $\gamma(s)=$ stu com $t$ suficientemente grande. Tendo em vista o Lema 2.12, onde foi mostrado que $J(t u)<0$ para valores de $t$ suficientemente grandes, tem-se $J(\gamma(1))<0$. Assim, $\gamma \in \Gamma$ e pela definição de $c$, obtém-se

$$
c \leq \max _{s \in[0,1]} J(\gamma(s)) \leq \max _{s \geq 0} J(\gamma(s)) \leq \max _{r \geq 0} J(r u) ; \quad \forall u \in H_{0}^{1}(B) \backslash\{0\} .
$$


Então $\quad c \leq \inf _{u \in H_{0}^{1} \backslash\{0\}} \max _{t \geq 0} J(t u)$.

- $c_{J} \leq c$ : Define-se

$$
\begin{aligned}
\Psi: H_{0}^{1}(B) \backslash\{0\} & \rightarrow \mathbb{R} \\
u & \mapsto \Psi(u)=t\left(\frac{u}{\|u\|}\right)-\|u\| .
\end{aligned}
$$

Note que $\Psi$ é continua desde que as funções $\|$.$\| e u \mapsto t(u)$ são contínuas (ver Lema 2.12. É imediato provar que a variedade Nehari associada ao funcional $J$ pode ser vista como o conjunto,

$$
N_{J}=\left\{u \in H_{0}^{1}(B) \backslash\{0\} t\left(\frac{u}{\|u\|}\right)=\|u\|\right\}=\Psi^{-1}(0)
$$

o qual é fechado em $H_{0}^{1}(B) \backslash\{0\}$ e por ende é também fechado em $H_{0}^{1}(B)$. Observe que este conjunto separa $H_{0}^{1}(B) \backslash\{0\}$ em duas componentes, as quais são

$$
\begin{aligned}
U_{1}=\Psi^{-1}((-\infty, 0)) & =\left\{u \in H_{0}^{1}(B) \backslash\{0\}:\|u\|>t\left(\frac{u}{\|u\|}\right)\right\} \\
& =\left\{u \in H_{0}^{1}(B) \backslash\{0\}: J^{\prime}(u) u<0\right\}, \\
U_{2}=\Psi^{-1}((0, \infty)) & =\left\{u \in H_{0}^{1}(B) \backslash\{0\}:\|u\|<t\left(\frac{u}{\|u\|}\right)\right\} \\
& =\left\{u \in H_{0}^{1}(B) \backslash\{0\}: J^{\prime}(u) u>0\right\} .
\end{aligned}
$$

Note que $U_{1}$ e $U_{2}$ são conjuntos abertos em $H_{0}^{1}(B) \backslash\{0\}$ e além disso o conjunto $U_{2}^{*}=U_{2} \cup\{0\}$ é aberto em $H_{0}^{1}(B)$.

Afirmação: Se $u \in U_{2}^{*}$, então $J(u) \geq 0$.

De fato,

- Se $u=0$, entao tem-se $J(u) \geq 0$.

- Se $u \in U_{2}$ então $J^{\prime}(u) u>0$. Se suponha-se que $t u \in U_{1}$ para algum $t$ tal que $0<t<t(u)$, então tem-se $J^{\prime}(t u) t u<0$. Assim $g^{\prime}(t)<0$ o qual a partir do Lema 2.12. tem-se uma contradição. Portanto $t u \in U_{2}$ e além disso $g^{\prime}(t)>0$. Logo de isto resulta que $g$ é crescente em $[0, t(u)]$ e como $g(0)=0$, então $g(t) \geq 0$ para todo $t \in[0, t(u)]$. Assim, $J(t u) \geq 0$ para todo $t \in[0, t(u)]$, em particular para $t=1$. Portanto $J(u) \geq 0$.

Assim a afirmação está provada. 
Agora seja $\gamma \in \Gamma$, pela definição tem-se $\gamma(0)=0$ e $J(\gamma(1))<1$. Como $\gamma(1) \in H_{0}^{1}(B)$, então pela afirmação conclui-se que $\gamma(1) \notin U_{2}^{*}$. Assim $\gamma(1) \in U_{1}$ o qual implica que o camino $\gamma$ tem que passar por $N_{J}$, deste modo existe $t_{0} \in[0,1]$ tal que $\gamma\left(t_{0}\right) \in N_{J}$ e

$$
\inf _{u \in N_{J}} J(u) \leq J\left(\gamma\left(t_{0}\right)\right) \leq \max _{t \in[0,1]} J(\gamma(t))
$$

Portanto

$$
c_{J} \leq \inf _{\gamma \in \Gamma} \max _{t \in[0,1]} J(\gamma(t)) .
$$




\section{Capítulo}

\section{3}

\section{O caso $1<p<2$}

O capítulo anterior garante a existência de solução positiva para o problema (3.1) no caso $1<p<2_{\alpha}^{*} \operatorname{com} p \neq 2$. Neste capítulo é mostrado a unicidade de solução positiva no caso $1<p<2$. Para isso usamos algumas das idéias contidas em [22, Section 4]. Como consequência desse resultado de unidade e da invariância do Laplaciano por transformações ortogonais, mostramos que a solução positiva de (3.1) no caso em que $1<p<2$ é radial.

Consideremos a equação de Hénon,

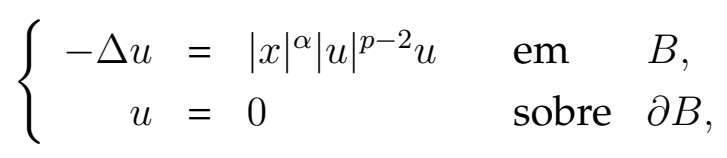

onde $B$ denota a bola unitária aberta de $\mathbb{R}^{N}, N \geq 1, \alpha>0$ e $1<p<2$.

Teorema 3.1. Seja $1<p<2$. Então o problema (3.1) possui uma única solução clássica positiva.

Demonstração. A existência de uma solução positiva para o problema (3.1) foi feita no Capítulo 2, como consequência dos Teoremas 2.18 e 2.20. Suponha que $u$ e $v$ sejam soluções clássicas positivas de (3.1). Para mostrar a unicidade basta mostrar que $u \geq v$.

Seja

$$
S=\{0<s<1 ; u>s v \text { em } B\} \quad \text { e } \quad s_{*}=\sup S .
$$

Note que pelo pelo Lema de Hopf (ver Lema A.10 tem-se que $\frac{\partial u}{\partial \eta}<0, \frac{\partial v}{\partial \eta}<0$ sobre $\partial B$. Logo, o Lema A.11 fornece a existência de $\varepsilon>0$ tal que $u>\varepsilon v$ em $B$. Assim o conjunto $S$ é não vazio.

Afirmação: O conjunto $S$ é um intervalo aberto. Em particular, $s_{*} \notin S$. 
Claramente $S$ é um intervalo, pois se $s \in S$ e $0<s^{\prime}<s$, então $s^{\prime} \in S$. Para provar que $S$ é aberto, tome $s \in S$ e observe que

$$
\begin{aligned}
-\Delta(u-s v) & =|x|^{\alpha} u^{p-1}-|x|^{\alpha} s v^{p-1}=|x|^{\alpha} u^{p-1}-|x|^{\alpha} s^{2-p}(s v)^{p-1} \\
& \geq|x|^{\alpha} s^{2-p} u^{p-1}-|x|^{\alpha} s^{2-p}(s v)^{p-1}=|x|^{\alpha} s^{2-p}\left[u^{p-1}-(s v)^{p-1}\right] \geq 0 \quad \text { em } B,
\end{aligned}
$$

e $u-s v=0$ sobre $\partial B$. Além disso, as desigualdades acima são estritas se $x \in B \backslash\{0\}$. Então o Lema de Hopf garante que $\frac{\partial(u-s v)}{\partial \eta}<0$ sobre $\partial B$. Assim, $u-s v>0$ e $v>0$ em $B$ e $\frac{\partial(u-s v)}{\partial \eta}<0$ e $\frac{\partial v}{\partial \eta}<0$ sobre $\partial B$. Agora fazendo uso do Lema A.11 para $u-s v$ e $v$ obtém-se que existe $\varepsilon>0$ tal que $u-s v>\varepsilon v$ em $B$. Assim $u-(s+\varepsilon) v>0$ em $B$, isto é, $s+\varepsilon \in S$. Portanto $S$ é um intervalo aberto.

Para provar o resultado de unicidade é suficiente mostrar que $s_{*}=1$. Pois se $s_{*}=1$, então $S=(0,1)$. Assim para qualquer $x \in B$ pode-se considerar $\left(s_{n}\right)$ uma sequência em $S=(0,1)$ tal que $s_{n} \longrightarrow 1$ quando $n \longrightarrow \infty$. Como $u(x)>s_{n} v(x)$ para todo $n \in \mathbb{N}$, obtém-se que $u(x) \geq v(x)$. Assim, $s_{*}=1$ garante $u \geq v$.

Procedendo por contradição, suponha que $s_{*}<1$. A suposição $s_{*}<1$ permite mostrar que $s_{*} \in S$, o que contradiz a afirmação anterior.

Se $s_{*}<1$, então:

$$
\begin{aligned}
-\Delta\left(u-s_{*} v\right) & =|x|^{\alpha} u^{p-1}-|x|^{\alpha} s_{*} v^{p-1}=|x|^{\alpha}\left[u^{p-1}-s_{*}^{2-p}\left(s_{*} v\right)^{p-1}\right] \\
& \geq|x|^{\alpha}\left[s_{*}^{2-p} u^{p-1}-s_{*}^{2-p}\left(s_{*} v\right)^{p-1}\right]=|x|^{\alpha} s_{*}^{2-p}\left[u^{p-1}-\left(s_{*} v\right)^{p-1}\right] \geq 0 \quad \text { em } B,
\end{aligned}
$$

onde a primeira desigualdade é estrita em $B \backslash\{0\}$. Além disso, $u-s_{*} v=0$ em $\partial B$. Então pelo princípio do máximo forte tem-se $u>s_{*} v$ em $B$, isto é, $s_{*} \in S$.

Observação: Seja $T: \mathbb{R}^{N} \rightarrow \mathbb{R}^{N}$ uma transformação linear e $T=\left(T_{i j}\right), i, j=1, \ldots, N$, sua representação matricial de $T$ em relação à base canônica de $\mathbb{R}^{N}$. Lembramos que $T \in O(N)$ se, e somente se, as linhas (colunas) de $T$ formam uma base ortonormal de $\mathbb{R}^{N}$.

Lema 3.2. Sejam $\Omega \subset \mathbb{R}^{N}$ um aberto radialmente simétrico, $u \in C^{2}(\Omega)$ e $g \in O(N)$. Então

$$
\Delta(u \circ g)(x)=\Delta u(g x), \quad \forall x \in \Omega
$$

Demonstração. Seja $v=u \circ g$ e $g=\left(g_{i j}\right), i, j=1, \ldots, N$ a representação matricial de $g$ em relação à base canônica de $\mathbb{R}^{N}$. Então pela regra da cadeia tem-se $\nabla v(x)=\nabla u(g x) g$, 
isto é,

$$
v_{k}(x)=\sum_{i=1}^{N} u_{i}(g x) g_{i k}, \quad \forall k=1,2, \ldots, N .
$$

Novamente pela regra da cadeia,

$$
\nabla v_{k}(x)=\sum_{i=1}^{N} \nabla u_{i}(g x) g g_{i k}
$$

isto é,

$$
v_{k j}(x)=\sum_{i=1}^{N} \sum_{l=1}^{N} u_{i l}(g x) g_{l j} g_{i k}
$$

Logo, para $j=k$ tem-se

$$
v_{k k}(x)=\sum_{i, l=1}^{N} u_{i l}(g x) g_{l k} g_{i k}=\sum_{i=1}^{N} u_{i i}(g x) g_{i k}^{2}+\sum_{i \neq l ; i, l=1}^{N} u_{i l}(g x) g_{l k} g_{i k}
$$

Agora somando em $k$ e tendo em vista a observação acima, obtém-se

$$
\Delta(u \circ g)(x)=\sum_{k=1}^{N} v_{k k}(x)=\sum_{i=1}^{N}\left[u_{i i}(g x) \sum_{k=1}^{N} g_{i k}^{2}\right]+\sum_{\substack{i \neq l \\ i, l=1}}^{N}\left[u_{i l}(g x) \sum_{k=1}^{N} g_{l k} g_{i k}\right]=\Delta u(g x) .
$$

Corolário 3.3. Se $1<p<2$ então a solução clássica positiva de (3.1) é radialmente simétrica.

Demonstração. Seja $u$ a única solução positiva de (3.1) e $g \in O(N)$. Defina $v=u \circ g$. Então pelo Lemma 3.2

$$
\left\{\begin{array}{l}
-\Delta v(x)=-\Delta u(g x)=|g x|^{\alpha} u^{p-1}(g x)=|x|^{\alpha} v^{p-1}(x), \quad \forall x \in B, \\
v>0 \text { em } B \quad \text { e } \quad v=0 \text { sobre } \partial B
\end{array}\right.
$$

isto é, $v$ também é uma solução positiva de (3.1). Então, pela unicidade de solução positiva para (3.1), segue-se que $u=v=u \circ g$ para toda $g \in O(N)$, isto é, $u$ é radialmente simétrica. 
O caso $1<p<2$ 


\section{Capítulo 4 \\ Quebra de simetria dos ground states}

Este capítulo apresenta resultados sobre a equação de Hénon para o caso subcrítico e superlinear, isto é, $2<p<2^{*}$. Note que para este intervalo o Capítulo 2 garante a existência de solução ground state e que qualquer solução ground state tem sinal definido, isto é, é positiva em $B$ ou negativa em $B$. O objetivo deste capítulo é estudar questão da simetria desta classe especial de soluções. Na Seção 4.1 é mostrado que no caso em que $\alpha$ é suficientemente grande, qualquer solução ground state não é radialmente simétrica, informação esta que constrata com a radialidade do problema (2.1). Observe que este fenômeno de quebra de simetria dá origem a, pelo menos, duas soluções positivas para (2.1) quando $2<p<2^{*}$ e $\alpha$ for suficientemente grande. Por último, na Seção 4.2, é mostrado que qualquer solução ground state exibe a simetria de Schwarz folheada.

\subsection{Solução não-radial para equação de Hénon}

Nesta seção provaremos que a solução ground state, cuja existência foi provada no capítulo anterior, não é radialmente simétrica, isto sempre que $2<p<2^{*}$ e $\alpha$ for suficientemente grande.

Lema 4.1. [3, Lemma 3.2] Seja $\beta \in(0,1]$ e para $u: B \rightarrow \mathbb{R}$, define-se $\left(T_{\beta} u\right)(x)=u\left(|x|^{\beta-1} x\right)$. Então $T_{\beta}\left(H_{0, \mathrm{rad}}^{1}\right) \subseteq H_{0, \mathrm{rad}}^{1}$ e para $p \in\left[2,2_{\alpha}^{*}\right]$ tem-se

$$
S_{\alpha, p}^{r a d}=\inf _{w \in T_{\beta}\left(H_{0, r a d}^{1} \backslash\{0\}\right)} \frac{1}{\beta^{1+2 / p}} \frac{\int_{B}|\nabla w|^{2}|x|^{(\beta-1)(N-2)} d x}{\left(\int_{B}|w|^{p}|x|^{\beta(\alpha+N)-N} d x\right)^{2 / p}} .
$$


Demonstração. Seja $w \in T_{\beta}\left(H_{0, \text { rad }}^{1} \backslash\{0\}\right)$. Então existe $u \in H_{0, \text { rad }}^{1}$ tal que $w=T_{\beta} u$. Note que $u(x)=v(r) \operatorname{com} r=|x|$ e $v \in W^{1,2}\left((0,1), t^{N-1}\right)$. Também

$$
w(x)=\left(T_{\beta} u\right)(x)=u\left(|x|^{\beta-1} x\right)=v\left(|x|^{\beta}\right) .
$$

Assim $w \in H_{0, \text { rad }}^{1}(B)$. Desde que $u$ é função radial note também que

$$
\int_{B}|x|^{\alpha}|u(x)|^{p} d x=\omega_{N} \int_{0}^{1}|v(r)|^{p} r^{\alpha+N-1} d r
$$

onde $\omega_{N}$ é a área de superfície da esfera unitária $S^{N-1} \subset \mathbb{R}^{N}$. Fazendo mudança de variável $r=t^{\beta}$, tem-se

$$
\begin{aligned}
\omega_{N} \int_{0}^{1}|v(r)|^{p} r^{\alpha+N-1} d r & =\omega_{N} \int_{0}^{1}\left|v\left(t^{\beta}\right)\right|^{p} t^{\beta(\alpha+N-1)} \beta t^{\beta-1} d t=\beta \omega_{N} \int_{0}^{1}\left|v\left(t^{\beta}\right)\right|^{p} t^{\beta(\alpha+N)-1} d t \\
& =\beta \omega_{N} \int_{0}^{1}\left|v\left(t^{\beta}\right)\right|^{p} t^{\beta(\alpha+N)-N} t^{N-1} d t=\beta \int_{B}|w(x)|^{p}|x|^{\beta(\alpha+N)-N} d x .
\end{aligned}
$$

Logo das duas últimas igualdades segue-se

$$
\int_{B}|x|^{\alpha}|u|^{p} d x=\beta \int_{B}|w(x)|^{p}|x|^{\beta(\alpha+N)-N} d x .
$$

Observe-se que $|\nabla u|,|\nabla w| \in H_{0, \text { rad }}^{1}(B)$ e

$$
\begin{gathered}
\int_{B}|\nabla u(x)|^{2} d x=\omega_{N} \int_{0}^{1}\left|v^{\prime}(r)\right|^{2} r^{N-1} d r, \\
\int_{B}|\nabla w(x)|^{2}=\omega_{N} \int_{0}^{1}\left|v^{\prime}\left(r^{\beta}\right) \beta r^{(\beta-1)}\right|^{2} r^{N-1} d r .
\end{gathered}
$$

Fazendo mudança de variável $r=t^{\beta}$ em (4.2) e usando (4.3), obtém-se

$$
\begin{aligned}
\int_{B}|\nabla u(x)|^{2} d x & =\omega_{N} \int_{0}^{1}\left|v^{\prime}\left(t^{\beta}\right)\right|^{2}\left(t^{\beta}\right)^{N-1} \beta t^{\beta-1} d t \\
& =\beta^{-1} \omega_{N} \int_{0}^{1}\left|v^{\prime}\left(t^{\beta}\right)\right|^{2} \beta^{2} t^{2(\beta-1)} t^{\beta(N-1)+\beta-1-2(\beta-1)} d t \\
& =\beta^{-1} \omega_{N} \int_{0}^{1}\left|v^{\prime}\left(t^{\beta}\right)\right|^{2} \beta^{2} t^{2(\beta-1)} t^{(2-N)(1-\beta)} t^{N-1} d t \\
& =\beta^{-1} \int_{B}|\nabla w(x)|^{2}|x|^{(2-N)(1-\beta)} d x .
\end{aligned}
$$


Das expressões (4.1) e (4.4) resulta

$$
\frac{\int_{B}|\nabla u|^{2} d x}{\left(\int_{B}|x|^{\alpha}|u|^{p} d x\right)^{2 / p}}=\frac{\int_{B}|\nabla w|^{2}|x|^{(2-N)(1-\beta)} d x}{\beta^{1+2 / p}\left(\int_{B}|w|^{p}|x|^{\beta(\alpha+N)-N} d x\right)^{2 / p}}
$$

Assim

$$
S_{\alpha, p}^{r a d}=\inf _{\substack{\left.u \in H_{0, \mathrm{rad}}^{1}(B)\right) \\ u \neq 0}} \frac{\int_{B}|\nabla u|^{2} d x}{\left(\int_{B}|x|^{\alpha}|u|^{p} d x\right)^{2 / p}}=\inf _{\substack{w \in T_{\beta}\left(H_{0, \mathrm{rad}}^{1}(B)\right) \\ w \neq 0}} \frac{\int_{B}|\nabla w|^{2}|x|^{(2-N)(1-\beta)} d x}{\beta^{1+2 / p}\left(\int_{B}|w|^{p}|x|^{\beta(\alpha+N)-N} d x\right)^{2 / p}} .
$$

Proposição 4.2. [25, Theorem 4.1] Suponha $2<p<2_{\alpha}^{*}$. Se $N \geq 2$, existe $\tilde{C}$ dependendo de $N$ e p tal que $S_{\alpha, p}^{r a d} \sim \tilde{C}\left(\frac{\alpha+N}{N}\right)^{1+\frac{2}{p}}$ quando $\alpha \longrightarrow \infty$.

Demonstração. Sejam $u$ e $w=T_{\beta} u$. No Lema 4.1 escolha $\beta=\frac{N}{N+\alpha}$ e tem-se

$$
\beta^{1+2 / p} S_{\alpha, p}^{r a d}=\inf _{w \in T_{\beta}\left(H_{0, \text { rad }}^{1} \backslash\{0\}\right)} \frac{\int_{B}|\nabla w|^{2}|x|^{(\beta-1)(N-2)} d x}{\left(\int_{B}|w|^{p}|x|^{\beta(\alpha+N)-N} d x\right)^{2 / p}} .
$$

Por outro lado, para todo $0 \leq \beta \leq 1$ definina

$$
c_{\beta}:=\inf _{\left.w \in T_{\beta}\left(H_{0, \mathrm{rad}}^{1} \backslash\{0\}\right)\right)} \frac{\int_{B}|\nabla w|^{2}|x|^{(2-N)(1-\beta)} d x}{\left(\int_{B}|w|^{2} d x\right)^{2 / p}} .
$$

$c_{\beta}$ está bem definida, pois ao menos existe um $w \in H_{0}^{1}(B)$ definida na forma que seja constante em $B_{\varepsilon}(0)$ onde $0<\varepsilon<1$. Observe que $c_{\beta}$ é monótona não-crescente sobre $[0,1]$. Assim, existe $\tilde{C}>0$ tal que $\tilde{C}=\lim _{\beta \rightarrow 0^{+}} c_{\beta}$. Tendo em vista as duas últimas igualdades obtém-se

$$
\beta^{1+2 / p} S_{\alpha, p}^{r a d}=c_{\beta}
$$

Assim,

$$
\lim _{\alpha \rightarrow \infty}\left(\frac{N}{N+\alpha}\right)^{1+2 / p} S_{\alpha, p}^{r a d}=\lim _{\beta \rightarrow 0^{+}} \beta^{1+2 / p} S_{\alpha, p}^{r a d}=\lim _{\beta \rightarrow 0^{+}} c_{\beta}=\tilde{C} .
$$

Ou equivalentemente

$$
\tilde{C}\left(\frac{\alpha+N}{N}\right)^{1+2 / p} \sim S_{\alpha, p}^{r a d}, \quad \text { quando } \alpha \longrightarrow \infty
$$


Teorema 4.3. [25. Theorem 4.2] Se $N \geq 2$, para todo $2<p<2^{*}$ existe $\alpha^{*} \geq 0$ tal que $S_{\alpha, p}<S_{\alpha, p}^{r a d}$, para $\alpha>\alpha^{*}$.

Demonstração. Seja $u \in C_{c}^{\infty}(B), u \geq 0, u \neq 0$, e defina $u_{\alpha}(x):=u\left(\alpha\left(x-x_{\alpha}\right)\right)$ onde $x_{\alpha}=\left(1-\frac{1}{\alpha}, 0,0 \ldots, 0\right)$. Tem-se

$$
\int_{B}\left|\nabla u_{\alpha}\right|^{2} d x=\int_{B}\left|\nabla u\left(\alpha\left(x-x_{\alpha}\right)\right)\right|^{2} d x
$$

Fazendo mudança de variável $y=\alpha\left(x-x_{\alpha}\right)$

$$
\int_{B}\left|\nabla u_{\alpha}\right|^{2} d x=\frac{\alpha^{2}}{\alpha^{N}} \int_{B}|\nabla u(y)|^{2} d y=\alpha^{2-N} \int_{B}|\nabla u(x)|^{2} d x
$$

e também desde que $\alpha\left(x-x_{\alpha}\right) \in B$ então tem-se $|x| \geq 1-\frac{2}{\alpha}$ e novamente fazendo mudança de variável $y=\alpha\left(x-x_{\alpha}\right)$, obtém-se

$$
\begin{aligned}
\int_{B}|x|^{\alpha} u_{\alpha}^{p} d x & \geq \int_{B}\left(1-\frac{2}{\alpha}\right)^{\alpha} u_{\alpha}^{p}(x) d x=\left(1-\frac{2}{\alpha}\right)^{\alpha} \int_{B} u^{p}\left(\alpha\left(x-x_{\alpha}\right)\right) d x \\
& =\left(1-\frac{2}{\alpha}\right)^{\alpha} \int_{B} u^{p}(y) \frac{1}{\alpha^{N}} d y=\left(1-\frac{2}{\alpha}\right)^{\alpha} \alpha^{-N} \int_{B} u^{p}(x) d x
\end{aligned}
$$

Das duas últimas igualdades resulta

$$
S_{\alpha, p} \leq \frac{\int_{B}\left|\nabla u_{\alpha}\right|^{2} d x}{\left(\int_{B}|x|^{\alpha}\left|u_{\alpha}\right|^{p} d x\right)^{2 / p}} \leq \frac{\alpha^{2-N} \int_{B}|\nabla u|^{2} d x}{\left[\left(1-\frac{2}{\alpha}\right)^{\alpha} \alpha^{-N} \int_{B} u^{p} d x\right]^{2 / p}} \leq C \alpha^{2-N-\frac{2 N}{p}}, \quad \forall \alpha>\alpha^{*},
$$

onde $C=\frac{\int_{B}\left|\nabla u_{\alpha}\right|^{2} d x}{\left[\left(1-\frac{2}{\alpha}\right)^{\alpha} \int_{B} u^{p} d x\right]^{2 / p}}$. Por outro lado, em 4 para $\frac{\tilde{C}}{2}>0$, existe $\alpha^{*}=$ $\alpha^{*}(N, p) \geq 0$ tal que

$$
\left(\frac{\alpha+N}{N}\right)^{1+2 / p} \tilde{C}<S_{\alpha, p}^{r a d}
$$

Assim

$$
c \alpha^{1+\frac{2}{p}}<S_{\alpha, p}^{r a d}, \quad \forall \alpha>\alpha^{*}
$$

onde $c=\tilde{C} \frac{1}{N^{1+2 / p}}$. Observe que $1+\frac{2}{p}>2-N+\frac{2 N}{p}$. De isto e de fato que $c, C>0$ tem-se que $c \alpha^{1+2 / p}>C \alpha^{2-N-\frac{2 N}{p}}$ quando $\alpha>\alpha^{*}$. Assim, das estimativas 4.7) e 4.8) fica demostrado o teorema.

Teorema 4.4. Se $N \geq 2$ e $2<p<2^{*}$, então existe $\alpha^{*} \geq 0$ tal que qualquer solução ground state da equação de Hénon é não-radial quando $\alpha>\alpha^{*}$. 
Demonstração. Dos Teoremas 2.18 e 2.20, tem-se garantida a existência de uma função $u \in H_{0}^{1}(B)$, solução clássica de energia mínima para $2<p<2^{*}$ e pelo item (iii) do Lema 2.14. $J(u)=c_{J}=\frac{p-2}{2 p} S_{\alpha, p}^{\frac{p}{p-2}}$. Também os Teoremas 2.24 e 2.25 garantem a existência de uma função $v \in H_{0 \text {,rad }}^{1}$ solução clássica de energia mínima entre todas as soluções radiais, a qual satisfaz $J(v)=c_{J}^{r a d}=\frac{p-2}{2 p}\left(S_{\alpha, p}^{\mathrm{rad}}\right)^{\frac{p}{p-2}}$.

Por outro lado, note que pelo Teorema 4.3, existe $\alpha^{*} \geq 0$ tal que para $\alpha>\alpha^{*}$ resulta

$$
J(u)<J(v)
$$

Se $u$ fosse uma solução radial, então teríamos uma contradição com a minimalidade de $J(v)$.

\subsection{Simetria de Schwarz folheada}

A prova aqui apresentada segue idéias contidas nos artigos [5, 26, 4], algumas das quais foram simplificadas em [7].

Será denotado por $\mathcal{H}$ a família de todos os semi-espaços afins fechados em $\mathbb{R}^{N} \mathrm{e}$ por $\mathcal{H}_{*}$ o subconjunto dos elementos $H \in \mathcal{H}$ tais que $0 \in \partial H$. Para cada $H \in \mathcal{H}$, $\sigma_{H}: \mathbb{R}^{N} \rightarrow \mathbb{R}^{N}$ denotará a reflexão com respeito ao hiperplano $\partial H$.

Definição 4.5. A polarização de uma função mensurável não-negativa $u: \mathbb{R}^{N} \rightarrow \mathbb{R}$ com respeito a $H$ é definida por

$$
u_{H}(x)= \begin{cases}\max \left\{u(x), u\left(\sigma_{H}(x)\right)\right\}, & x \in H, \\ \min \left\{u(x), u\left(\sigma_{H}(x)\right)\right\}, & x \in \mathbb{R}^{N} \backslash H .\end{cases}
$$

Seja $P \in S^{N-1}=\left\{x \in \mathbb{R}^{N}:|x|=1\right\}$. Defina

$$
\mathcal{H}_{P}:=\left\{H \in \mathcal{H}_{0}: P \in \operatorname{int}(H)\right\}
$$

Além disso, para $r>0$ e denotamos por $\mu_{r}$ a medida standard sobre $\partial B_{r}(0)$. A simetrização $A^{P}$ de um conjunto $A \subseteq \partial B_{r}(0)$ com respeito a $P$ é definido como a bola geodésica fechada em $\partial B_{r}(0)$ centrada em $r P$, que satisfaz $\mu_{r}\left(A^{P}\right)=\mu_{r}(A)$. Para uma função contínua $u: \mathbb{R}^{N} \rightarrow \mathbb{R}$ a simetrização de Schwarz folheada $u_{P}: \mathbb{R}^{N} \rightarrow \mathbb{R}$ de $u$ com respeito a $P$ é definida pela condição,

$$
\left\{u_{P} \geq t\right\} \cap \partial B_{r}(0)=\left[\{u \geq t\} \cap \partial B_{r}(0)\right]^{P}, \quad \forall r>0, t \in \mathbb{R} .
$$


Para mais detalhes sobre a boa definição de $u_{P}$ veja [4, pag. 4].

Se $u=u_{P}$, então diz-se que $u$ tem a simetria de Schwarz folheada com respeito a $P$. Observação: No caso em que $u: \mathbb{R}^{N} \rightarrow \mathbb{R}$ é contínua observamos que $u$ tem a simetria de Schwarz folheada com respeito algum ponto $P \in S^{N-1}$ se, e somente se:

- $u$ é axialmente simétrica com respeito ao eixo $\mathbb{R} P=\{r P ; r \in \mathbb{R}\}$.

- Para todo $r>0$, u é não crescente no ângulo polar $\theta=\arccos \frac{x}{|x|} \cdot P$, para $x \in$ $S_{r}^{N-1}=\left\{y \in \mathbb{R}^{N}:|y|=r\right\}$.

Proposição 4.6. Seja $u \in C\left(\mathbb{R}^{N}\right)$ e $P \in S^{N-1}$. Então, $u=u_{P}$ se, somente se, $u=u_{H}$ para todo $H \in \mathcal{H}_{P}$.

Demonstração. Apenas faremos a prova para o caso $N=2$. Para o caso geral $N \geq 2$ a prova é feita usando composição de reflexões para reduzir a prova ao caso $N=2$.

Seja $H \in \mathcal{H}_{P}$, suponha $x \in \mathbb{R}^{N}$ e dotamos $x^{\prime}=\sigma_{H}(x)$. Seja $\theta$ o ângulo formado por $P$ e $x$, e $\theta^{\prime}$ o ângulo formado por $P$ e $x^{\prime}$. Na Fig. 1 note que $\theta^{\prime} \geq \theta$. Então como $u$ possui simetria de Schwarz folheada com respeito a $P$, pela segunda condição da observação acima tem-se que $u\left(\sigma_{H}(x)\right) \leq u(x)$. Portanto, $u_{H}=u$.

Agora será provada a recíproca. Primeiramente vai ser provado que $u$ é axialmente simétrica em relação a $\mathbb{R} P$. De fato, seja $x \in \mathbb{R}^{N} \backslash\{0\}$ e $x^{\prime}$ a reflexão de $x$ em relação à $\mathbb{R} P$, veja Fig. 2. Sejam também $H_{n}, \hat{H}_{n} \in \mathcal{H}_{P}$ como na Fig. 2 e $x_{n}^{\prime}=\sigma_{H_{n}}(x)$ e $\hat{x}_{n}=\sigma_{\hat{H}_{n}}(x)$. Como $u=u_{H}$ para todo $H \in \mathcal{H}_{P}$ tem-se que

$$
u\left(x_{n}^{\prime}\right) \leq u(x) \leq u\left(\hat{x}_{n}\right), \quad \forall n \in \mathbb{N}
$$

uma vez que $H_{n}, \hat{H}_{n} \in \mathcal{H}_{P}$. Agora fazendo $H_{n}$ e $\hat{H}_{n}$ tenderem para $\mathbb{R} P$ quando $n \rightarrow \infty$ temos que $x_{n}^{\prime} \rightarrow x^{\prime}$ e $\hat{x}_{n} \rightarrow x^{\prime}$ quando $n \rightarrow \infty$. Então, por 4.9) e da continuidade de $u$ segue-se que $u(x)=u\left(x^{\prime}\right)$.

Resta provar a segunda condição da observação acima. Para isto, sejam $x, y \in$ $B \backslash\{0\}, \operatorname{com}|x|=|y|=r$ e $\theta, \theta^{\prime}$ os ângulos formados por $\mathbb{R} P \operatorname{com} x$ e $\mathbb{R} P \operatorname{com} y$ respetivamente, como mostra a Fig. 3. Considere $H_{x y}$ tal que $x, P \in H_{x y}$ e $\sigma_{H_{x y}}(x)=y$. Pela hipótese tem-se $u=u_{H_{x y}}$ e portanto

$$
u(x)=u_{H_{x y}}(x)=\max \left\{u(x), u\left(\sigma_{H_{x y}}\right)(x)\right\}=\max \{u(x), u(y)\} \geq u(y) .
$$

Portanto, $u(x) \geq u(y)$. Assim está provada a proposição. 

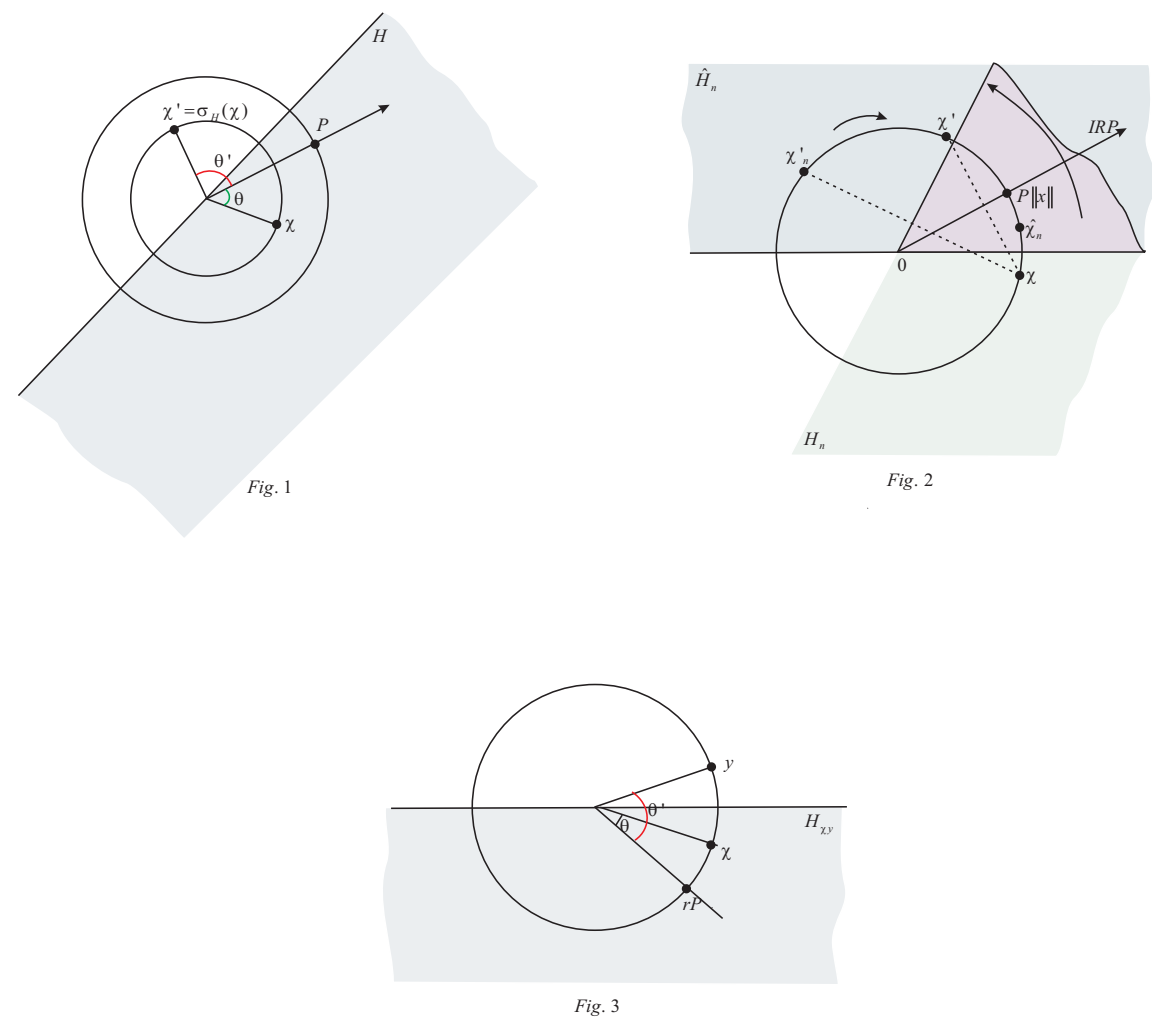

Proposição 4.7. Seja $H \in \mathcal{H}_{*}$. Se $u \in H_{0}^{1}(B)$, então $u_{H} \in H_{0}^{1}(B)$ e $\left|\nabla u_{H}\right|_{2}=|\nabla u|_{2}$.

Demonstração. Seja $v=u \circ \sigma_{H}$, tem-se $u-v \in H_{0}^{1}(H \cap B)$ e também $|u-v| \in$ $H_{0}^{1}(H \cap B)$. De forma similar tem-se $|u-v| \in H_{0}^{1}\left(\sigma_{H}(H) \cap B\right)$. Seja $f:=\mathcal{X}_{H \cap B}|u-v|$ e $g:=\left(\mathcal{X}_{H \cap B}-1\right)|u-v|$ assim definidos $f, g \in H_{0}^{1}(B)$. Note que $u_{H}=\frac{1}{2}(u+v+f+g)$. Então obtém-se $u_{H} \in H_{0}^{1}(B)$. Agora observe que,

$$
\nabla u_{H}(x)\left\{\begin{array}{rll}
\nabla u(x) & \text { sobre } & (H \cap\{u \geq v\}) \cup\left(\sigma_{H}(H) \cap\{u<v\}\right) \\
\nabla u\left(\sigma_{H}(x)\right) & \text { sobre } & (H \cap\{u<v\}) \cup\left(\sigma_{H}(H) \cap\{u \geq v\}\right),
\end{array}\right.
$$

$\mathrm{e}$

$$
\begin{aligned}
& \{x \in H / u(x)<v(x)\}=\sigma_{H}\left(\left\{x \in \sigma_{H}(H) / u(x)>v(x)\right\}\right) \\
& \left\{x \in \sigma_{H}(H) / u(x) \geq v(x)\right\}=\sigma_{H}(\{x \in H / u(x) \leq v(x)\}) .
\end{aligned}
$$

Em seguida usando as duas últimas igualdades e fazendo mudança de variável $y=\sigma_{H}(x)$ implica, 


$$
\begin{aligned}
\int_{H \cap\{u<v\}}\left|\nabla u\left(\sigma_{H}(x)\right)\right|^{2} d x & =\int_{\left.\sigma_{H}\left(\left\{x \in \sigma_{H}(H) / u>v\right)\right\}\right)}\left|\nabla u\left(\sigma_{H}(x)\right)\right|^{2} .1 d x \\
& =\int_{\left.\left\{y \in \sigma_{H}(H) / u>v\right)\right\}}|\nabla u(y)|^{2} d y,
\end{aligned}
$$

de forma similar obtém-se

$$
\int_{\sigma_{H}(H) \cap\{u \geq v\}}\left|\nabla u\left(\sigma_{H}(x)\right)\right|^{2} d x=\int_{\left.\left\{y \in \sigma_{H}(H) / u \leq v\right)\right\}}|\nabla u(y)|^{2} d y,
$$

Logo, fazendo uso de (4.11) e 4.12), resulta

$$
\begin{aligned}
\int_{B}\left|\nabla u_{H}(x)\right|^{2} d x & =\int_{H \cap\{u \geq v\}}|\nabla u(x)|^{2} d x+\int_{H \cap\{u<v\}}\left|\nabla u\left(\sigma_{H}(x)\right)\right|^{2} d x \\
& +\int_{\sigma_{H}(H) \cap\{u<v\}}|\nabla u(x)|^{2} d x+\int_{\sigma_{H}(H) \cap\{u \geq v\}}\left|\nabla u\left(\sigma_{H}(x)\right)\right|^{2} d x \\
& =\int_{H \cap\{u \geq v\}}|\nabla u(x)|^{2} d x+\int_{\left.\left\{x \in \sigma_{H}(H) / u>v\right)\right\}}|\nabla u(x)|^{2} d x \\
& +\int_{\sigma_{H}(H) \cap\{u<v\}}|\nabla u(x)|^{2} d x+\int_{\left.\left\{x \in \sigma_{H}(H) / u \leq v\right)\right\}}|\nabla u(x)|^{2} d x \\
& =\int_{B}|\nabla u(x)|^{2} d x .
\end{aligned}
$$

Lema 4.8. [4, Lemma 2.2] Seja $F: B \times \mathbb{R} \rightarrow \mathbb{R}$ uma função de Carathéodory que satisfaz $F(x, 0)=0$ para todo $x \in B$ e considere

$$
\mathcal{M}_{F}:=\left\{u: B \rightarrow \mathbb{R} \text { mensurável }: \int_{B}|F(x, u(x))|: d x<\infty\right\}
$$

Além disso, suponha que existe $H \in \mathcal{H}$ tal que

$$
F(x, t)=F\left(\sigma_{H}(x), t\right) \quad x \in B \text { et } t \in \mathbb{R} .
$$

Então para todo $u \in \mathcal{M}_{F}$, tem-se

$$
\int_{B} F(x, u(x)) d x=\int_{B} F\left(x, u_{H}(x)\right) d x
$$

Demonstração. Sejam $u \in \mathcal{M}_{F}$ e $H \in \mathcal{H}$, usando a expressão (4.13), obtém-se 


$$
\begin{aligned}
\int_{B} F(x, u(x)) d x & =\int_{H} F(x, u(x)) d x+\int_{\mathbb{R}^{N} \backslash H} F(x, u(x)) d x \\
& =\int_{H}\left[F(x, u(x))+F\left(x, u\left(\sigma_{H}(x)\right)\right)\right] d x \\
& =\int_{H \cap\left\{u \geq u \circ \sigma_{H}\right\}}\left[F\left(x, u_{H}(x)\right)+F\left(x, u_{H}\left(\sigma_{H}(x)\right)\right)\right] d x \\
& +\int_{H \cap\left\{u<u \circ \sigma_{H}\right\}}\left[F\left(x, u_{H}\left(\sigma_{H}(x)\right)\right)+F\left(x, u_{H}(x)\right] d x\right. \\
& =\int_{H}\left[F\left(x, u_{H}(x)\right)+F\left(x, u_{H}\left(\sigma_{H}(x)\right)\right)\right] d x \\
& =\int_{B} F\left(x, u_{H}(x)\right) d x .
\end{aligned}
$$

Lema 4.9. Seja $\sigma_{H}$ a reflexão associada com algum meio espaço $H \in \mathcal{H}$ e $f: B \times \mathbb{R} \rightarrow \mathbb{R}$ uma função de Carathéodory (ver Definição A.27) tal que

$$
\begin{gathered}
f(x, t)=f\left(\sigma_{H}(x), t\right), \quad \forall x \in B, t \in \mathbb{R}, \\
t \rightarrow f(x, t) \text { é não decrescente sobre } \mathbb{R} \text { para todo } x \in B .
\end{gathered}
$$

Além disso, considere $u \in C^{2}(\bar{B})$ tal que $u_{H} \in C^{2}(\bar{B})$ e ambas funções são soluções clássicas da equação,

$$
-\Delta v=f(x, v) \text { sobre } B .
$$

Então uma das seguintes é verdade:

(i) $u(x)>u\left(\sigma_{H}(x)\right)$ para todo $x \in \operatorname{int}(H \cap B)$;

(ii) $u(x)<u\left(\sigma_{H}(x)\right)$ para todo $x \in \operatorname{int}(H \cap B)$;

(iii) $u(x)=u\left(\sigma_{H}(x)\right)$ para todo $x \in B$.

Demonstração. Para $x \in H \cap B$, tem-se $u_{H}(x)=\frac{1}{2}\left(u(x)+u\left(\sigma_{H}(x)\right)\right)+\frac{1}{2}\left|u(x)-u\left(\sigma_{H}(x)\right)\right|$. Assim,

$$
\left.\mid u-u \circ \sigma_{H}\right) \mid=2 u_{H}-\left(u+u \circ \sigma_{H}\right),
$$

e portanto $\left|u-u \circ \sigma_{H}\right| \in C^{2}(H \cap \bar{B})$. Por outro lado, desde que $\sigma_{H}(x) \in B$ e $u$ é solução da expressão 4.16 tem-se $-\Delta u\left(\sigma_{H}(x)\right)=f\left(\sigma_{H}(x), u\left(\sigma_{H}(x)\right)\right)$. Usando 4.14 e o fato que $-\Delta u\left(\sigma_{H}(x)\right)=-\Delta u \circ \sigma_{H}(x)$ (a prova de isto de forma geral está no Capitulo 3) 
resulta

$$
-\Delta u \circ \sigma_{H}(x)=f\left(x, u \circ \sigma_{H}(x)\right) \text {. }
$$

Logo, $u \circ \sigma_{H}$ é também solução de (4.16). Agora da hipótese e de (4.17), (4.18), (4.15) e lembrando que $u(x) \leq u_{H}(x)$, implica

$$
\begin{aligned}
-\Delta\left|u-u \circ \sigma_{H}\right| & =-2 \Delta u_{H}+\Delta\left(u+u \circ \sigma_{H}\right) \\
& =2 f\left(x, u_{H}(x)\right)-\left[f(x, u(x))+f\left(x, u \circ \sigma_{H}(x)\right)\right] \\
& =\left[f\left(x, u_{H}(x)-f(x, u(x))\right]+\left[f\left(x, u_{H}(x)-f\left(x, u \circ \sigma_{H}(x)\right)\right]\right.\right. \\
& \geq 0,
\end{aligned}
$$

para todo $x \in H \cap B$ e além disso $\left|u-u \circ \sigma_{H}\right|=0$ em $\partial(H \cap B)$. Portanto, pelo Principio do máximo, tem-se $u \equiv u \circ \sigma_{H}$ sobre $B$ ou $\left|u-u \circ \sigma_{H}\right|>0$ sobre $\operatorname{int}(H \cap B)$. Neste último obtém-se $u>u \circ \sigma_{H}$ ou $u<u \circ \sigma_{H}$ sobre $\operatorname{int}(H \cap B)$.

Teorema 4.10. Toda solução ground state u possui uma simetria de Schwarz folheada. Além disso, cada u é radial ou ué estritamente decrescente em $\theta \in(0, \pi)$ para $0<|x| \leq 1$.

Demonstração. Seja $u$ a solução ground state para o problema (2.1) quando $2<p<2^{*}$. Então pelo Teorema 2.16 tem-se $c_{J}=J(u)$.

Seja $H \in \mathcal{H}_{*}$, considerando $F(x, u)=|x|^{\alpha}|u|^{p}$, note que $F$ satisfaz as condições do Lema 4.8, então de isto e da Proposição 4.7 resulta

$$
\int_{B}\left|\nabla u_{H}(x)\right|^{2} d x=\int_{B}|\nabla u(x)|^{2} d x=\int_{B}|x|^{\alpha}|u|^{p} d x=\int_{B}|x|^{\alpha}\left|u_{H}\right|^{p} d x
$$

onde obtém-se ,

$$
J^{\prime}\left(u_{H}\right) u_{H}=0 \quad \text { e } \quad c_{J}=J(u)=J\left(u_{H}\right) .
$$

Observe que $u_{H} \in N_{J}$ e novamente usando o Teorema 2.16, resulta que $u_{H}$ é solução ground state para o problema (2.1) para $2<p<2^{*}$. Logo, $u, u_{H} \in C^{2}(\bar{B}) \cap C_{0}(\bar{B})$. Assim tem-se satisfeitas as condições do Lema 4.9 . Por outro lado, tome $x_{0} \in \operatorname{int}(B) \backslash\{0\}$ com

$$
u\left(x_{0}\right)=\max \left\{u(x): x \in B,|x|=\left|x_{0}\right|\right\},
$$

e $P=\frac{x_{0}}{\left|x_{0}\right|}$. Tendo em vista o Lema 4.9 , se fosse verdade o item (ii) então teríamos uma contradição com a maximalidade de $u\left(x_{0}\right)$. Assim, $u(x) \geq u\left(\sigma_{H}(x)\right)$ em $H \cap B$ para todo $H \in \mathcal{H}_{P}$, i.e., $u_{H}=u$ para todo $H \in \mathcal{H}_{P}$. Portanto pela Proposição 4.6 conclui-se 
que $u=u_{P}$, isto é, $u$ possui simetria de Schwarz folheada com respeito ao ponto $\mathrm{P}$ e escreve-se $u=u(r, \theta)$, onde $r=|x|$ e $\theta_{x}=\arccos \frac{x}{|x|} \cdot P$.

Agora vai ser mostrado que $u$ é radial ou $u(r, \theta)$ é estritamente decrescente em $\theta \in$ $(0, \pi)$ para $0<r \leq 1$. Lembra-se que não há meio espaço $H \in \mathcal{H}_{P}$ que satisfaz a condição $(i i)$ do Lema 4.9 .

Então, se a propriedade $(i)$ do Lema 4.9 for verdade para todo semi-espaço $H \in \mathcal{H}_{P}$, então escolhendo $x \in \operatorname{int}(H \cap B)$ e $y \in B \backslash\{0\}$ tais que $\theta_{x}<\theta_{y} \operatorname{com} \theta_{x}, \theta_{y} \in(0, \pi), 0<$ $r \leq 1$ e considerando $H$ tal que $x$ e $P \in H$ de forma que $y=\sigma_{H}(x)$ então obtémse $u\left(x, \theta_{x}\right)>u\left(y, \theta_{y}\right)$. Assim, $u(r, \theta)$ é estritamente decrescente em $\theta \in(0, \pi)$ para $0<r \leq 1$.

Se a proposição (iii) do Lema 4.9 for verdade para algum $H \in H_{P}$. Seja $0<\theta_{0}<$ $\pi / 2$ o ângulo formado por $P$ e hiperplano $\partial H_{0}$. Considerando $P_{0}=\sigma_{H_{0}}(P)$, tem-se $2 \theta_{0}=\arccos \left\langle P_{0} \cdot P\right\rangle$ e pelo item $(i i i), u(r P)=u\left(r P_{0}\right)$ para $0 \leq r \leq 1$. Desde que $u$ tem simetria de Schwarz folheada respeito a $P$, tem-se que $u$ é não crescente em o ângulo $\theta \in(0, \pi)$, então $u$ é constante sobre o arco formado pelo ângulo $2 \theta$, ie., $u(r, \theta)=u(r, 0)$, para todo $\theta \leq 2 \theta_{0}$. Logo, do Lema 4.9 deduz-se que $($ iii $)$ é verdade para todo $H \in \mathcal{H}_{P}$ no qual o ângulo entre $P$ e $H$ é menor que $2 \theta_{0}$. Note que pela simetria de Schwarz folheada obtém-se também que $u$ é constante sobre o arco formado pelo ângulo $4 \theta_{0}$. Portanto, $u(r, \theta)=u(r, 0)$ para todo $\theta \leq \min \left\{4 \theta_{0}, \pi\right\}$. De forma sucessiva para uma quantidade finita de casos, tem-se $u(r, \theta)=u(r, 0)$ para todo $\theta \leq \pi$. Portanto conclui-se que $u$ é radial. 


\section{Capítulo}

\section{5}

\section{Sobre a multiplicidade}

A existência, unicidade e multiplicidade de soluções positivas para a equação de Hénon dependem dos valores de $p$. No Capítulo 3 com $1<p<2$ e qualquer $\alpha>0$ foi provado que o problema tem única solução positiva e além disso é radial. Por outro lado, no Capítulo 4 foi mostrado que para $2<p<2^{*}$ e $\alpha$ suficientemente grande tem-se pelo menos duas soluções positivas. Neste capítulo apresentaremos resultados sobre a multiplicidade de soluções no caso em que $p>2$ incluindo caso $p \geq 2^{*}$.

Considere o funcional $Q_{\alpha}$ definido em $H_{0}^{1}(B)$ como segue

$$
Q_{\alpha}(u)=\frac{\int_{B}|\nabla u|^{2} d x}{\left(\int_{B}|x|^{\alpha}|u|^{p} d x\right)^{2 / p}} .
$$

Sejam $l \in \mathbb{N}$ tal que $2 \leq N-l \leq l$ e $N \geq 4$. Denota-se por $H_{l}(B)$ ao seguinte conjunto

$$
H_{l}(B)=\left\{u \in H_{0}^{1}(B) \mid u(y, z)=u(|y|,|z|) ;(y, z) \in B\right\}
$$

onde $y=\mathbb{R}^{l}, z \in \mathbb{R}^{N-l}$ e $x=(y, z)$. Note que $H_{l}(B)$ é um subconjunto de $H_{0}^{1}(B)$ e de funções que são radiais em $y$ e $z$. Considera-se o espaço $H_{l}$ munida da norma

$$
\|u\|=\left(\int_{B}|\nabla u|^{2} d x\right)^{1 / 2}, \quad u \in H_{l}(B) .
$$

Teorema 5.1. Seja $N \geq 4,2 \leq N-l \leq l$. Defina $p_{l}=\frac{2 l+2}{l-1}$ e suponha que $\alpha>\alpha_{0}(N)$. Então 
existe uma constante $C>0$ tal que para todo $u \in H_{l}(B)$ satisfaz,

$$
\left(\int_{B}|x|^{\alpha}|u|^{p_{l}} d x\right)^{1 / p_{l}} \leq C\left(\int_{B}|\nabla u|^{2} d x\right)^{1 / 2} .
$$

Demonstração. Veja [3, Theorem 2.1].

Corolário 5.2. Sob as mesmas hipóteses do Teorema 5.1.

i) Se $p \in\left[1, p_{l}\right]$. Então existe uma constante $C>0$ tal que

$$
\left(\int_{B}|x|^{\alpha}|u|^{p} d x\right)^{2 / p} \leq C \int_{B}|\nabla u|^{2} d x, \quad \forall u \in H_{l}(B) .
$$

ii) Se $p \in\left[1, p_{l}\right)$ e $\left(u_{n}\right)$ é uma sequência em $H_{l}(B)$ tal que $u_{n} \rightarrow 0$ em $H_{l}(B)$. Então

$$
\int_{B}|x|^{\alpha}\left|u_{n}\right|^{p} d x \longrightarrow 0
$$

\section{Demonstração.}

i) Seja $u \in H_{l}(B)$. Pela hipóteses tem-se que $|x|^{\frac{\alpha}{p_{l}}} u \in L^{p_{l}}(B)$ e desde que $1 \leq p \leq p_{l}$, fazendo uso da proposição B.3. obtém-se $|x|^{\frac{\alpha}{p_{l}}} u \in L^{p}$ e também existe $c>0$ tal que $\left.\left.|| x\right|^{\frac{\alpha}{p_{l}}} u\right|_{p} \leq\left.\left. c|| x\right|^{\frac{\alpha}{p_{l}}} u\right|_{p_{l}}$, equivalente

$$
\left(\int_{B}|x|^{\frac{\alpha}{p_{l}} p}|u|^{p} d x\right)^{1 / p} \leq c\left(\int_{B}|x|^{\alpha}|u|^{p_{l}} d x\right)^{1 / p_{l}} .
$$

Por outro lado, observe que $\frac{p}{p_{l}} \leq 1$ e assim $|x|^{\alpha} \leq\left(|x|^{\alpha}\right)^{p / p_{l}}$. Logo,

$$
\left(\int_{B}|x|^{\alpha}|u|^{p} d x\right)^{1 / p} \leq\left(\int_{B}|x|^{\frac{\alpha}{p_{l}} p}|u|^{p} d x\right)^{1 / p} .
$$

Das estimativas (5.1) - 5.2) e pelo Teorema 5.1, existirá $\tilde{c}>0$ tal que

$$
\left(\int_{B}|x|^{\alpha}|u|^{p} d x\right)^{1 / p} \leq c \tilde{c}\left(\int_{B}|\nabla u|^{2} d x\right)^{1 / 2} .
$$

Portanto,

$$
\left(\int_{B}|x|^{\alpha}|u|^{p} d x\right)^{2 / p} \leq(c \tilde{c})^{2} \int_{B}|\nabla u|^{2} d x .
$$


ii) Do item $(i)$ tem-se que a imersão $H_{l}(B) \hookrightarrow L^{p_{l}}\left(B,|x|^{\alpha}\right)$ é continua e desde que $H_{l}(B) \hookrightarrow H_{0}^{1}(B)$ é imersão contínua e $H_{0}^{1}(B) \hookrightarrow L^{1}\left(B,|x|^{\alpha}\right)$ é imersões compacta. Então pelo Teorema de interpolação (Teorema 2.8) tem-se que para $1 \leq p<p_{l}$, a imersão $H_{l}(B) \hookrightarrow L^{p}\left(B,|x|^{\alpha}\right)$ é compacta. Assim pela compacidade, desde que $\left(u_{n}\right) \rightarrow 0$ em $H_{l}(B)$, implica que $\left(u_{n}\right) \longrightarrow 0$ em $L^{p}\left(B,|x|^{\alpha}\right)$. Portanto

$$
\int_{B}|x|^{\alpha}\left|u_{n}\right|^{p} d x \longrightarrow 0
$$

Defina

$$
S_{\alpha, l}=\inf _{u \in H_{l} \backslash\{0\}} Q_{\alpha}(u) .
$$

As dois proposições seguintes mostram que $S_{\alpha, l}<S_{\alpha, p}^{\text {rad }}$. Para $\alpha$ suficientemente grande.

Proposição 5.3. [3. Proposition 3.3] Assuma $N \geq 4,2 \leq N-l \leq l$ e $p \in\left(2, \frac{2 l+2}{l-1}\right)$. Então existe uma constante $C>0$ tal que para todo a grande,

$$
S_{\alpha, p}^{\mathrm{rad}} \geq C \alpha^{1+\frac{2}{p}}
$$

Demonstração. No Lema 4.1 escolhemos $\beta=\frac{4 N}{\alpha+N}$, então

$$
\int_{B}|w|^{p}|x|^{\beta(\alpha+N)-N} d x=\int_{B}|w|^{p}|x|^{3 N} d x
$$

e desde que $\beta \leq 1$ (isto é quando $\alpha$ é grande), tem-se também

$$
\int_{B}|\nabla w|^{2}|x|^{(\beta-1)(N-2)} d x \geq \int_{B}|\nabla w|^{2} d x .
$$

Logo, para todo $w \in T_{\beta}\left(H_{0, \text { rad }}^{1}(B) \backslash\{0\}\right)$. Usando as duas estimativas anteriores e lembrando que $T_{\beta}\left(H_{0, \text { rad }}^{1}\right) \subseteq H_{0, \text { rad }}^{1}$, obtém-se

$$
\begin{aligned}
S_{\alpha, p}^{\mathrm{rad}} & =\inf _{\substack{w \in T_{\beta}\left(H_{0, \text { rad }}^{1}(B)\right) \\
w \neq 0}} \frac{1}{\beta^{1+2 / p}} \frac{\int_{B}|\nabla w|^{2}|x|^{(\beta-1)(N-2)} d x}{\left(\int_{B}|w|^{p}|x|^{\beta(\alpha+N)-N} d x\right)^{2 / p}} \\
& \geq \inf _{\substack{w \in T_{\beta}\left(H_{0, \text { rad }}^{1}\right) \\
w \neq 0}} \frac{1}{\beta^{1+2 / p}} \frac{\int_{B}|\nabla w|^{2} d x}{\left(\int_{B}|w|^{p}|x|^{3 N} d x\right)^{2 / p}} \\
& \geq \inf _{\substack{w \in H_{0, \text { rad }}^{1}(B) \\
w \neq 0}} \frac{1}{\beta^{1+2 / p}} \frac{\int_{B}|\nabla w|^{2} d x}{\left(\int_{B}|w|^{p}|x|^{3 N} d x\right)^{2 / p}}
\end{aligned}
$$




$$
\begin{aligned}
& =\frac{1}{\beta^{1+2 / p}} \inf _{\substack{w \in H_{0, \text { rad }}^{1}(B) \\
w \neq 0}} \frac{\int_{B}|\nabla w|^{2} d x}{\left(\int_{B}|w|^{p}|x|^{3 N} d x\right)^{2 / p}} \\
& =\frac{1}{\beta^{1+2 / p}} S_{3 N, p}^{r a d} .
\end{aligned}
$$

Note que $S_{3 N, p}^{\mathrm{rad}}$ está bem definida sempre que $p<\frac{2 N+2(3 N)}{N-2}$ e em nosso caso, isso é obtido desde que $p<\frac{2(l+1)}{l-1}$ e $2 \leq N-l \leq l$. Assim, para $\alpha$ suficientemente grande

$$
S_{\alpha, p}^{\mathrm{rad}} \geq C \frac{1}{\left(\frac{4 N}{\alpha+N}\right)^{1+2 / p}} \geq \tilde{C} \alpha^{1+2 / p}
$$

onde $\tilde{C}=\frac{C}{(4 N)^{1+2 / p}}$.

Proposição 5.4. [3, Proposition 3.4] Assuma $N \geq 4,2 \leq N-l \leq l$ e $p \in\left(2, \frac{2 l+2}{l-1}\right)$. Então existe uma constante $\tilde{C}>0$ tal que para todo $\alpha$ grande,

$$
S_{\alpha, l} \leq \tilde{C} \alpha^{(l+1-N)\left(1-\frac{2}{p}\right)+\frac{4}{p}}
$$

Demonstração.Vamos seguir a prova como foi feito em [3]. Sejam $x=(y, z) \in \mathbb{R}^{l} \times$ $\mathbb{R}^{N-l}, u \in H_{l}(B) \mathrm{e}$

$$
D=\left\{(s, t) \in \mathbb{R}^{2} / s, t \geq 0,0 \leq s^{2}+t^{2}<1\right\} .
$$

$$
\begin{aligned}
\text { Tem-se } u(x)=v(|y|,|z|) & =v(s, t) \mathrm{e} \\
\int_{B}|x|^{\alpha}|u|^{p} d x & =\omega_{N} \int_{D}\left(s^{2}+t^{2}\right)^{\alpha / 2}|v(s, t)|^{p} s^{l-1} t^{N-(l+1)} d s d t .
\end{aligned}
$$

Também,

$$
\int_{B}|\nabla u|^{2} d x=\omega_{N} \int_{D}|\nabla v(s, t)|^{2} s^{l-1} t^{N-l-1} d s d t .
$$

Defina

$$
\begin{gathered}
s=\rho \cos \theta, t=\rho \operatorname{sen} \theta \\
A=\left\{(\rho, \theta) \in \mathbb{R}^{2} / 0 \leq \rho<1,0 \leq \theta \leq \frac{\pi}{2}\right\}
\end{gathered}
$$

e

$$
w(\rho, \theta)=v(\rho \cos \theta, \rho \operatorname{sen} \theta)
$$


Fazendo mudança de variável, resulta

$$
\begin{aligned}
\int_{D}\left(s^{2}+t^{2}\right)^{\alpha / 2}|v(s, t)|^{p} s^{l-1} t^{N-(l+1)} d s d t & =\int_{A} \rho^{\alpha}|w(\rho, \theta)|^{p}(\rho \cos \theta)^{l-1}(\rho \operatorname{sen} \theta)^{N-l-1} \rho d \rho d \theta \\
& =\int_{A}|w(\rho, \theta)|^{p} \rho^{\alpha+N-1} H(\theta) d \rho \theta .
\end{aligned}
$$

e também,

$$
\begin{aligned}
\int_{D}|\nabla v(s, t)|^{2} s^{l-1} t^{N-l-1} d s d t & =\int_{A}\left(w_{\rho}^{2}(\rho, \theta)+\frac{1}{\rho^{2}} w_{\theta}^{2}(\rho, \theta)\right)(\rho \cos \theta)^{l-1}(\rho \operatorname{sen} \theta)^{N-l-1} \rho d \rho d \theta \\
& =\int_{A}\left(w_{\rho}^{2}(\rho, \theta)+\frac{1}{\rho^{2}} w_{\theta}^{2}(\rho, \theta)\right) \rho^{N-1} H(\theta) d \rho d \theta
\end{aligned}
$$

onde $H(\theta)=(\cos \theta)^{l-1}(\operatorname{sen} \theta)^{N-l-1}$.

Logo, substituindo as duas últimas igualdades nas estimativas (5.3) e (5.4), obtémse para cada $u \in H_{l}(B)$

$$
\frac{\int_{B}|\nabla u|^{2} d x}{\left(\int_{B}|x|^{\alpha}|u|^{p} d x\right)^{2 / p}}=\frac{\int_{A}\left(w_{\rho}^{2}(\rho, \theta)+\frac{1}{\rho^{2}} w_{\theta}^{2}(\rho, \theta)\right) \rho^{N-1} H(\theta) d \rho d \theta}{\left(\int_{A}|w(\rho, \theta)|^{p} \rho^{\alpha+N-1} H(\theta) d \rho \theta\right)^{2 / p}} .
$$

Defina $\tilde{A}=\left(\frac{1}{4}, \frac{3}{4}\right) \times\left(\theta_{1}, \theta_{2}\right) \operatorname{com} 0<\theta_{1}<\theta_{2}<\frac{\pi}{2}$ e para a função $\psi \in C_{0}^{\infty}(\tilde{A})$ e $\varepsilon>0$ fixado, defina também

$$
w^{\varepsilon}(\rho, \theta)=\psi\left(\rho^{\frac{1}{\varepsilon}}, \frac{\theta}{3}\right)
$$

Seja $\tilde{A}_{\varepsilon}=\left\{(\rho, \theta) \in \mathbb{R}^{2} /\left(\frac{1}{4}\right)^{\varepsilon}<\rho<\left(\frac{3}{4}\right)^{\varepsilon}, \varepsilon \theta_{1}<\theta<\varepsilon \theta_{2}\right\}$, note que $w^{\varepsilon} C_{0}^{\infty}\left(\tilde{A}_{\varepsilon}\right)$. Defina $v^{\varepsilon}(\rho \cos \theta, \rho \operatorname{sen} \theta)=w^{\varepsilon}(\rho, \theta)$, é claro que para cada $x \in \mathbb{R}^{l} \times \mathbb{R}^{N-l}$, $u^{\varepsilon}=v^{\varepsilon}(\rho \cos \theta, \rho \operatorname{sen} \theta)$ assim definindo pertence a $C_{0}^{\infty} \cap H_{l}(B)$.

Logo, para $u^{\varepsilon} \in H_{l}(B)$ em (5.5), resulta

$$
\frac{\int_{B}\left|\nabla u^{\varepsilon}\right|^{2} d x}{\left(\int_{B}|x|^{\alpha}\left|u^{\varepsilon}\right|^{p} d x\right)^{2 / p}}=\frac{\int_{A}\left(\left(w_{\rho}^{\varepsilon}\right)^{2}(\rho, \theta)+\frac{1}{\rho^{2}}\left(w_{\theta}^{\varepsilon}\right)^{2}(\rho, \theta)\right) \rho^{N-1} H(\theta) d \rho d \theta}{\left(\int_{A}\left|w^{\varepsilon}(\rho, \theta)\right|^{p} \rho^{\alpha+N-1} H(\theta) d \rho d \theta\right)^{2 / p}} .
$$

Fazendo mudança de variável $r=\rho^{\frac{1}{\varepsilon}}, \varphi=\frac{\theta}{3}$, tem-se $w^{\varepsilon}(\rho, \theta)=\psi\left(\rho^{\frac{1}{\varepsilon}}, \frac{\theta}{3}\right)$. Então

$$
w_{\rho}^{\varepsilon}(\rho, \theta)=\frac{1}{\varepsilon} \psi_{1}\left(\rho^{\frac{1}{\varepsilon}}, \theta / \varepsilon\right) \rho^{\frac{1}{\varepsilon}-1} \quad \text { e } \quad w_{\theta}^{\varepsilon}(\rho, \theta)=\frac{1}{\varepsilon} \psi_{1}\left(\rho^{\frac{1}{\varepsilon}}, \theta / \varepsilon\right)
$$


Logo obtém-se

$$
\begin{aligned}
\int_{A}\left(\left(w_{\rho}^{\varepsilon}\right)^{2}+\right. & \left.\frac{1}{\rho^{2}}\left(w_{\theta}^{\varepsilon}\right)^{2}\right) \rho^{N-1} H(\theta) d \rho d \theta \\
& =\int_{\tilde{A}_{\varepsilon}}\left(\frac{1}{\varepsilon^{2} \rho^{2-\frac{2}{\varepsilon}}} \psi_{1}^{2}\left(\rho^{\frac{1}{\varepsilon}}, \theta / \varepsilon\right)+\frac{1}{\varepsilon \rho^{2}} \psi_{2}^{2}\left(\rho^{\frac{1}{\varepsilon}}, \theta / \varepsilon\right)\right) \rho^{N-1} H(\theta) d \rho d \theta \\
= & \int_{\tilde{A}_{\varepsilon}}\left(\frac{1}{\varepsilon^{2} r^{2 \varepsilon-2}} \psi_{1}^{2}(r, \varphi)+\frac{1}{\varepsilon^{2} r^{2 \varepsilon}} \psi_{2}^{2}(r, \varphi)\right) r^{\varepsilon(N-1)} H(\varepsilon \theta) \varepsilon^{2} r^{\varepsilon-1} d r d \varphi \\
& =\int_{\tilde{A}_{\varepsilon}}\left(\psi_{1}^{2}+\frac{1}{r^{2}} \psi_{2}^{2}\right) r^{(\varepsilon-1)(N-2)} r^{N-1} H(\varepsilon \theta) d r d \varphi
\end{aligned}
$$

e

$$
\begin{aligned}
\int_{A}\left|w^{\varepsilon}(\rho, \theta)\right|^{p} \rho^{\alpha+N-1} H(\theta) d \rho d \theta & =\int_{\tilde{A}}\left|w^{\varepsilon}(\rho, \theta)\right|^{p} \rho^{\alpha+N-1} H(\theta) d \rho d \theta \\
& =\int_{\tilde{A}}\left|w^{\varepsilon}\right|^{p} \rho^{\alpha+N-1} H(\theta) d \rho d \theta \\
& =\int_{\tilde{A}}|\psi(r, \varphi)|^{p} r^{\varepsilon(\alpha+N-1)} H(\varepsilon \varphi) \varepsilon^{2} r^{\varepsilon-1} d r d \varphi \\
& =\varepsilon^{2} \int_{\tilde{A}}|\psi|^{p} r^{\varepsilon(\alpha+N)-1} H(\varepsilon \varphi) d r d \varphi
\end{aligned}
$$

Agora escolhendo $\varepsilon=\frac{N}{\alpha+N}$ e substituindo as igualdades (5.7) e 5.8) em (5.6), tem-se

$$
\frac{\int_{B}\left|\nabla u^{\varepsilon}\right|^{2} d x}{\left(\int_{B}|x|^{\alpha}\left|u^{\varepsilon}\right|^{p} d x\right)^{2 / p}}=\omega_{N} \frac{\int_{\tilde{A}_{\varepsilon}}\left(\psi_{1}^{2}+\frac{1}{r^{2}} \psi_{2}^{2}\right) r^{(\varepsilon-1)(N-2)} r^{N-1} H(\varepsilon \theta) d r d \varphi}{\varepsilon^{4 / p}\left(\int_{\tilde{A}}|\psi|^{p} r^{N-1} H(\varepsilon \varphi) d r d \varphi\right)^{2 / p}} .
$$

Pela definição de $H$ existem constantes $\bar{c}_{\varepsilon}, \tilde{c}_{\varepsilon}>0$ tais que $\bar{c} \varepsilon^{N-l-1} \leq H(\varepsilon \varphi) \leq \tilde{c}_{\varepsilon} \varepsilon^{N-l-1}$.

Portanto da ultima igualdade e lembrando que $\frac{1}{4} \leq r \leq \frac{3}{4}$, resulta

$$
\begin{aligned}
Q_{\alpha}\left(u^{\varepsilon}\right) & \leq \omega_{N} C \frac{\varepsilon^{(N-l-1)} \int_{\tilde{A}}\left(\psi_{1}^{2}+\frac{1}{r^{2}} \psi_{2}^{2}\right) r^{(\varepsilon-1)(N-2)} r^{N-1} d r d \varphi}{\varepsilon^{(N-l-1) \frac{2}{p}+\frac{4}{p}}\left(\int_{\tilde{A}}|\psi|^{p} r^{N-1} d r d \varphi\right)^{2 / p}} \\
& \leq \omega_{N} C \varepsilon^{(N-l-1)\left(1-\frac{2}{p}\right)-\frac{4}{p}} \frac{\int_{\tilde{A}}\left(\psi_{1}^{2}+\frac{1}{r^{2}} \psi_{2}^{2}\right) r^{(\varepsilon-1)(N-2)} r^{N-1} d r d \varphi}{\left(\int_{\tilde{A}}|\psi|^{p} r^{N-1} d r d \varphi\right)^{2 / p}}
\end{aligned}
$$


Logo,

$$
Q_{\alpha}\left(u^{\varepsilon}\right) \leq c \varepsilon^{(N-l-1)\left(1-\frac{2}{p}\right)-\frac{4}{p}}
$$

O que acarreta em,

$$
\begin{aligned}
S_{\alpha, l} & \leq c \varepsilon^{(N-l-1)\left(1-\frac{2}{p}\right)-\frac{4}{p}} \\
& =c\left(\frac{N}{\alpha+N}\right)^{(N-l-1)\left(1-\frac{2}{p}\right)-\frac{4}{p}} \\
& \leq c\left(\frac{N}{\alpha}\right)^{(N-l-1)\left(1-\frac{2}{p}\right)-\frac{4}{p}} \\
& =\tilde{c} \alpha^{(l+1-N)\left(1-\frac{2}{p}\right)+\frac{4}{p}}
\end{aligned}
$$

onde $\tilde{c}$ não depende de $\alpha$.

Teorema 5.5. [3. Proposition 3.5] Suponha $N \geq 4,2 \leq N-l \leq l e \quad p \in\left(2, \frac{2 l+2}{l-1}\right)$. Então para todo a grande,

$$
S_{\alpha, l}<S_{\alpha, p}^{\mathrm{rad}}
$$

Demonstração. Das proposições 5.4 e 5.3, obtém-se para $\alpha$ grande,

$$
S_{\alpha, l} \leq \bar{C} \alpha^{(l+1-N)\left(1-\frac{2}{p}\right)+\frac{4}{p}} \quad \text { e } \quad \tilde{C} \alpha^{1+2 / p} \leq S_{\alpha, p}^{\mathrm{rad}}
$$

onde $\tilde{C}, \bar{C}>0$. Além disso, note que pela hipótese tem-se $(l+1-N) \leq 1$ e $p>2$, de isto segue $(l+1-N)\left(1-\frac{2}{p}\right)+\frac{4}{p}<1+\frac{2}{p}$. Assim, para $\alpha$ grande resulta

$$
\tilde{C} \alpha^{(l+1-n)\left(1-\frac{2}{p}\right)+\frac{4}{p}}<\bar{C} \alpha^{1+2 / p} .
$$

Portanto o teorema está provado.

Lema 5.6. Seja $N \geq 4,2 \leq l_{1} \leq N-l_{1} e \quad 2 \leq l_{2} \leq N-l_{2}$, com $l_{1} \neq l_{2}$. Então $H_{l_{1}} \cap H_{l_{2}}=H_{0, \mathrm{rad}}^{1}(B)$.

Demonstração. Veja [21, Lemma 3.3].

Teorema 5.7. [3. Theorem 1.1] Assuma $N \geq 4$ e $p \in\left(2, \frac{2 N-2}{N-3}\right)$. Então, para todo a grande, o problema (2.1) tem pelo menos $\left[\frac{N}{2}\right]-1$ diferentes soluções não-radiais.

Demonstração. Sejam $l \in \mathbb{N}$ tal que $2 \leq N-l \leq l, p \in\left(2, p_{l}\right)$ e $\alpha>\alpha_{0}(N)$. Considere

$$
S_{\alpha, l}=\inf _{u \in H_{l} \backslash\{0\}} Q_{\alpha}(u)
$$


Note que do Corolário 5.2 tem-se $\frac{1}{c}<Q_{\alpha}(u)$ para todo $u \in H_{l}(B)$, então $0<S_{\alpha, l}$ e observe que $S_{\alpha, l}$ é atingido por algum não trivial $u_{l} \in H_{l}$, isto é pela consequência imediata do fato que $H_{l}(B) \hookrightarrow L^{p}\left(|x|^{\alpha}, B\right)$ é uma imersão continua e compacta para $1 \leq p<p_{l}$. Logo de forma similar ao que foi feito no Lema 2.14 item $(i v)$ pode-se dizer que existe $\lambda>0$ tal que $v_{l}=\lambda u_{l}$ é solução do problema (2.1) quando $2<p<\frac{2 N-2}{N-3} \mathrm{e}$ $N \geq 4$. Além disso, da hipótese $2 \leq N-l \leq l$, note que $\frac{N}{2} \leq l \leq N-2$ e fazendo estimativas algebraicas tem-se que existe $\left[\frac{N}{2}\right]-1$ inteiros $l$ que satisfaz $2 \leq N-l \leq l$. Note também que $\frac{2 N-2}{N-3} \leq \frac{2 l+2}{l-1}$ sempre que $2 \leq N-l$ e $N \geq 4$. Então segue que $p \in\left(2, \frac{2 l+2}{l-1}\right)$.

Agora pela Proposição 5.5 para todo $\alpha$ grande, $v_{l}$ é solução não radial , pois

$$
Q_{\alpha}\left(v_{l}\right)=Q_{\alpha}\left(u_{l}\right)=S_{\alpha, l}<S_{\alpha, p}^{\mathrm{rad}}=\inf _{\substack{u \in H_{0, r a d}^{1}(B) \\ u \neq 0}} Q_{\alpha}(u) .
$$

Por outro lado, seja $l_{1}, l_{2} \in \mathbb{N}$ tal que $2 \leq N-l_{1} \leq l_{1}$ e $2 \leq N-l_{2} \leq l_{2}$ com $u_{l_{1}} \in H_{l_{1}}$ e $u_{l_{2}} \in H_{l_{2}}$, fazendo uso do Lema 5.6, se suponhamos que $u_{l_{1}} \in H_{l_{2}}$, então teríamos $u_{l_{1}} \in H_{0, \text { rad }}^{1}(B)$ o qual é uma contradição, o mesmo acontece se $u_{l_{2}} \in H_{l_{1}}$. Portanto tem-se para cada $l \in \mathbb{N}$ que satisfaz $2 \leq N-l \leq l$, $v_{l}$ pertence só a $H_{l}$ e é solução não radial. Além disso, encontramos $\left[\frac{N}{2}\right]-1$ soluções não radiais para o problema (2.1) no caso $2<p<\frac{2 N-2}{N-3}$. 


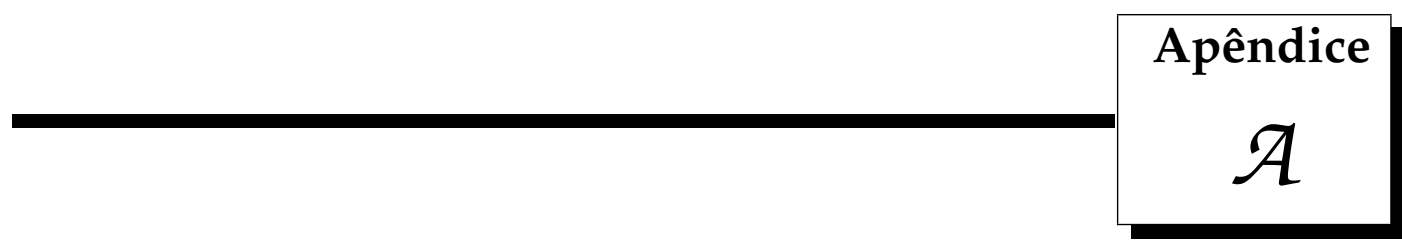

\section{Resultados Básicos}

Neste apêndice são apresentados resultados preliminares utilizados no desenvolvimento deste trabalho. Embora sejam omitidas as demonstração desses resultados, são citadas referências que as contém.

Definição A.1. Seja u uma função de clase $C^{2}(\Omega)$. O Laplaciano de u, denotado por $\Delta$ u, é o operador diferencial definido por

$$
\Delta u=\sum_{i=1}^{n} \frac{\partial^{2} u}{\partial x_{i}^{2}}=\operatorname{div} \nabla u .
$$

Teorema A.2. (Desigualdade de Hölder) [8, Theorem 4.6] Sejam $\Omega$ um domínio de $\mathbb{R}^{n}$, $1 \leq p \leq \infty$ e $1 \leq q \leq \infty \operatorname{com} \frac{1}{p}+\frac{1}{q}=1$. Se $f \in L^{p}(\Omega)$ e $g \in L^{q}(\Omega)$, então

$$
f g \in L^{1}(\Omega) \quad e \quad \int_{\Omega}|f g| d x \leq|f|_{p}|g|_{q}
$$

Teorema A.3. (Teorema do Divergente) [16, pag. 6] Sejam $\Omega$ um dominio limitado cuja fronteira é uma hiperfície de classe $C^{1}$ e $\eta$ o vetor normal unitário exterior à fronteira de $\Omega$. Para qualquer função $W: \Omega \longrightarrow \mathbb{R}^{n}, \operatorname{com} W \in C^{0}(\bar{\Omega}) \bigcap C^{1}(\Omega)$, tem-se

$$
\int_{\Omega} \operatorname{div} W d V=\int_{\partial \Omega} W \cdot \eta d S
$$

Corolário A.4. (Integração por Partes) Sejam $\Omega$ um dominio limitado cuja $\partial \Omega$ é uma hiperfície de classe $C^{1}$ e $G$ uma função de classe $C^{0}(\bar{\Omega}) \cap C^{1}(\Omega)$, então

$$
\int_{\Omega} u \frac{\partial G}{\partial x_{i}} d x=-\int_{\Omega} G \frac{\partial u}{\partial x_{i}} d x
$$


para toda função $u \in C^{1}(\Omega)$.

Demonstração. Basta aplicar o teorema do divergente ao campo $W=(0, \ldots 0, u G, 0, \ldots 0)$, onde $u G$ é a i-ésima coordenada de $W$.

Teorema A.5. (Teorema dos Multiplicadores de Lagrange) [20, Proposition 14.3] Sejam $E$ um espaço de Banach e $J, F \in C^{1}(E, \mathbb{R})$. Se $J$ é limitado inferiormente no conjunto $M=\{u \in M ; F(u)=0\}$ e valem as propiedades:

i) $F^{\prime}(u) \neq 0$, para todo $u \in M$,

ii) Existe $u_{0} \in M$ tal que $J\left(u_{0}\right)=\min _{u \in M} J(u)$.

Então, existe $\lambda \in \mathbb{R}$ tal que

$$
J^{\prime}\left(u_{0}\right)=\lambda F^{\prime}\left(u_{0}\right) .
$$

O número $\lambda \in \mathbb{R}$ é chamado multiplicador de Lagrange.

Teorema A.6. (Teorema de Lax-Milgram) [8, Corollary 5.8] Sejam H um espaço de Hilbert e a $: H \times H \rightarrow \mathbb{R}$ uma forma bilinear continua e coerciva. Se $\phi \in H^{*}$, então existe um único $u \in H$ tal que

$$
\phi(v)=a(u, v), \quad \text { para todo } v \in H
$$

Teorema A.7. (Teorema de Kakutani) [8. Theorem 3.17] Seja E um espaço de Banach. Então E é reflexivo se e somente se o conjunto $\bar{B}_{E}=\{x \in E ;\|x\| \leq 1\}$ é compacto na topologia fraca $\sigma\left(E, E^{*}\right)$.

Teorema A.8. (Teorema da Convergência Dominada de Lebesgue) [8, Theorem 4.2] Seja $\left(f_{n}\right)$ uma sequência de funções em $L^{1}(\Omega)$ que satisfaz:

(i) $f_{n}(x) \longrightarrow f(x)$ q.t.pem $\Omega$,

(ii) Existe uma função $h \in L^{1}(\Omega)$, tal que para todo $n \in \mathbb{N}$, verifica-se $\left|f_{n}(x)\right| \leq h(x)$ q.t.p em $\Omega$.

Então,

$$
f \in L^{1}(\Omega) \quad e \quad\left|f_{n}-f\right|_{1} \longrightarrow 0 .
$$

Teorema A.9. [8, Theorem 4.9] Sejam $1 \leq p \leq \infty,\left(u_{n}\right)$ uma sequência em $L^{p}(\Omega)$ e u $\in L^{p}(\Omega)$, tais que $u_{n} \longrightarrow u$ em $L^{p}(\Omega)$. Então, existe uma subsequência $\left(u_{n_{i}}\right) \subseteq\left(u_{n}\right)$ e $h \in L^{p}(\Omega)$, satisfazendo:

(i) $u_{n_{i}}(x) \longrightarrow u(x)$ q.t.p em $\Omega$, 
(ii) $\left|u_{n_{i}}(x)\right| \leq h(x)$ para todo $i \in \mathbb{N}$.

Lema A.10. (Lema de Hopf) [15, pag. 330] Seja $v \in C^{2}(\Omega) \cap C^{1}(\bar{\Omega})$ e suponha que

$$
-\Delta v \geq 0 \quad \text { em } \Omega
$$

e que existe um ponto $x_{0} \in \partial \Omega$ tal que

$$
v\left(x_{0}\right)<v(x) \quad \forall x \in \Omega
$$

Suponha também que $\Omega$ satisfaz a condição de bola interior em $x_{0}$, isto é, existe uma bola aberta $B^{\prime} \subseteq \Omega \operatorname{com} x_{0} \in \partial B^{\prime}$. Então

$$
\frac{\partial v}{\partial \eta}\left(x_{0}\right)<0
$$

onde $\eta$ é vetor unitário normal à $\partial B^{\prime}$ em $x_{0}$.

Lema A.11. [14, Lemma 5.3] Seja $w, \tilde{w} \in C^{2}(\Omega) \cap C^{1}(\bar{\Omega})$ tais que $w, \tilde{w}>0$ em $\Omega, w, \tilde{w} \equiv 0$ sobre $\partial \Omega$ e $\frac{\partial w}{\partial \eta}, \frac{\partial \tilde{w}}{\partial \eta}<0$ sobre $\partial \Omega$. Então para $\tau>0$ suficientemente pequeno, $w>\tau \tilde{w}$ em $\Omega$.

Teorema A.12. (Principio do Máximo Forte) [15, pag. 350] Seja $\Omega$ um domínio limitado regular de $\mathbb{R}^{n}$ e $u \in C^{2}(\Omega) \cap C(\bar{\Omega})$, com $u \neq \equiv$ em $\Omega$ e tal que

$$
-\Delta u \geq 0 \quad \text { em } \quad \Omega \quad \text { e } \quad u=0 \text { sobre } \partial \Omega \text {. }
$$

Então $u>0$ em $\Omega$.

\section{A.1 Os Espações de Sobolev $W^{1, p}(I)$ e $W^{k, p}(\Omega)$}

Definição A.13. Sejam $I=(a, b)$ um intervalo aberto, possivelmente ilimitado e $p \in[1, \infty]$. O espaço de Sobolev $W^{1, p}(I)$ é definido por

$$
W^{1, p}(I)=\left\{u \in L^{p}(I) ; \exists g \in L^{p}(I) \text { tal que } \int_{I} u \varphi^{\prime} d t=-\int_{I} g \varphi d t, \forall \varphi \in C_{c}^{1}(I)\right\} .
$$

Também usaremos a notação $H^{1}(I)=W^{1,2}(I)$. Além disso, para $u \in W^{1, p}(I)$ denotamos por $u^{\prime}$ a função $g$ que satisfaz

$$
\int_{I} u \varphi^{\prime} d t=-\int_{I} g \varphi d t, \forall \varphi \in C_{c}^{1}(I)
$$


Observação A.14. Seja $u \in C^{1}(I) \cap L^{p}(I)$ tal que $u^{\prime} \in L^{p}(I)$; aqui $u^{\prime}$ representa a derivada clássica de $u$. Então $u \in W^{1, p}(I)$ e além disso, a derivada clássica de u coincide com a derivada de u no sentido $W^{1, p}(I)$.

Consideraremos o espaço $W^{1, p}(I)$ dotado com a norma,

$$
\|u\|_{1, p}=\left(|u|_{p}^{p}+\left|u^{\prime}\right|_{p}^{p}\right)^{\frac{1}{p}} ; \quad 1 \leq p<\infty .
$$

Definição A.15. Sejam $u, v \in L_{L o c}^{1}(\Omega)$ e $\alpha=\left(\alpha_{1}, \alpha_{2}, \ldots, \alpha_{n}\right)$ um multi-índice de ordem $|\alpha|=\alpha_{1}+\ldots+\alpha_{n}$. Dizemos que v é $\alpha$-derivada fraca de u e escrevemos $D^{\alpha} u=v$, desde que

$$
\int_{\Omega} u D^{\alpha} \varphi d x=(-1)^{|\alpha|} \int_{\Omega} v \varphi d x, \quad \forall \varphi \in C_{c}^{\infty}(\Omega)
$$

$\operatorname{com} D^{\alpha} \varphi=\frac{\partial^{\alpha_{1}}}{\partial x_{1}^{\alpha_{1}}} \cdots \frac{\partial^{\alpha_{n}}}{\partial x_{n}^{\alpha_{n}}} \varphi$

Definição A.16. Sejam $p \in[1, \infty], k$ um inteiro positivo e $\Omega$ um aberto não vazio de $\mathbb{R}^{n}$. Definimos o espaço de Sobolev $W^{k, p}(\Omega)$ como

$$
W^{k, p}(\Omega)=\left\{u \in L^{p}(\Omega) ; D^{\alpha} u \in L^{p}(\Omega), \quad \forall \alpha \in \mathbb{N}^{n} \operatorname{com}|\alpha| \leq k\right\}
$$

Observe que na definição de $W^{k, p}(\Omega)$ a derivada considerada é a derivada fraca. Assim, dizer que $D^{\alpha} u \in L^{p}(\Omega)$ significa que existe $v_{\alpha} \in L^{p}(\Omega)$ tal que $D^{\alpha} u=v_{\alpha}$ no sentido fraco.

Definição A.17. Se $u \in W^{k, p}(\Omega)$ definimos sua norma como sendo

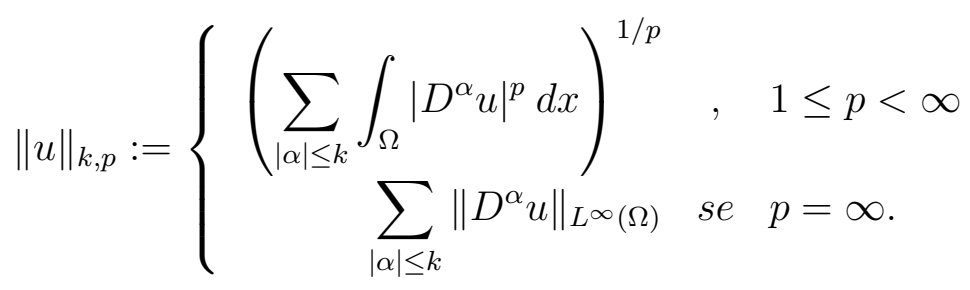

Se $p=2$, escreveremos

$$
H^{k}(\Omega)=W^{k, 2}(\Omega), \quad k=0,1,2, \ldots
$$

Definição A.18. Seja $B$ a bola unitária aberta em $\mathbb{R}^{n}$ centrada em zero. $W_{\text {rad }}^{1, p}(B)$ denota o espaço das funções em $W^{1, p}(B)$ que são radialmente simétricas. Também é usada a notação $H_{\text {rad }}^{1}(B)=W_{\text {rad }}^{1,2}(B)$. 
Teorema A.19. (Desigualdade geral de Sobolev) [1. Theorem 4.12] Seja $\Omega \subseteq \mathbb{R}^{n}$ um aberto limitado regular. Então as imersões abaixo são contínuas:

(i) Se $k p<n$ então $W^{k, p}(\Omega) \hookrightarrow L^{q}(\Omega)$ para todo $1 \leq q \leq \frac{p n}{n-k p}$.

(ii) Se $k p=$ n e $p>1$ então $W^{k, p}(\Omega) \hookrightarrow L^{q}(\Omega)$ para todo $1 \leq q<\infty$.

(iii) Se $k=n$ então $W^{n, 1}(\Omega) \hookrightarrow C^{0,1}(\bar{\Omega})$.

(iv) Se $k p>n$, então $W^{k, p}(\Omega) \hookrightarrow C^{k-\left[\frac{n}{p}\right]-1, \gamma}(\bar{\Omega})$, com

$$
\gamma= \begin{cases}{\left[\frac{n}{p}\right]+1-\frac{n}{p}, \quad \text { se }} & {\left[\frac{n}{p}\right] \quad \text { não é um inteiro, }} \\ \text { qualquer positivo } \quad<1, & \text { se } \quad\left[\frac{n}{p}\right] \text { é um inteiro. }\end{cases}
$$

Teorema A.20. (Imersões Compactas: Rellich-Kondrachov) [1, Theorem 6.3] Seja $\Omega \subseteq$ $\mathbb{R}^{n}$ um aberto limitado regular. Então, as seguintes imersões são compactas:

(i) Se $k p<n$ então $W^{k, p}(\Omega) \hookrightarrow L^{q}(\Omega)$ para todo $1 \leq q<\frac{p n}{n-k p}$.

(ii) Se $k p=$ n e $p>1$ então $W^{k, p}(\Omega) \hookrightarrow L^{q}(\Omega)$ para todo $1 \leq q<\infty$.

(iii) Se $k=$ n então $W^{n, 1}(\Omega) \hookrightarrow C^{0, \gamma}(\bar{\Omega})$ para todo $0 \leq \gamma<1$.

(iv) Se $k p>n$, então $W^{k, p}(\Omega) \hookrightarrow C^{k-\left[\frac{n}{p}\right]-1, \gamma}(\bar{\Omega})$, com

$$
0 \leq \gamma<\left\{\begin{array}{lll}
{\left[\frac{n}{p}\right]+1-\frac{n}{p},} & \text { se } & {\left[\frac{n}{p}\right] \quad \text { não é um inteiro, }} \\
\text { qualquer positivo } \quad<1, & \text { se } \quad\left[\frac{n}{p}\right] \quad \text { é um inteiro. }
\end{array}\right.
$$

\section{A.2 Resultados sobre existência de solução clássica}

Teorema A.21. [18, Theorem 9.15] Seja $\Omega$ um dominio limitado $C^{1,1} \mathrm{em} \mathbb{R}^{n}$. Se $f \in L^{p}(\Omega)$ e $\varphi \in W^{2, p}(\Omega), \operatorname{com} 1<p<\infty$, então o problema de Dirichlet $-\Delta u=f$ in $\Omega, u-\varphi \in W_{0}^{1, p}(\Omega)$ tem uma única solução forte $u \in W^{2, p}(\Omega)$, i.é. existe única $u \in W^{2, p}(\Omega)$ tal que $-\Delta u=f$ q.t.p. $\Omega$ e $u-\varphi \in W_{0}^{1, p}(\Omega)$.

Teorema A.22. [18. Corollary 4.14] Seja $0<\gamma<1 e B \subset \mathbb{R}^{n}$ a bola unitária aberta centrada em zero. Se $f \in C^{0, \alpha}(\bar{B})$ e $\varphi \in C^{2, \alpha}(\bar{B})$, então problema de Dirichlet, $-\Delta u=f$ em $B, u=\varphi$ sobre $\partial B$, tem uma única solução clássica $u \in C^{2, \alpha}(\bar{B})$. 


\section{A.3 Princípio geral minimax}

Teorema A.23. [28, Theorem 2.8] Seja E um espaço de Banach. Seja $M_{0}$ um subespaço fechado de um espaço métrico $M$ e $\Gamma_{0} \subset C\left(M_{0}, E\right)$. Define

$$
\Gamma_{0}:=\left\{\gamma \in C(M, E):\left.\gamma\right|_{M_{0}} \in \Gamma_{0}\right\}
$$

Se $\varphi \in C^{1}(E, \mathbb{R})$ satisfaz

$$
\infty>c=\inf _{\gamma \in \Gamma} \sup _{u \in M} \varphi(\gamma(u))>a:=\sup _{\gamma_{0} \in \Gamma_{0}} \sup _{u \in M_{0}} \varphi\left(\gamma_{0}(u)\right) .
$$

Então, para todo $\varepsilon \in(0,(c-a) / 2), \delta>0$ e $\gamma \in \Gamma$ tal que

$$
\sup _{M} \varphi \circ \gamma \leq c+\varepsilon
$$

existe $u \in E$ tal que

(a) $c-2 \varepsilon \leq \varphi(u) \leq c+2 \varepsilon$,

(b) $\operatorname{dist}(u, \gamma(M)) \leq 2 \delta$,

(c) $\left\|\varphi^{\prime}(u)\right\| \leq 8 \varepsilon / \delta$.

Definição A.24. (Brézis-Coron-Nirenberg, 1980) Seja E um espaço de Banach, $\varphi \in C^{1}(E, \mathbb{R})$ e $c \in \mathbb{R}$. O funcional $\varphi$ satisfaz a condição $(P S)_{c}$ se qualquer sequência $\left(u_{n}\right) \subseteq E$ tal que

$$
\varphi\left(u_{n}\right) \longrightarrow c, \quad \varphi^{\prime}\left(u_{n}\right) \longrightarrow 0
$$

possui uma subsequência convergente.

\section{A.4 O Teorema da Passo da Montanha}

Uma das principais ferramentas empregadas para estudar a existência de soluções de problemas elípticos semilineares é a teoria de pontos críticos. Um dos resultados principais desta teoria é o Teorema do Passo da Montanha de Ambrosetti e Rabinowitz [2]. Este teorema garante, sob hipóteses adequadas, a existência de pontos críticos minimax para funcionais $J: E \rightarrow \mathbb{R}$ onde $E$ é um espaço de Banach real. Para enunciar o Teorema do Passo da Montanha precisamos da seguinte noção de compacidade. 
Definição A.25. (Condição de Palais-Smale) Sejam E um espaço de Banach e $J \in C^{1}(E, \mathbb{R})$. Dizemos que J satisfaz a condição de Palais-Smale (PS), se toda sequência $\left(u_{n}\right)$ em E satisfazendo:

(i) $\left(J\left(u_{n}\right)\right)$ é limitada e

(ii) $J^{\prime}\left(u_{n}\right) \longrightarrow 0$ quando $n \longrightarrow \infty$,

possui uma subsequência convergente.

Teorema A.26. (Teorema do Passo da Montanha) [10, Pag. 28] Seja E um espaço de Banach e $J \in C^{1}(E, \mathbb{R})$ um funcional satisfazendo a condição (PS). Se e $\in E$ e $0<\rho<\|e\|$ são tais que

$$
a=\max \{J(0), J(e)\}<\inf _{\|u\|=\rho} J(u)=b
$$

então

$$
c=\inf _{\gamma \in \Gamma} \sup _{t \in[0,1]} J(\gamma(t))
$$

é um valor crítico de $J \operatorname{com} c \geq b$, onde $\Gamma$ é a classe de caminhos contínuos ligando 0 a e, isto é,

$$
\Gamma=\{\gamma \in C([0,1], E) ; \gamma(0)=0 \text { e } \gamma(1)=e\}
$$

\section{A.5 Operador de Nemytskii}

Seja $\Omega$ um subconjunto de $\mathbb{R}^{n}$ e $N \geq 1$.

Definição A.27. A função $f: \Omega \times \mathbb{R} \rightarrow \mathbb{R}$ é denominada função de Carathéodory se

(a) Para cada $s \in \mathbb{R}$ fixado, a função $x \mapsto f(x, s)$ é (Lebesgue) mensurável em $\Omega$.

(b) Para quase todo $x \in \Omega$ fixado, a função $s \mapsto f(x, s)$ é continua em $\mathbb{R}$.

Seja $\mathcal{M}$ conjunto das funções mensuráveis $u: \Omega \rightarrow \mathbb{R}$.

Teorema A.28. [11, Theorem 2.1] Se $f: \Omega \times \mathbb{R} \rightarrow \mathbb{R}$ é uma função de Carathéodory então a função $x \mapsto f(x, u(x))$ é mensurável para todo $u \in \mathcal{M}$.

Assim, para cada função de Carathéodory a Proposição A.28 permite definir o operador

$$
\begin{aligned}
& \mathcal{N}_{f}: \mathcal{M} \rightarrow \mathcal{M} \\
& u \mapsto \mathcal{N}_{f} u: \Omega \rightarrow \mathbb{R} \\
& x \mapsto\left(\mathcal{N}_{f} u\right)(x)=f(x, u(x)),
\end{aligned}
$$

o qual é chamado de operador de Nemytskii. 
Teorema A.29. [11, Theorem 2.3] Suponha que existam uma constante $c>0$, uma função $b(x) \in L^{q}(\Omega), 1 \leq q \leq \infty$ e $r>0$ tais que

$$
|f(x, s)| \leq c|s|^{r}+b(x), \quad \forall x \in \Omega, \quad \forall s \in \mathbb{R} .
$$

Então:

(i) O operador $\mathcal{N}_{f}: L^{q r}(\Omega) \rightarrow L^{q}(\Omega)$ está bem definido;

(ii) $\mathcal{N}_{f}$ é contínuo;

(iii) $\mathcal{N}_{f}$ é limitado, isto é, $\mathcal{N}_{f}(A)$ é limitado em $L^{q}(\Omega)$ para todo $A \subset L^{q r}(\Omega)$ limitado. 


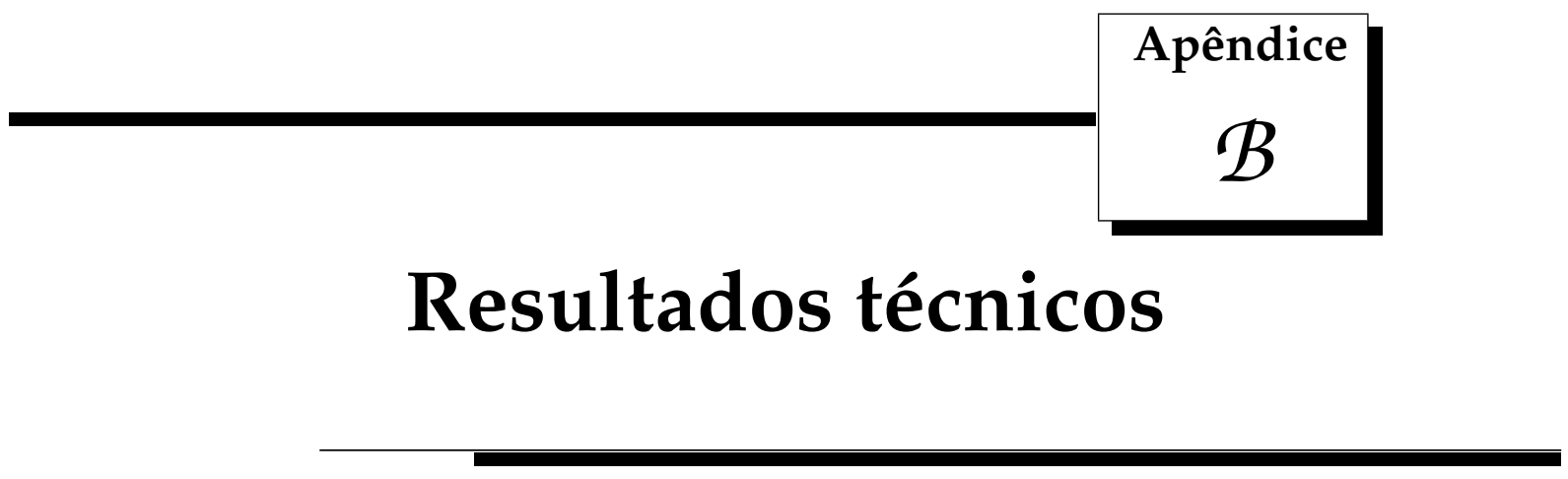

Nesta parte do apêndice estão apresentados alguns resultados técnicos utilizados nos capítulos desta dissertação.

Proposição B.1. Sejam $p \in \mathbb{R} \operatorname{com} 1 \leq p \leq 2^{*}$ e $M=\left\{u \in H_{0}^{1}(B) ; \int_{B}|x|^{\alpha}|u|^{p} d x=1\right\}$. Então,

$$
\inf _{\substack{u \in H_{0}^{1}(B) \\ u \neq 0}} \frac{\int_{B}|\nabla u|^{2} d x}{\left(\int_{B}|x|^{\alpha}|u|^{p} d x\right)^{\frac{2}{p}}}=\inf _{u \in M} \int_{B}|\nabla u|^{2} d x
$$

Demonstração. Sejam $u \in H_{0}^{1}(B)$ e $w=\frac{u}{\left(\int_{B}|x|^{\alpha}|u|^{p} d x\right)^{\frac{2}{p}}}$. Tem-se que

$$
\int_{B}|x|^{\alpha}|w|^{p} d x=1
$$

Assim $w \in M \mathrm{e}$

$$
\inf _{u \in M} \int_{B}|\nabla u|^{2} d x \leq \int_{B}|\nabla w|^{2} d x=\frac{\int_{B}|\nabla u|^{2} d x}{\left(\int_{B}|x|^{\alpha}|u|^{p} d x\right)^{\frac{2}{p}}} .
$$

Com isto,

$$
\inf _{u \in M} \int_{B}|\nabla u|^{2} d x \leq \inf _{\substack{u \in H_{0}^{1}(B) \\ u \neq 0}} \frac{\int_{B}|\nabla u|^{2} d x}{\left(\int_{B}|x|^{\alpha}|u|^{p} d x\right)^{\frac{2}{p}}} .
$$

Por outro lado, se $v \in M$ então

$$
\inf _{\substack{u \in H_{0}^{1}(B) \\ u \neq 0}} \frac{\int_{B}|\nabla u|^{2} d x}{\left(\int_{B}|x|^{\alpha}|u|^{p} d x\right)^{\frac{2}{p}}} \leq \frac{\int_{B}|\nabla v|^{2} d x}{\left(\int_{B}|x|^{\alpha}|v|^{p} d x\right)^{\frac{2}{p}}}=\int_{B}|\nabla v|^{2} d x .
$$


Assim,

$$
\inf _{\substack{u \in H_{0}^{1}(B) \\ u \neq 0}} \frac{\int_{B}|\nabla u|^{2} d x}{\left(\int_{B}|x|^{\alpha}|u|^{p} d x\right)^{\frac{2}{p}}} \leq \inf _{u \in M} \int_{B}|\nabla u|^{2} d x,
$$

as desigualdades (B.1) e (B.2) implicam

$$
\inf _{\substack{u \in H_{0}^{1}(B) \\ u \neq 0}} \frac{\int_{B}|\nabla u|^{2} d x}{\left(\int_{B}|x|^{\alpha}|u|^{p} d x\right)^{\frac{2}{p}}}=\inf _{u \in M} \int_{B}|\nabla u|^{2} d x .
$$

Portanto a proposição está demostrada.

Proposição B.2. Se $u \in H_{0}^{1}(B)$ então $\|u\|=\||u|\|$.

Demonstração. Sejam $u^{+}=\max \{u, 0\}$ e $u^{-}=-\min \{u, 0\}$. Então,

$$
u=u^{+}-u^{-}, \quad|u|=u^{+}+u^{-}, \quad \nabla u^{+}=\nabla u \cdot \mathcal{X}_{[u>0]} \quad \text { e } \quad \nabla u^{-}=\nabla u \cdot \mathcal{X}_{[u<0]} .
$$

De onde segue que,

$$
\begin{aligned}
\|u\|^{2} & =\left\langle u^{+}-u^{-}, u^{+}-u^{-}\right\rangle=\left\|u^{+}\right\|^{2}+\left\|u^{-}\right\|^{2}, \\
\|\| u \|^{2} & =\left\langle u^{+}+u^{-}, u^{+}+u^{-}\right\rangle=\left\|u^{+}\right\|^{2}+\left\|u^{-}\right\|^{2}
\end{aligned}
$$

de onde segue a igualdade desejada.

Proposição B.3. Se $\mu(\Omega)<\infty$ e $1 \leq r<p$. Então $L^{p}(\Omega) \subseteq L^{r}(\Omega)$ e existe $c>0$ tal que

$$
|u|_{r} \leq c|u|_{p} \quad \forall u \in L^{p}(\Omega)
$$

Demonstração. Seja $u \in L^{p}(\Omega)$. Então $|u|^{p} \in L^{1}(\Omega)$ e também como $|u|^{p}=\left(|u|^{r}\right)^{\frac{p}{r}}$ temos $|u|^{r} \in L^{\frac{p}{r}}$.

Seja $q$ o expoente conjugado de $\frac{p}{r}$. Como $1 \in L^{q}(\Omega)$, então pela desigualdade de Hölder, temos que $|u|^{r} \in L^{1}(\Omega)$ e

$$
\int_{\Omega}|u|^{r} d x \leq\left(\int_{\Omega} d x\right)^{\frac{1}{q}}\left(\int_{\Omega}\left(|u|^{r}\right)^{\frac{p}{r}} d x\right)^{\frac{r}{p}} .
$$

Assim,

$$
\left(\int_{\Omega}|u|^{r} d x\right)^{\frac{1}{r}} \leq c\left(\int_{\Omega}|u|^{p} d x\right)^{\frac{1}{p}}
$$


onde $c=(\mu(\Omega))^{\frac{1}{q r}}$.

Definição B.4. Seja $\varphi: U \rightarrow \mathbb{R}$ onde $U$ é um subconjunto aberto de um espaço de Banach $X$ e $u \in U$. Dizemos que $\varphi$ é Gâteaux-diferenciável em u se existe $A \in X^{*}$, tal que

$$
\lim _{t \rightarrow 0} \frac{\varphi(u+t v)-\varphi(u)}{t}=A v, \quad \forall v \in X
$$

O operador A é chamado a derivada de Gâteaux de $\varphi$ em u é é denotado por $\varphi^{\prime}(u)$.

Definição B.5. Seja $\varphi: U \rightarrow \mathbb{R}$ onde $U$ é um subconjunto aberto de um espaço de Banach $X$ e $u \in U$. Dizemos que $\varphi$ é Fréchet-diferenciável em u se existe $A \in X^{*}$, tal que

$$
\lim _{h \rightarrow 0} \frac{|\varphi(u+h)-\varphi(u)-A h|}{\|h\|}=0 .
$$

O operador A é chamado a derivada de Fréchet de $\varphi$ em u e é denotado por $D \varphi(u)$.

Observação: operador adjunto. Sejam $E$ e $F$ espaços normados e $T: E \rightarrow F$ um operador linear contínuo. O operador adjunto de $T$, representado por $T^{*}$ é o operador $T^{*}: F^{*} \rightarrow E^{*}$ definido por $T^{*} \phi:=\phi \circ T$ para $\phi \in F^{*}$. Tem-se então que $T^{*}$ é também um operador linear contínuo.

Proposição B.6. [28, Proposition 1.3] Suponha que $\varphi: U \rightarrow \mathbb{R}$ tenha derivada de Gâteaux em todo ponto de $U$ e que $\varphi^{\prime}: U \rightarrow X^{*}$ seja contínua (isto é, $\varphi$ tem derivada de Gâteaux contínua em $U)$. Então $\varphi$ é Fréchet-diferenciável em $U$ e $D \varphi=\varphi^{\prime}$. Em particular, $D \varphi: U \rightarrow X^{*}$ é contínua, isto é, $\varphi$ tem derivada de Fréchet contínua em $U$ (o que é denotado $\varphi \in C^{1}(U, \mathbb{R})$ ).

Lema B.7. Seja $p \in \mathbb{R}$ com $1<p<2^{*}$. O funcional energia $J: H_{0}^{1}(B) \rightarrow \mathbb{R}$ associado ao problema (2.1), definido por

$$
J(u)=\frac{1}{2} \int_{B}|\nabla u|^{2} d x-\frac{1}{p} \int_{B}|x|^{\alpha}|u|^{p} d x, \quad u \in H_{0}^{1}(B),
$$

está bem definido. Além disso, $J \in C^{1}\left(H_{0}^{1}(B), \mathbb{R}\right)$ com

$$
J^{\prime}(u) v=\int_{B} \nabla u \cdot \nabla v d x-\int_{B}|x|^{\alpha}|u|^{p-2} u v d x, \quad \forall u, v \in H_{0}^{1}(B) .
$$

Demonstração. Considere em $H_{0}^{1}(B)$ a norma definida por $\|u\|:=\left(\int_{B}|\nabla u|^{2} d x\right)^{1 / 2}$. Esta norma é equivalente à norma $\left(\|u\|_{2}^{2}+\|\nabla u\|_{2}^{2}\right)^{1 / 2}$ devido a desigualdade de Poincaré. Tem-se

$$
J(u)=L(u)-I(u),
$$


onde $L(u)=\frac{1}{2}\|u\|^{2}$ e $I(u)=\frac{1}{p} \int_{B}|x|^{\alpha}|u|^{p} d x$. Para provar que o funcional $J$ está bem definido, basta verificar que o funcional $I$ está bem definido. Para isto, desde que $\alpha>0$, e fazendo uso da imersão de Sobolev $H_{0}^{1}(B) \hookrightarrow L^{p}(B)$, segue-se que

$$
\int_{B}|x|^{\alpha}|u|^{p} d x \leq \int_{B}|u|^{p} d x<\infty
$$

Portanto o funcional $J$ está bem definido.

Agora resta mostrar que $J \in C^{1}\left(H_{0}^{1}(B)\right)$. Para isto faremos uso da Proposição B.6. O primeiro passo é mostrar que $J$ é Gâteaux-diferenciável em $u \in H_{0}^{1}(B)$. De fato, a prova é feita de forma separada para cada funcional $I$ e $L$ respectivamente.

Sejam $u, v \in H_{0}^{1}(B)$. Então

$$
\frac{I(u+t v)-I(u)}{t}=\frac{1}{p} \int_{B}|x|^{\alpha}\left(\frac{|u+t v|^{p}-|u|^{p}}{t}\right) d x .
$$

Seja $g: \mathbb{R} \rightarrow \mathbb{R}$, definida por $g(s)=|s|^{p}$, então $g^{\prime}(s)=p s|s|^{p-2}$. Pelo Teorema do Valor Médio na reta, para cada $x \in B$ existe $\vartheta_{x}$ entre $u(x)$ e $u(x)+t v(x)$ tal que

$$
g(u(x)+t v(x))-g(u(x))=g^{\prime}\left(\vartheta_{x}\right) t v(x) .
$$

Logo usando isto, tem-se

$$
|x|^{\alpha} \frac{|u(x)+t v(x)|^{p}-|u(x)|^{p}}{t}=p|x|^{\alpha} \vartheta_{x}\left|\vartheta_{x}\right|^{p-2} v(x) \longrightarrow p|x|^{\alpha} u(x)|u(x)|^{p-2} v(x),
$$

q.t.p. $x \in B$ quando $t \longrightarrow 0$. Por outro lado, para $0<|t|<1$,

$$
\left|\vartheta_{x}\right|<|u(x)|+|u(x)+t v(x)| \leq 2|u(x)|+|t||v(x)| \leq 2|u(x)|+|v(x)|,
$$

e portanto

$$
\left|\vartheta_{x}\right|^{p-1} \leq(2|u(x)|+|v(x)|)^{p-1} \leq 4^{p-1}\left(|u(x)|^{p-1}+|v(x)|^{p-1}\right) .
$$

Isto implica que

$$
\begin{aligned}
\left.|| x\right|^{\alpha} \frac{|u(x)+t v(x)|^{p}-|u(x)|^{p}}{t} \mid & =p|x|^{\alpha}\left|\vartheta_{x}\right|^{p-1}|v(x)| \\
& \leq 4^{p-1} p\left(|x|^{\alpha}|v(x)||u(x)|^{p-1}+|x|^{\alpha}|v(x)|^{p}\right) .
\end{aligned}
$$

Agora, tendo em vista a imersão continua $H_{0}^{1}(B) \hookrightarrow L^{p}(B)$ para todo $1 \leq p \leq 2^{*}$, 
tem-se que $|v| \in L^{p}(B),|u|^{p-1} \in L^{\frac{p}{p-1}}(B)$ e pela desigualdade de Hölder (Teorema A.2), resulta que $|v||u|^{p-1} \in L^{1}(B)$. Além disso, tendo em vista que $|x|^{\alpha} \in L^{\infty}(B)$, então $|x|^{\alpha}|v||u|^{p-1} \in L^{1}(B)$ e também $|x|^{\alpha}|v|^{p} \in L^{1}(B)$.

Note que das estimativas (B.4)-(B.5) tem-se que são satisfeita as condições do Teorema da Convergência Dominada de Lebesgue (ver Teorema A.8), então aplicando este teorema e tendo em vista a expressão $(\mathrm{B} .3)$ conclui-se que

$$
\lim _{t \rightarrow 0} \frac{1}{2} \int_{B}|x|^{\alpha}\left(\frac{|u+t v|^{p}-|u|^{p}}{t}\right) d x=\int_{B}|x|^{\alpha}|u|^{p-2} u v d x, \quad \forall u, v \in H_{0}^{1}(B) .
$$

Assim, I é Gâteaux-diferenciável $H_{0}^{1}(B)$ e

$$
I^{\prime}(u) v=\int_{B}|x|^{\alpha}|u|^{p-2} u v d x, \quad \forall u, v \in H_{0}^{1}(B)
$$

Também $L$ é Gâteaux-diferenciável $H_{0}^{1}(B)$. De fato, sejam $u, v \in H_{0}^{1}(B)$. Então

$$
\begin{aligned}
\lim _{t \rightarrow 0} \frac{L(u+t v)-L(u)}{t} & =\lim _{t \rightarrow 0} \frac{1}{2}\left(\frac{\|u+t v\|^{2}-\|u\|^{2}}{t}\right) \\
& =\frac{1}{2} \lim _{t \rightarrow 0} \frac{\langle u+t v, u+t v\rangle-\langle u, u\rangle}{t} \\
& =\frac{1}{2} \lim _{t \rightarrow 0}[2\langle u, v\rangle+t\langle v, v\rangle] \\
& =\langle u, v\rangle .
\end{aligned}
$$

Asim,

$$
L^{\prime}(u) v=\langle u, v\rangle=\int_{B} \nabla u \cdot \nabla v d x, \quad \forall u, v \in H_{0}^{1}(B) .
$$

Portanto $J: H_{0}^{1}(B) \rightarrow \mathbb{R}$ é Gâteaux-diferenciável em $H_{0}^{1}(B)$ e de (B.6) e (B.7), tem-se

$$
J^{\prime}(u) v=\int_{B} \nabla u \cdot \nabla v d x-\int_{B}|x|^{\alpha}|u|^{p-2} u v d x, \quad \forall u, v \in H_{0}^{1}(B) .
$$

O segundo passo é provar que a derivada de Gâteaux $J^{\prime}$ é contínua. Primeiro observe que $I^{\prime}$ é contínua. Para isto considere

$$
\begin{aligned}
& H_{0}^{1}(B) \stackrel{i}{\hookrightarrow} L^{p}(B) \stackrel{\mathcal{N}_{\alpha}}{\rightarrow} L^{\frac{p}{p-1}}(B) \quad \stackrel{i^{*}}{\hookrightarrow}\left[H_{0}^{1}(B)\right]^{*} \\
& u \quad \mapsto \quad u \quad \mapsto|x|^{\alpha}|u|^{p-2} u \mapsto|x|^{\alpha}|u|^{p-2} u
\end{aligned}
$$

onde $i: H_{0}^{1}(B) \hookrightarrow L^{p}(B)$ é a imersão de Sobolev de $H_{0}^{1}(B)$ em $L^{p}(B)$.

Afirmação: $I^{\prime}=i^{*} \circ \mathcal{N}_{\alpha} \circ i$. 
De fato, para quaisquer $u, v \in H_{0}^{1}(B)$

$$
\begin{aligned}
\left\langle i^{*} \circ \mathcal{N}_{\alpha} \circ i(u), v\right\rangle_{\left(H_{0}^{1}\right)^{*}, H_{0}^{1}} & =\left\langle\left(\mathcal{N}_{\alpha} \circ i\right)(u), i(v)\right\rangle_{L^{\frac{p}{p-1}, L^{p}}}=\int_{B}\left(\mathcal{N}_{\alpha} \circ i\right)(u) i(v) d x \\
& =\int_{B} \mathcal{N}_{\alpha}(u) v d x=\int_{B}|x|^{\alpha}|u|^{p-2} u v d x=\left\langle I^{\prime}(u, v)\right\rangle_{\left(H_{0}^{1}\right)^{*}, H_{0}^{1}}
\end{aligned}
$$

e portanto a afirmação está provada.

Desde que o operador linear $i$ é contínuo, pela observação anterior a este lema conclui-se que o operador $i^{*}$ é também contínuo. Por outro lado, note que o operador $\mathcal{N}_{\alpha}$ assim definido é um operador de Nemytskii e considerando $q=\frac{p}{p-1}, r=p-1$ e $b \equiv 0$ no Teorema A.29, tem-se que $\mathcal{N}_{\alpha}$ é contínuo. Portanto $I^{\prime}$ é continua.

Para provar que $L^{\prime}$ é continua, note que

$$
\begin{aligned}
& L^{\prime}: H_{0}^{1}(B) \rightarrow\left[H_{0}^{1}(B)\right]^{*} \\
& u \quad \mapsto \quad L^{\prime} u \quad: H_{0}^{1}(B) \rightarrow \mathbb{R} \\
& v \quad \mapsto\left\langle L^{\prime} u, v\right\rangle=\int_{B} \nabla u \cdot \nabla v=\langle u, v\rangle .
\end{aligned}
$$

Pelo teorema da Representação de Riesz $L^{\prime}$ é isomorfismo isométrico, assim $L^{\prime}$ é contínua. Portanto $J \in C^{1}\left(H_{0}^{1}(B), \mathbb{R}\right)$.

Lema B.8. Seja $p \in \mathbb{R}$ com $1<p<2_{\alpha}^{*}$. O funcional energia $J_{\mathrm{rad}}: H_{0, \mathrm{rad}}^{1}(B) \rightarrow \mathbb{R}$ associado ao problema (2.1), definido por

$$
J_{\mathrm{rad}}(u)=\frac{1}{2} \int_{B}|\nabla u|^{2} d x-\frac{1}{p} \int_{B}|x|^{\alpha}|u|^{p} d x, \quad u \in H_{0, \mathrm{rad}}^{1}(B)
$$

está bem definido. Além disso, $J_{\mathrm{rad}}^{\prime} \in C^{1}\left(H_{0, \mathrm{rad}}^{1}(B), \mathbb{R}\right)$ com

$$
J_{\text {rad }}^{\prime}(u) v=\int_{B} \nabla u \cdot \nabla v d x-\int_{B}|x|^{\alpha}|u|^{p-2} u v d x, \quad \forall u, v \in H_{0, \text { rad }}^{1}(B) .
$$

Demonstração. Fazendo,

$$
J_{\text {rad }}(u)=L(u)-I(u),
$$

onde $L(u)=\frac{1}{2}\|u\|^{2}$ e $I(u)=\frac{1}{p} \int_{B}|x|^{\alpha}|u|^{p} d x$. Para garantir a boa definição de $J_{\text {rad }}$ é suficiente mostrar que o funcional $I$ está bem definido. Note que isto é consequência imediata das imersões continua de Sobolev $H_{0}^{1}(B) \hookrightarrow L^{p}\left(|x|^{\alpha}, B\right)$ para $1 \leq p \leq 2_{\alpha}^{*}$.

Procedendo de forma similar como foi feito no Lema B.7 e fazendo observação na parte da estimativa (B.5), onde para funções em $H_{0, \text { rad }}^{1}(B)$ o lado direito da desigualdade ainda vai pertencer ao espaço $L^{1}(B)$. De fato, para $u, v \in H_{0 \text {,rad }}^{1}(B)$ pelas imersões 
de Sobolev tem-se $|v| \in L^{p}\left(B,|x|^{\alpha}\right)$ e $|u|^{p-1} \in L^{\frac{p}{p-1}}\left(B,|x|^{\alpha}\right)$. De isto também pode-se provar que $|x|^{\frac{\alpha}{p}}|v| \in L^{p}(B)$ e $|x|^{\frac{p-1}{p} \alpha}|u|^{p-1} \in L^{\frac{p}{p-1}}(B)$. Assim aplicando as desigualdades de Hölder (Teorema A.2), resulta $\left.|x||v \|| u\right|^{p-1} \in L^{1}(B)$ e finalmente usando o teorema da Convergência Dominada de Lebesgue (Teorema A.8 conclui-se que $J_{\text {rad }}$ é Gâteaux-diferenciável sobre $H_{0, \text { rad }}^{1}(B)$ e sua derivada de Gâteaux é dada

$$
J_{\mathrm{rad}}^{\prime}(u) v=\int_{B} \nabla u \cdot \nabla v d x-\int_{B}|x|^{\alpha}|u|^{p-2} u v d x, \quad \forall u, v \in H_{0, \mathrm{rad}}^{1}(B) .
$$

Resta provar que $J_{\text {rad }}^{\prime}$ é continua em $H_{0, \text { rad }}^{1}(B)$. Para isto, define-se

$$
\begin{aligned}
& H_{0, \mathrm{rad}}^{1}(B) \stackrel{i_{\alpha}}{\hookrightarrow} L^{p}\left(B,|x|^{\alpha}\right) \stackrel{\mathcal{N}}{\rightarrow} L^{\frac{p}{p-1}}\left(B,|x|^{\alpha}\right) \stackrel{i_{\alpha}^{*}}{\rightarrow}\left[H_{0, \mathrm{rad}}^{1}(B)\right]^{*} \\
& u \quad \mapsto \quad u \quad \mapsto \quad|u|^{p-2} u \quad \mapsto|u|^{p-2} u
\end{aligned}
$$

Afirmação: $I^{\prime}=i_{\alpha}^{*} \circ \mathcal{N} \circ i_{\alpha}$.

De fato, para quaisquer $u, v \in H_{0, \mathrm{rad}}^{1}(B)$

$$
\begin{aligned}
\left\langle i_{\alpha}^{*} \circ \mathcal{N} \circ i_{\alpha}(u), v\right\rangle_{\left(H_{0, \mathrm{rad}}^{1}\right)^{*}, H_{0, \mathrm{rad}}^{1}} & =\left\langle\left(\mathcal{N} \circ i_{\alpha}\right)(u), i_{\alpha}(v)\right\rangle_{L^{\frac{p}{p-1}}\left(B,|x|^{\alpha}\right), L^{p}\left(B,|x|^{\alpha}\right)} \\
& =\int_{B}\left(\mathcal{N} \circ i_{\alpha}\right)(u) i_{\alpha}(v)|x|^{\alpha} d x=\int_{B} \mathcal{N}(u) v|x|^{\alpha} d x \\
& =\int_{B}|u|^{p-2} u v|x|^{\alpha} d x=\left\langle I^{\prime} u, v\right\rangle_{\left(H_{0, \mathrm{rad}}^{1}\right)^{*}, H_{0, \mathrm{rad}}^{1},}
\end{aligned}
$$

e portanto a afirmação está provada.

Assim $I^{\prime}$ é contínuo, pois é uma composição de operadores contínuos. Da proposição anterior, tem-se que $L^{\prime}$ é continuo. Portanto, $J_{\text {rad }}^{\prime} \in C^{1}\left(H_{0, \text { rad }}^{1}(B), \mathbb{R}\right)$. 


\section{Referências Bibliográficas}

[1] R. A. Adams, J. J. F. Fournier. Sobolev spaces. Second edition. Pure and applied mathematics. Elsevier, 2003.

[2] A. Ambrosetti, P. H. Rabinowitz. Dual variational methods in critical point theory and applications. J. Funct. Anal. 14 (1973), 349-381.

[3] M. Badiale, E. Serra. Multiplicity results for the supercritical Hénon equation. Adv. Nonlinear Stud. 4 (2004), 453-467.

[4] T. Bartsch, T. Weth, M. Willem. Partial symmetry of least energy nodal solutions to some variational problems. J. Anal. Math. 96 (2005), 1-18.

[5] E. Berchio, F. Gazzola, T. Weth. Radial symmetry of positive solutions to nonlinear polyharmonic Dirichlet problems. Journal für die reine und angewandte Mathematik, 620 (2008), 165-183.

[6] D. Bonheure, E. M. dos Santos, M. Ramos. Ground state and non-ground state solutions of some strongly coupled elliptic systems. Trans. Amer. Math. Soc. 364 (2012), no. $1,447-491$.

[7] D. Bonheure, E. M. dos Santos, M. Ramos. Symmetry and symmetry breaking for ground state solutions of some strongly coupled elliptic systems. J. Funct. Anal. 264 (2013), 62-96.

[8] H. Brezis. Functional analysis, Sobolev spaces and partial differential equations. Universitext, Springer, 2010.

[9] J. Byeon, Z.-Q. Wang. On the Hénon equation: asymptotic profile of ground states, I. Ann. I. H. Poincaré - AN 23 (2006), 803-828. 
[10] D. G. Costa. Tópicos em análise não linear e aplicações as equaçoes diferenciais. VIII Escola Latino-Americana de Matemática, IMPA, 1986.

[11] D. G. de Figueiredo. Lectures on the ekeland variational principle with applications and detours. Springer-Verlag, 1989.

[12] D. G. de Figueiredo, E. M. dos Santos, O. H. Miyagaki. Sobolev spaces of symmetric functions and applications. J. Funct. Anal. 261, (2011), no. 12, 3735-3770.

[13] D. G. de Figueiredo, P. L. LIONS, R. D. Nussbaum. A priori estimates and existence of positive solutions of semilinear elliptic equations. J. Math. pures et appl. 61, (1982), 41-63.

[14] E. M. dos Santos. On the existence of positive solutions for a nonhomogeneous elliptic system. Portugaliae Math. (N.S.), 66 (2009), no. 3, 347-371.

[15] L. C. Evans. Partial differential equations. Graduate Studies in Mathematics, 19, American Mathematical Society, Providence, RI, 1998.

[16] G. B. Folland. Introduction to partial differential equations. (2a Edição), Princeton University Press, 1995.

[17] B. Gidas, W.-M. Ni, L. Nirenberg. Symmetry and related properties via the maximum principle. Comm. Math. Phys. 68 (3) (1979), 209-243.

[18] D. Gilbarg, N. S. Trudinger. Elliptic partial differential equations of Second Order. Springer, 1998.

[19] M. Hénon. Numerical experiments on the stability of spherical stellar systems. Astronomy and astrophysics 24 (1973), 229-427.

[20] O. Kavian. Introduction à la théorie des points critiques: et applications aux problèmes elliptiques. Springer-Verlag, 1993.

[21] Y. Y. Li. Existence of many positive solutions of semilinear elliptic equations on annulus. J. Differential Equations 83 (1990), no. 2, 348-367.

[22] M. Montenegro. The construction of principal spectral curves for Lane-Emden systems and applications. Ann. Scuola Norm. Sup. Pisa Cl. Sci. (4) 29 (2000), no. 1, 193-229.

[23] W. M. Ni. A nonlinear Dirichlet problem on the unit ball and its applications. Indiana Univ. Math. J. 31 (1982), no. 6, 801-807. 
[24] F. Pacella. Symmetry results for solutions of semilinear elliptic equations with convex nonlinearities. J. Funct. Anal. 192 (1) (2002), 271-282.

[25] D. Smets, J. Su, M. Willem. Non radial ground state for the Hénon equation. Commun. Contemp. Math. 4 (2002), 467-480.

[26] D. Smets, M. Willem. Partial symmetry and asymptotic behavior for some elliptic variational problems. Calculus of Variations and Partial Differential Equations, Springer, 18 (2003), 57-75.

[27] M. Struwe. Variational methods. Applications to nonlinear partial differential equations and Hamiltonian systems. Fourth edition. A Series of Modern Surveys in Mathematics 34. Springer-Verlag, 2008.

[28] M. Willem. Minimax theorems. Progress in Nonlinear Differential Equations and their Applications 24. Birkhäuser, 1996. 